TESE DE DOUTORADO EM ENGENHARIA DE SISTEMAS ELETRÔNICOS E AUTOMAÇÃO

SIMULAÇÕES COMPUTACIONAIS DO ESCOAMENTO CARDIOVASCULAR GUIADAS POR RESSONÂNCIA MAGNÉTICA

Vinicius de Carvalho Rispoli

UNIVERSIDADE DE BRASÍLIA 
UNIVERSIDADE DE BRASÍLIA

FACULDADE DE TECNOLOGIA

DEPARTAMENTO DE ENGENHARIA ELÉTRICA

\title{
SIMULAÇÕES COMPUTACIONAIS DO ESCOAMENTO CARDIOVASCULAR GUIADAS POR RESSONÂNCIA MAGNÉTICA
}

\author{
Vinicius de Carvalho Rispoli
}

ORIENTADOR: João Luiz Azevedo de Carvalho

TESE DE DOUTORADO EM ENGENHARIA DE SISTEMAS ELETRÔNICOS E AUTOMAÇÃO

Publicação: PPGEA.TD 089/2014

Brasília/DF: Dezembro - 2014 
UNIVERSIDADE DE BRASÍLIA

Faculdade de Tecnologia

TESE DE DOUTORADO EM ENGENHARIA DE SISTEMAS ELETRÔNICOS E AUTOMAÇÃO

\section{SIMULAÇÕES COMPUTACIONAIS DO ESCOAMENTO CARDIOVASCULAR GUIADAS POR RESSONÂNCIA MAGNÉTICA}

\section{Vinicius de Carvalho Rispoli}

Tese de doutorado submetida ao Departamento de Engenharia Elétrica da Faculdade de Tecnologia da Universidade de Brasilia, como parte dos requisitos necessários para a obtenção do grau de doutor.

Banca Examinadora

Prof. João Luiz Azavedo de Carvalho, Ph.D.

$\mathrm{ENE} / \mathrm{UnB}$ (Orientador)

Profa. Mylène Christine Queiroz de Farias, Ph.D.

$\mathrm{ENE} / \mathrm{UnB}$ (Examinador Interno)

Prof. Manuel Nascimento Dias Barcelos Júnior, Ph.D.

$\mathrm{ENE} / \mathrm{UnB}$ (Examinador Interno)

Prof. Carlos Eduardo Rochitte, Dr.

InCor/SP - HC/SP - FM/USP (Examinador Externo)

Prof. Alberto Tannús, Dr.

IFSC/USP (Examinador Externo)

Prof. Eduardo Peixoto Fernandes da Silva, Ph.D.

ENE/UnB (Suplente) 


\section{FICHA CATALOGRÁFICA}

\begin{tabular}{|c|c|}
\hline \multicolumn{2}{|c|}{ Simulações Computacionais do Escoamento Cardiovascular } \\
\hline \multicolumn{2}{|c|}{ Guiadas por Ressonância Magnética. [Distrito Federal] 2014.} \\
\hline \multicolumn{2}{|c|}{ xviii, 111p., 210 x $297 \mathrm{~mm}$ (ENE/FT/UnB, Doutor, Tese de Doutorado). } \\
\hline \multicolumn{2}{|c|}{ Universidade de Brasília. Faculdade de Tecnologia. } \\
\hline \multicolumn{2}{|c|}{ Departamento de Engenharia Elétrica } \\
\hline 1. Ressonância Magnética & 2. Contraste de Fase \\
\hline 3. Codificação de Velocidade em Fourier & 4. Dinâmica dos Fluidos Computacional \\
\hline 5. Processamente de Imagens & 6. Filtragem \\
\hline I. $\mathrm{ENE} / \mathrm{FT} / \mathrm{UnB}$ & II. Título (série) \\
\hline
\end{tabular}

\section{REFERÊNCIA BIBLIOGRÁFICA}

RISPOLI, V. C. (2014). Simulações Computacionais do Escoamento Cardiovascular Guiadas por Ressonância Magnética. Tese de Doutorado em Engenharia de Sistemas Eletrônicos de Automação, Publicação PPGEA.TD-089/14, Departamento de Engenharia Elétrica, Universidade de Brasília, Brasília, DF, 111p.

\section{CESSÃO DE DIREITOS}

AUTOR: Vinicius de Carvalho Rispoli.

TÍTULO: Simulações Computacionais do Escoamento Cardiovascular Guiadas por Ressonância Magnética.

GRAU / ANO: Doutor / 2014

É concedida à Universidade de Brasília permissão para reproduzir cópias desta tese de doutorado e para emprestar ou vender tais cópias somente para propósitos acadêmicos e científicos. O autor reserva outros direitos de publicação e nenhuma parte dessa dissertação de mestrado pode ser reproduzida sem autorização por escrito do autor.

Vinicius de Carvalho Rispoli

QSC 05 Casa 11, Taguatinga Sul.

72.016-050 Brasília - DF - Brasil. 
Dedicatória

Esse trabalho é dedicado ao meu filho Miguel Morato de Oliveira Rispoli e à memória da minha mãe Rosislene de Carvalho Rispoli, que nos deixou durante a conclusão desse projeto.

Vinicius de Carvalho Rispoli 


\section{Agradecimentos}

Não sou muito bom com agradecimentos, mas começo com as quatro pessoas que foram fundamentais para a conclusão desse projeto. Na ausência de um deles esse trabalho não teria sido possivel. Primeiro, e mais importante, devo todos os agradecimentos ao meu orientador João Luiz Azevedo de Carvalho. Pelas conversas, ensinamentos, viagens, incentivos, parceria, paciência, risadas, enrolações (a maioria da parte dele) e algumas poucas broncas. Ele é um exemplo de orientador que deveria ser seguido no mundo acadêmico como um todo.

Em segundo lugar, gostaria de agradecer ao Jon-Fredrik Nielsen, meu orientador no estágio de doutorado. Toda a paciência que ele teve ao me ajudar com os experimentos do phantom, principalmente montando as sequências de pulsos e fazendo com que tudo desse certo no final.

Também não posso deixar de agradecer a ajuda do Diogo "Motoca" Costa, técnico de laboratório da FGA-UnB, que através da internet me deu toda o suporte possivel para que eu conseguisse montar o controlador do phantom e fizesse com que meus experimentos nos EUA fossem realizados com êxito.

Gostaria de agradecer ao Professor Krishna Nayak pela gentileza de nos ceder o phantom para realizarmos as medidas de contraste de fase que foram fundamentais para as análises desse trabalho.

Meus agradecimentos também a CAPES, FAP-DF, DPP/UnB e FINATEC pelos auxilios financeiros relacionados a esse trabalho. Eles permitiram a participação nos eventos internacionais, em que apresentei trabalhos relacionados a essa tese, e a minha ida a University of Michigan, para o Doutorado Sanduiche. Certamente essas viagens foram fundamentais para o término desse projeto e para o meu aprendizado.

Gostaria de agradecer também aos membros que participaram da banca, Dr. Carlos Rochitte, Dr. Alberto Tannús, Ph.D. Mylène Queiroz, Ph.D. Manuel Barcelos e o Ph.D. Eduardo Peixoto, pela cordialidade e pelos diversos comentários e sugestões que ajudaram a melhorar este trabalho e que, certamente, farão toda a diferença nas pesquisas futuras que esse projeto ainda irá proporcionar. 
Aos meus colegas e amigos da Faculdade UnB Gama gostaria também de prestar meus sinceros agradecimentos. Em algumas ocasiões relacionadas a este projeto, e também por outros motivos, em que precisei me ausentar ou precisei de alguma ajuda, vários dos meus colegas me ajudaram e estiveram por perto. São eles, Ricardo Fragelli, Adson Rocha, Lindomar Carvalho, Edson Alves, Alessandro Borges, Augusto Brasil, Hilmer Neri, Marcus Chaffim, Henrique Moura, Luciano Emídio e Edson Mintsu.

À minha mãe Rosislene de Carvalho Rispoli e aos meus irmãos Diogo e Glauber de Carvalho Rispoli que sempre estiveram ao meu lado, torceram por mim e de alguma forma, ou de outra, me ajudaram a realizar este trabalho. Aguentaram e aguentam o meu péssimo humor e o meu estresse constante. Também gostaria de agradecer as minhas cunhadas Carolina Girardi e Alaine Alves pelo suporte que deram a minha mãe, a mim e aos meus irmãos durante o período em que minha mãe esteve internada. Foi fundamental para que eu conseguisse fazer a minha qualificação a tempo e também para que eu conseguisse participar do congresso do ISMRM em Milão este ano.

Finalmente, gostaria de agradecer a Paloma Morato de Oliveira por todas as conversas, risadas, apoio e também pelas sucessivas e incansáveis brigas. De uma forma ou de outra você sempre me apoiou e me ajudou nesse processo, apesar de ter atrapalhado um bocado também. Seja estando ao meu lado, seja cuidando do Miguel, seja cuidando da minha mãe no hospital, ou seja me enlouquecendo como só você sabe fazer. Tudo isso foi importante para o resultado deste trabalho. Serei eternamente grato. Provavelmente, se você ler isto brigaremos de novo. Faz parte! 


\section{RESUMO}

As doenças cardiovasculares representam hoje uma das maiores causas de morte no mundo. Desta forma, o conhecimento dos padrões de escoamento sanguíneo no corpo humano se faz importante no diagnóstico e pesquisa de algumas doenças. Utilizando a ressonância magnética (RM), o escoamento sanguíneo pode ser mensurado in vivo diretamente usando ressonância magnética (RM) com contraste de fase $(\mathrm{CF})$ ou através da codificação de velocidade em Fourier (CVF). Por outro lado, o escoamento sanguíneo pode também ser simulado utilizando uma abordagem baseada em modelos da dinâmica dos fluidos computacional (DFC). O CF tem como desvantagens os longos tempos de aquisição, a resolução espaço-temporal limitada, efeitos de volume parcial e baixa relação sinal-ruído (SNR). Por outro lado, a codificação de velocidade em Fourier é um método de imageamento por ressonância magnética promissor para realizar medidas do escoamento cardiovascular. A CVF oferece SNR consideravelmente maior que o CF e também é robusto aos efeitos de volume parcial. Os conjuntos de dados com CVF são adquiridos com baixa resolução espacial devido aos longos tempos de aquisição relacionados a sua alta dimensionalidade, $(x, y, v, t)$, mas proporcionam a distribuição de velocidade associada a cada um dos seus grandes voxels. Entretanto, não um mapa de velocidades do escoamento. Finalmente, a DFC proporciona alta resolução temporal e espacial, além de tempos de aquisição reduzidos. Porém a sua acurácia é dependente das hipóteses do modelo. O objetivo deste trabalho é apresentar um método capaz de integrar medidas diretas de RM (CF ou CVF) a um algoritmo para determinar solução das equações da dinâmica dos fluidos, tendo como principal interesse a redução no tempo de exame num ambiente clínico. Desta forma, este trabalho apresenta duas contribuições originais: (i) um método para extrair mapas de velocidade de alta resolução espacial a partir de dados com CVF de baixa resolução espacial; e (ii) um método para reconstruir campos de velocidade que são influenciados ao mesmo tempo pelas medidas diretas por RM (CF ou CVF) e pelos modelos físicos da dinâmica dos fluidos. 


\begin{abstract}
Cardiovascular diseases represents today one of the leading causes of death worldwide. Thus, knowledge of the blood flow patterns in the human body is important for diagnosis and research of certain diseases. Using magnetic resonance (MRI), in vivo 3D blood flow patterns can be either measured directly using phase-contrast (PC) MRI or Fourier velocity encoding (FVE) MRI. On the other hand, blood flow patterns can be obtained from model-based computational fluid dynamics (CFD) calculations. PC-MRI suffers from long scan times, limited spatio-temporal resolution, partial-volume effects and low signal-to-noise ratio (SNR). Fourier velocity encoding (FVE) is a promising magnetic resonance imaging method for measurement of cardiovascular blood flow. FVE provides considerably higher SNR than phase contrast imaging, and is robust to partial-volume effects. FVE data is usually acquired with low spatial resolution, due to scantime restrictions associated with its higher dimensionality, $(x, y, v, t)$, and provides the velocity distribution associated with a large voxel, but does not directly provides a velocity map. Since the acquisition time is a disadvantage of FVE, then, preferably, it should be acquired with rapid spiral trajectories. Finally, CFD provides arbitrarily high spatial and temporal resolution and reduced scan times, but its accuracy hinges on the model assumptions. The objective of this work is to present a method capable of integrate direct MRI (PC or FVE) measures inside a CFD solver on the way to reduce scan time in a clinical environment. This work presents two main original contributions: (i) a method to derive velocity maps with high spatial resolution from low spatial resolution FVE data; and (ii) a numerical framework for constructing a flow field that is influenced by both direct measurements (PC-MRI or FVE) and a fluid physics model.
\end{abstract}




\section{SUMÁRIO}

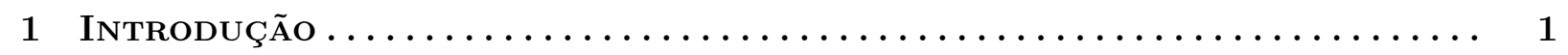

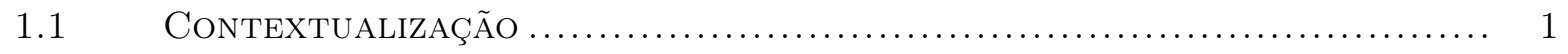



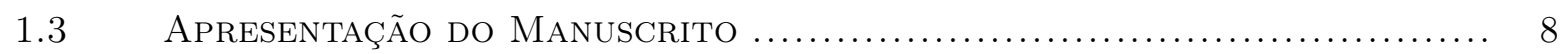

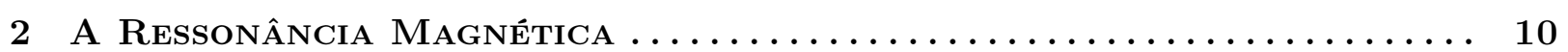

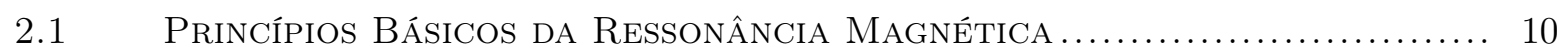

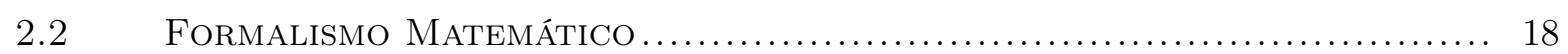

2.3 PRINCÍPIOS DO IMAGEAMENTO DE FluXo EM RESSONÂNCIA MAGNÉTICA. .... 19

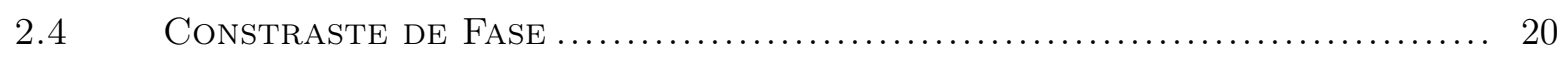

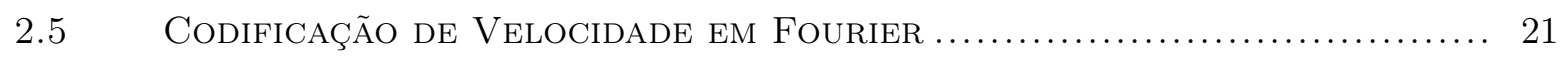

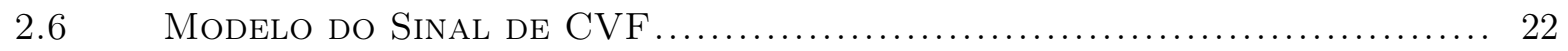

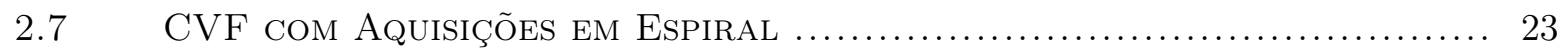

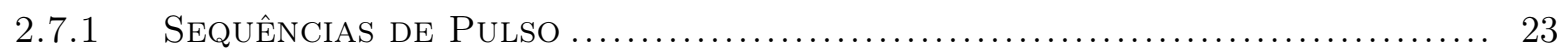

2.7 .2 MOdelo DE SinAL Do CVF COM AQUisições EM ESPIRAL .................... 23

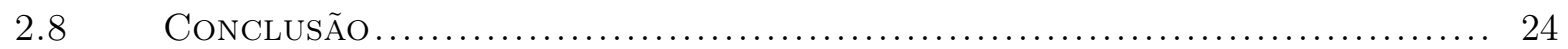

3 DinÂMICA DOS FlUIDOS COMPUTACIONAL.$\ldots \ldots \ldots \ldots \ldots \ldots \ldots \ldots \ldots$

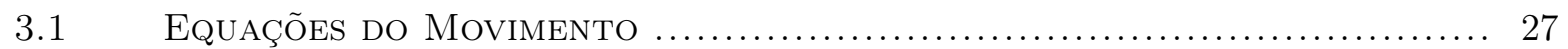

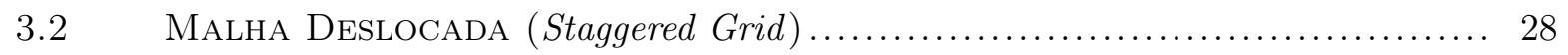

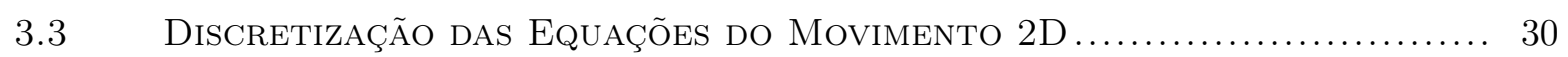

3.3 .1 EQUAÇÃO DO MOMENTUM NA DiReÇ̃̃̃O $x$..................................... 31

3.3 .2 EQUAÇÃO DO MOMENTUM NA DiReÇ̃̃̃O $y$..................................... 34

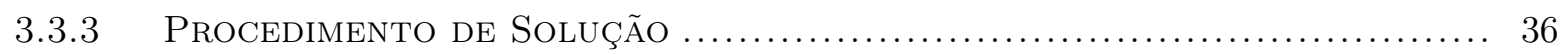



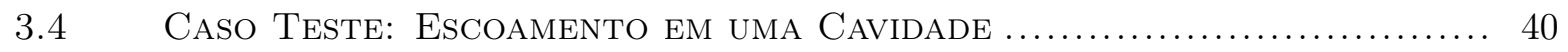


$3.5 \quad$ CONClUSÃO.

4 Simulações Computacionais do Escoamento Sanguíneo Guiadas Por

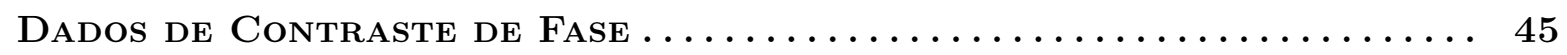

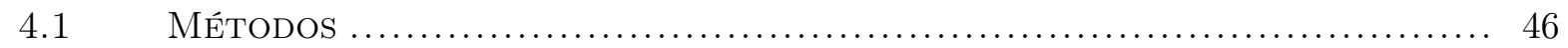

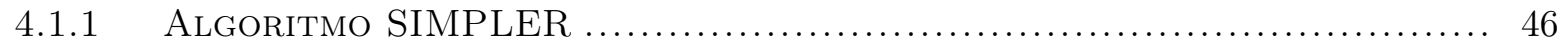

4.1 .2 INCORPORANDO AS MEDIDAS DE RM AO SIMPLER $\ldots \ldots \ldots \ldots \ldots \ldots \ldots \ldots \ldots \ldots$

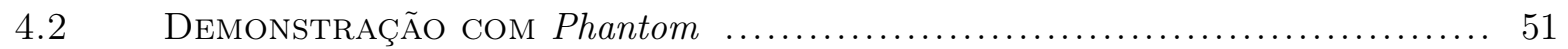

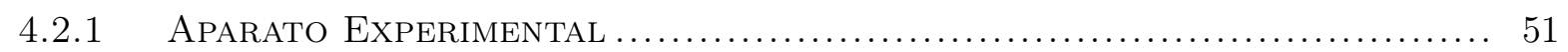

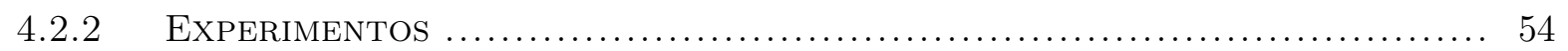

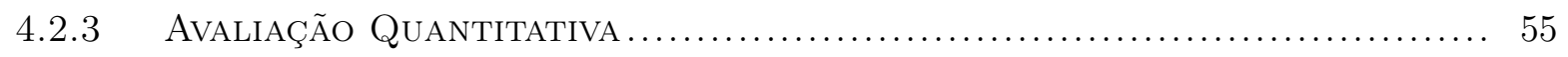

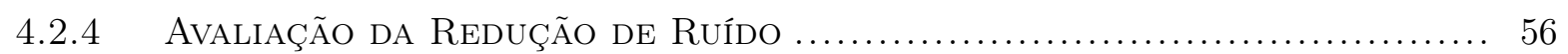

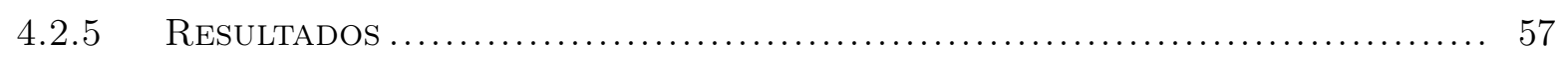

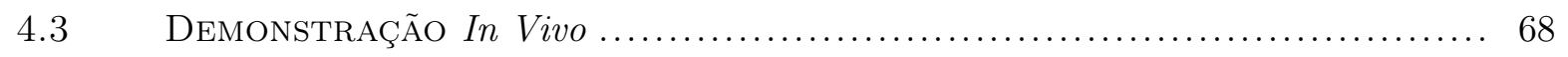

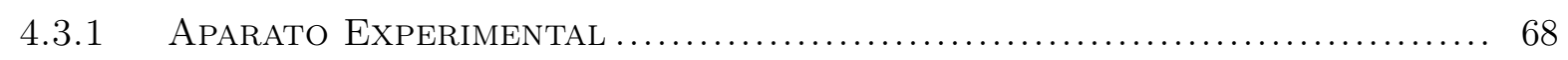

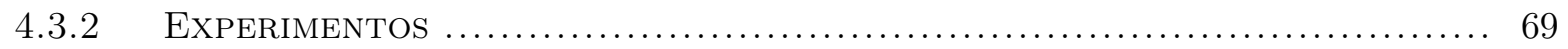

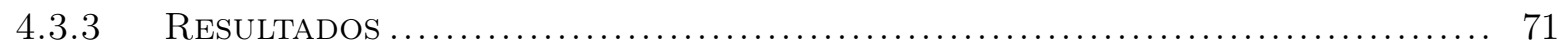

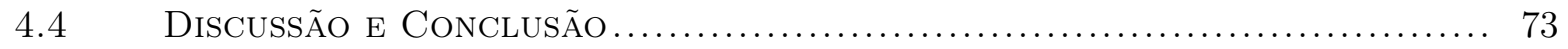

5 Simulações Computacionais de Esconmento SANGuíneo Guiadas Por

DAdos de RM COM CODIFICAÇÃo de Velocidade EM FouRIER . . . . . . . 81

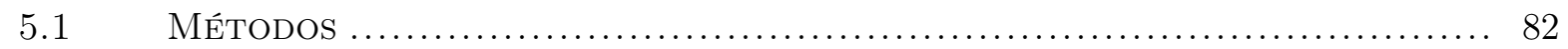

$5.1 .1 \quad$ Algoritmo DE DECONVOLUÇÃO NÃO-CEGA $\ldots \ldots \ldots \ldots \ldots \ldots \ldots \ldots \ldots \ldots \ldots \ldots$

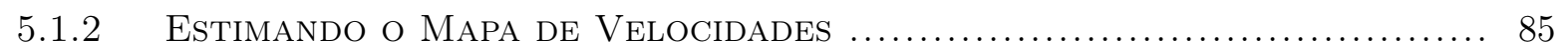

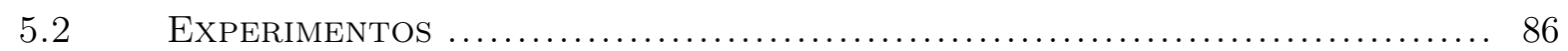

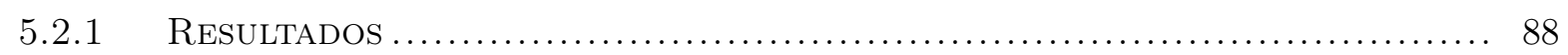

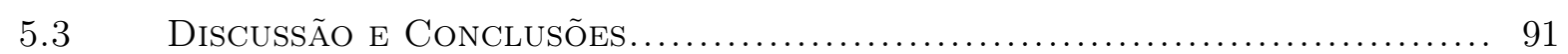

6 Conclusões e Trabalhos Futuros $\ldots \ldots \ldots \ldots \ldots \ldots \ldots \ldots \ldots \ldots \ldots$

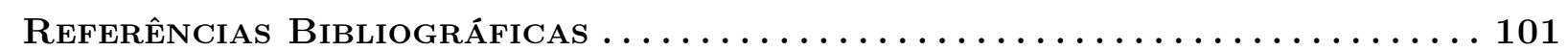

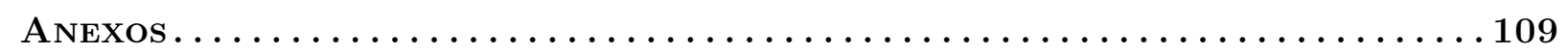

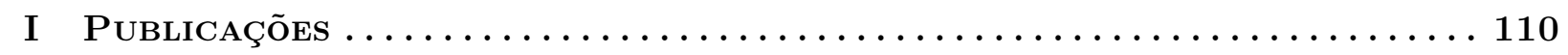




\section{LISTA DE FIGURAS}

1.1 Exemplo de aplicação da dinâmica dos fluidos computacional na engenharia biomédica. 4

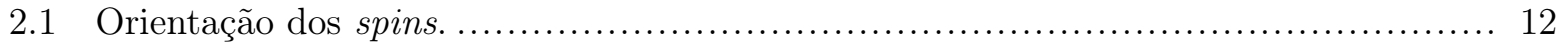

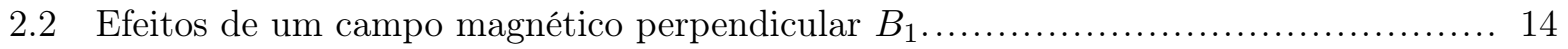

2.3 Efeitos da aplicação de um pulso $B_{1}$ de $180^{\circ}$ após um pulso de $90^{\circ} . \ldots \ldots \ldots \ldots \ldots \ldots \ldots$

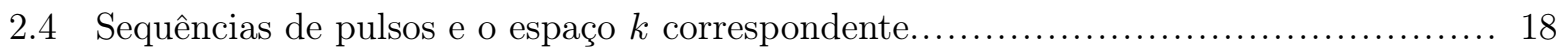

$2.5 \quad$ Princípios da codificação de velocidade em RM. . ..................................... 20

$2.6 \quad$ Sequência de pulsos da CVF em espiral. ........................................ 24

$2.7 \quad$ Esquema de amostragem do espaço $k$ para o CVF em espiral. ........................... 25



3.2 Volume de controle para a equação da continuidade. ................................... 31

3.3 Volume de controle para a equação do momentum na direção $x$. . ....................... 33

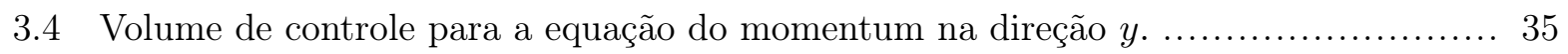

$3.5 \quad$ Ilustração do problema do escoamento em uma cavidade. ............................. 41

$3.6 \quad$ Solução numérica para o escoamento em cavidade. ..................................... 43

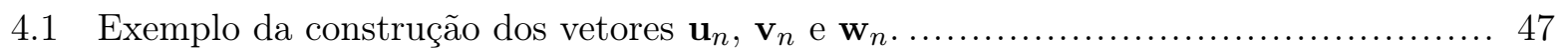

4.2 Componentes do phantom de escoamento pulsátil. .................................. 53

$4.3 \quad$ Esquema do controlador do phantom. ........................................... 54

4.4 Seções transversais da componente de velocidade na direção $u$ do phantom em quatro instantes de tempo diferentes para os diferentes tipos de estimação. ..................... 61

4.5 Seções transversais da componente de velocidade na direção $v$ do phantom em quatro instantes de tempo diferentes para os diferentes tipos de estimação. ................. 62

4.6 Seções transversais da componente de velocidade na direção $w$ do phantom em quatro instantes de tempo diferentes para os diferentes tipos de estimação. . . .

4.7 Seções transversais do $\nabla \cdot \vec{\nu}$ do phantom em quatro instantes de tempo diferentes para os diferentes tipos de estimação. 
4.8 Visualização do campo de velocidades do fluido do phantom para os diferentes tipos de estimação em quatro instantes de tempo diferentes.

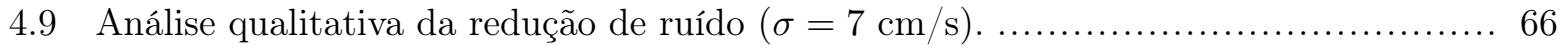

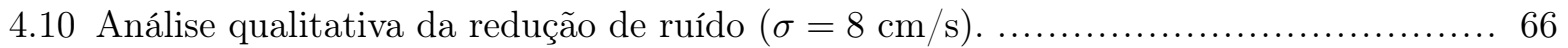

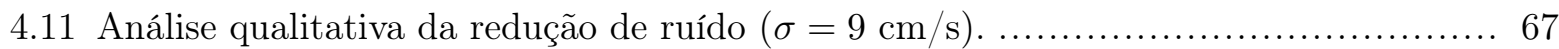

4.12 Seções transversais da componente de velocidade na direção $u$ em quatro instantes do ciclo cardíaco para os diferentes tipos de estimação.

4.13 Seções transversais da componente de velocidade na direção $v$ em quatro instantes do ciclo cardíaco para os diferentes tipos de estimação.

4.14 Seções transversais da componente de velocidade na direção $w$ em quatro instantes do ciclo cardíaco para os diferentes tipos de estimação. .

4.15 Seções transversais do $\nabla \cdot \vec{\nu}$ em quatro instantes do ciclo cardíaco para os diferentes tipos de estimação.

4.16 Visualização do escoamento do sangue na artéria carótida para os diferentes tipos de estimação em quatro instantes do ciclo cardíaco.

5.1 Avaliação qualitativa dos mapas de velocidade obtidos a partir de distribuições com eCVF.

5.2 Erro absoluto entre o mapa de velocidades medido por CF e os mapas de velocidade recuperados das distribuições com eCVF.

5.3 Seções transversais das componentes de velocidade e do erro para os diferentes tipos de estimação.

5.4 Visualização do campo de velocidades do phantom para os diferentes experimentos utilizando a eCVF. 


\section{LISTA DE TABELAS}

4.1 Relação sinal-erro entre o campo de velocidades medido por CF do phantom e os

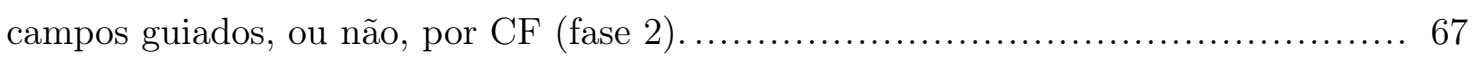

4.2 Relação sinal-erro entre o campo de velocidades medido por CF do phantom e os

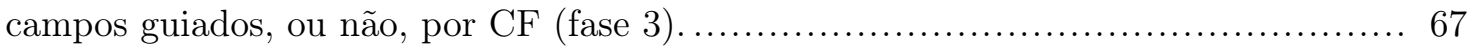

4.3 Relação sinal-erro entre o campo de velocidades medido por CF do phantom e os campos guiados, ou não, por CF (fase 4). ........................................ 68

4.4 Relação sinal-erro entre o campo de velocidades medido por CF do phantom e os

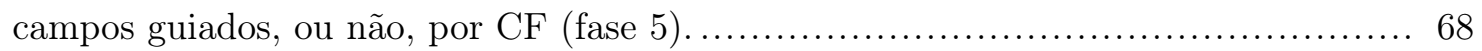

4.5 Análise quantitativa da redução de ruído utilizando o método proposto. ................ 68

4.6 Relação sinal-erro entre o campo de velocidades in vivo medido por CF e os campos

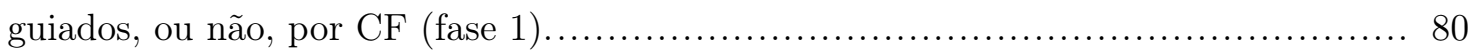

4.7 Relação sinal-erro entre o campo de velocidades in vivo medido por CF e os campos

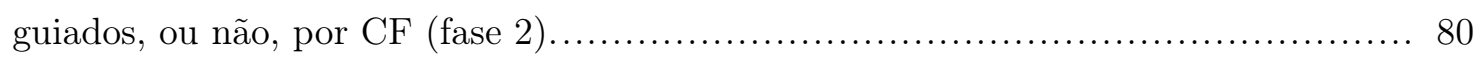

4.8 Relação sinal-erro entre o campo de velocidades in vivo medido por CF e os campos

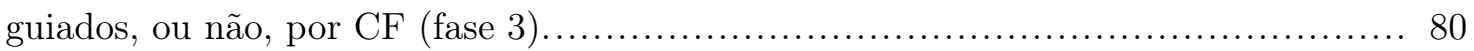

4.9 Relação sinal-erro entre o campo de velocidades in vivo medido por CF e os campos guiados, ou não, por CF (fase 4).

5.1 Relação sinal-erro e erro absoluto médio entre o mapa de velocidade medido por CF e os recuperados das distribuições de velocidade com eCVF.

5.2 Relação sinal-erro entre o campo de velocidade medido por CF e os campos de velocidade estimados com a DFC. . 


\section{LISTA DE SÍMBOLOS}

\section{Símbolos Latinos}

$\mathbf{A}_{u, n-1} \quad$ Matriz quadrada obtida na linearização das equações do movimento na direção $x$ para a $n$-ésima iteração

$\mathbf{A}_{v, n-1} \quad$ Matriz quadrada obtida na linearização das equações do movimento na direção $y$ para a $n$-ésima iteração

$\mathbf{A}_{w, n-1} \quad$ Matriz quadrada obtida na linearização das equações do movimento na direção $z$ para a $n$-ésima iteração

$\mathbf{A}_{u, \mathrm{cf}} \quad$ Matriz que representa as velocidades medidas por contraste de fase na malha da simulação computacional na direção $x$

$\mathbf{A}_{v, \mathrm{cf}} \quad$ Matriz que representa as velocidades medidas por contraste de fase na malha da simulação computacional na direção y

$\mathbf{A}_{w, \mathrm{cf}} \quad$ Matriz que representa as velocidades medidas por contraste de fase na malha da simulação computacional na direção $w$

$\mathbf{b}_{u, n-1} \quad$ Vetor obtido na linearização das equações do movimento na direção $x$ para a $n$-ésima iteração

$\mathbf{b}_{v, n-1} \quad$ Vetor obtido na linearização das equações do movimento na direção $y$ para a $n$-ésima iteração

$\mathbf{b}_{w, n-1} \quad$ Vetor obtido na linearização das equações do movimento na direção $z$ para a $n$-ésima iteração

$B_{0} \quad$ Campo magnético principal da máquina de ressonância magnética

$B_{1} \quad$ Campo magnético associado ao pulso de rádiofrequência

$G_{x} \quad$ Intensidade do gradiente na direção $x$

$G_{y} \quad$ Intensidade do gradiente na direção $y$

$G_{z} \quad$ Intensidade do gradiente na direção $z$ 
Coordenada de Fourier na direção $x$

Coordenada de Fourier na direção $y$

Coordenada de Fourier na direção z

Variável de Fourier $\left(k_{x}, k_{y}, k_{z}\right)$ associado ao vetor posição $\vec{r}$

Coordenada de Fourier da velocidade na direção $x$

Coordenada de Fourier da velocidade na direção $y$

$[\mathrm{s} / \mathrm{m}]$

Coordenada de Fourier da velocidade na direção $z$

$[\mathrm{s} / \mathrm{m}]$

Variável de Fourier $\left(k_{u}, k_{v}, k_{w}\right)$ associado ao vetor velocidade $\vec{\nu}$

Pressão hidrodinâmica

Vetor posição $(x, y, z)$

Tempo de retorno da magnetização para o eixo longitudinal

Tempo de redução da magnetização no plano transversal contraste de fase na direção $x$

Componente escalar do campo de velocidade simulado pela

dinâmica dos fluidos computacional na direção $x$ na $n$-ésima iteração

Componente escalar do campo de velocidade simulado pela dinâmica dos fluidos computacional na direção $x$ no estado estacionário

Componente escalar do campo de velocidade na direção y contraste de fase na direção $y$

Componente escalar do campo de velocidade simulado pela dinâmica dos fluidos computacional na direção y na $n$-ésima iteração

Componente escalar do campo de velocidade simulado pela dinâmica dos fluidos computacional na direção $y$ no estado estacionário 


\begin{tabular}{|c|c|}
\hline$w$ & Componente escalar do campo de velocidade na direção $z$ \\
\hline$w_{\mathrm{cf}}$ & $\begin{array}{l}\text { Componente escalar do campo de velocidade quantificado por } \\
\text { contraste de fase na direção } z\end{array}$ \\
\hline$w_{n}$ & $\begin{array}{l}\text { Componente escalar do campo de velocidade simulado pela } \\
\text { dinâmica dos fluidos computacional na direção } z \text { na } n \text {-ésima } \\
\text { iteração }\end{array}$ \\
\hline$w_{\infty}$ & $\begin{array}{l}\text { Componente escalar do campo de velocidade simulado pela } \\
\text { dinâmica dos fluidos computacional na direção } z \text { no estado } \\
\text { estacionário }\end{array}$ \\
\hline $\bar{w}_{\text {cvf }}$ & $\begin{array}{l}\text { Componente escalar do campo de velocidade recuperado da } \\
\text { distribuição com eCVF na direção } z \text { utilizando a Eq. } 5.9\end{array}$ \\
\hline$\hat{w}_{\mathrm{cvf}}$ & $\begin{array}{l}\text { Componente escalar do campo de velocidade recuperado da } \\
\text { distribuição com eCVF na direção } z \text { utilizando a Eq. } 5.10\end{array}$ \\
\hline$s$ & $\begin{array}{l}\text { Modelo de sinal que incorpora os efeitos de truncamento do } \\
\text { espaço } k \text { de uma distribuição adquirida por CVF com baixa } \\
\text { resolução espacial }\end{array}$ \\
\hline$\tilde{s}$ & $\begin{array}{l}\text { Distribuição aproximada adquirida por CVF com alta resolu- } \\
\text { ção espacial }\end{array}$ \\
\hline$x$ & Posição espacial na direção i \\
\hline$y$ & Posição espacial na direção j \\
\hline$z$ & Posição espacial na direção $\mathbf{k}$ \\
\hline
\end{tabular}

\section{Símbolos Gregos}

$\begin{array}{ll}\delta x & \text { Resolução espacial na direção } x \\ \delta y & \text { Resolução espacial na direção } y \\ \delta z & \text { Resolução espacial na direção } z \\ \delta r & \text { Resolução espacial da distribuição por eCVF simulada } \\ \delta t & \text { Passo de tempo computacional } \\ \delta w & \text { Resolução de velocidade da distribuição por CVF simulada } \\ \gamma & \text { Razão giromagnética } \\ \phi & \text { Fase de magnetização } \\ \mu & \text { Viscosidade dinâmica } \\ \rho & \text { Densidade }\end{array}$




$\begin{array}{llr}\vec{\nu} & \text { Campo de velocidade vetorial } & {[\mathrm{m} / \mathrm{s}]} \\ \vec{\nu}_{\mathrm{cf}} & \text { Campo de velocidade vetorial quantificado por contraste de } & {[\mathrm{m} / \mathrm{s}]} \\ & \text { fase } & {[\mathrm{m} / \mathrm{s}]} \\ \vec{\nu}_{n} & \text { Campo de velocidade vetorial simulado pela dinâmica dos flui- } & \\ & \text { dos computacional na } n \text {-ésima iteração } & {[\mathrm{m} / \mathrm{s}]} \\ \vec{\nu}_{\infty} & \text { Campo de velocidade vetorial simulado pela dinâmica dos flui- } & \\ & \text { dos computacional no estado estacionário } & {[1 / \mathrm{s}]}\end{array}$

\section{Constantes Adimensionais}

Re Número de Reynolds

\section{Siglas}

CF Contraste de fase

CFV Codificação de velocidade em Fourier

DFC Dinâmica dos fluidos computacional

eCFV Codificação de velocidade em Fourier com aquisições em espiral

NEX Número de repetições

RF Rádiofrequência

RM Ressonância magnética

SER Relação sinal-erro

SIL Sinal de indução livre

SNR Relação sinal-ruído

TE Tempo de eco

TF2D Transformada de Fourier bidimensional (amostragem cartesiana)

TF3D Transformada de Fourier tridimensional (amostragem cartesiana)

TR Tempo de repetição

VENC Codificação de velocidade 


\section{Capítulo 1}

\section{Introdução}

\subsection{Contextualização}

Segundo dados do Ministério da Saúde, as doenças cardiovasculares são a principal causa de morte em mulheres e homens no Brasil. Elas são responsáveis por cerca de 30\% de todas as mortes em indivíduos acima de 30 anos [1, 2]. Algumas dessas doenças são causadas ou podem ser diagnosticadas pelo escoamento anormal do sangue em uma determinada parte do sistema cardiovascular. Por exemplo, a aterosclerose consiste no estreitamento de um vaso sanguíneo devido ao acumulo gradual de lipídios, células inflámatórias e tecido conjuntivo na parede do vaso [3]. Esse estreitamento altera o escoamento sanguíneo local, podendo causar jatos de fluxo e/ou escoamentos turbulentos. Nos jatos ocorrem picos de velocidade significativamente maiores que aqueles apresentados em um escoamento normal. Dessa forma, o conhecimento dos padrões de escoamento sanguíneo no corpo humano é um componente importante na pesquisa e diagnóstico de certas doenças cardiovasculares. Atualmente, duas abordagens distintas para o estudo e quantificação do escoamento sanguíneo no corpo humano estão disponíveis aos pesquisadores e clínicos: (i) medidas diretas in vivo do campo de velocidades usando ressonância magnética (RM) com codificação de velocidade ou ultrassonografia com Doppler (US), por exemplo; e (ii) também a simulação do escoamento sanguíneo baseada em modelos físicos por meio de métodos de dinâmica dos fluidos computacional.

A ultrassonografia com Doppler é, em geral, o método mais utilizado para a quantificação dos padrões de escoamento sanguíneo no ambiente clínico. Seu equipamento é 
relativamente pequeno, barato e portátil, e é capaz de produzir medidas em tempo real com excelente resolução temporal. No exame, o feixe do ultrassom precisa ser alinhado corretamente com o eixo do escoamento pelo operador do equipamento, caso contrário as medidas podem não ser precisas. Além disso, se no caminho do feixe de ultrassom há cicatrizes cirúrgicas, gordura, osso ou ar, a quantificação do escoamento também pode ser comprometida.

A ressonância magnética é capaz de visualizar tridimensionalmente todos os aspectos de um exame cardíaco, como anatomia do coração, características no vasos sanguíneos e também a quantificação de velocidade em um determinado vaso. Comparada à ultrassonografia, a ressonância magnética não possui a mesma depêndencia do operador, sendo capaz de quantificar com precisão a direção correta do escoamento, e também não possui as mesmas limitações acústicas relacionadas a ossos, gordura, ar ou cicatrizes cirúrgicas. O método mais utilizado na quantificação do escoamento por RM é a técnica de constraste de fase $(\mathrm{CF})$ [4, 5, 6, 7, 8, 9, 10, 11, 12, 13. Nesta técnica, um gradiente bipolar é alinhado ao eixo do escoamento para se obter uma medida de velocidade (aproximadamente a média [14]) para cada voxel — elemento de volume tridimensional — da imagem. Apesar da sua utilização irrestrita, o contraste de fase apresenta algumas limitações. Uma das mais notáveis é a subestimação da velocidade devido aos efeitos de volume parcial [15]. Isso ocorre quando se tem uma larga distribuição de velocidades dentro de um voxel. Esse tipo de situação pode ser contornada aumentando a resolução espacial, o que resulta em uma diminuição drástica da relação sinal-ruído. Isto ocorre devido a diminuição da quantidade de spins dentro do voxel, o que leva a uma diminuição do módulo do vetor magnetização resultante. Dessa forma, uma das características do contraste de fase é que essa técnica oferece resoluções espaciais e temporais limitadas, o que, inevitavelmente, impacta na precisão das medidas. Além disso, aquisições por contraste de fase temporalmente resolvidas demandam longos tempos de aquisição (chegando a dezenas de minutos), que podem limitar o seu uso clínico.

A codificação de velocidade em Fourier (CVF) [16, 17, 18, 19, 20, 21, 22, 23, 24, 25] é uma técnica de codificação de velocidade em ressonância magnética que se apresenta como uma alternativa ao contraste de fase. Nessa técnica, as medidas obtidas possuem relação sinal-ruído consideravelmente maiores que as do contraste de fase, devido ao seu conjunto de dados de alta dimensão e também aos seus voxels maiores. Além disso, a CVF não sofre 
dos efeitos de volume parcial do CF, pois para cada voxel é medida sua distribuição de velocidades. O conjunto de dados medido com CVF normalmente é obtido com resolução espacial muito baixa. Isso se deve a restrições associadas a sua alta dimensionalidade, que pode acarretar em um longo tempo de aquisição. Dessa forma, a CVF não é uma técnica popular no ambiente clínico. Por outro lado, já foi mostrado que a aquisição de dados usando a CVF com trajetórias rápidas em espiral, no espaço $k$, é uma alternativa confiável para medir com precisão e acurácia picos de velocidade em jatos ou para se obter parâmetros hemodinâmicos do escoamento de forma rápida e confiável [24].

Através das medidas de velocidade adquiridas com ambas as técnicas de codificação de velocidade é possível obter parâmetros hemodinâmicos (tais como tensão de cisalhamento na parede ou gradientes de pressão) (Fig. 1.1) que possuem relação com a progressão e com o diagnósticos de algumas doenças. O cálculo desses parâmetros são áreas ativas e promissoras de pesquisa [26, 27, 28]. Enquanto esses biomarcadores, baseados nas imagens de ressonância magnética, podem proporcionar medidas hemodinâmicas relevantes, a habilidade da ressonância magnética de proporcionar informações absolutas relativas a esses parâmetros ainda é um problema em aberto. A falta de medidas absolutas torna difícil a comparação dos resultados obtidos através de tipos de esquemas distintos de aquisição e reconstrução (por exemplo, trajetórias Cartesianas ou espirais no espaço $k$ ). Isso pode limitar a pesquisa dos mecanismos biológicos responsáveis pela formação de ateromas nas paredes das artérias, por exemplo.

Com relação aos métodos indiretos de quantificação do escoamento, temos a dinâmica dos fluidos computacional (DFC) [29, 6, 30, 31, 32, 33, 34, 35, 36, 37, 38, 39, 40, 41] que é uma alternativa que vem sendo vastamente utilizada para prever os padrões de escoamento. Há estudos em diversas geometrias vasculares, incluindo aneurismas intracranianos [41, a aorta torácica [40] e a bifurcação carotídea, tanto em modelos [31, 34, 36, 38] quanto in vivo [32]. Na dinâmica dos fluidos computacional convencional, a anatomia tridimensional do lumén é especificada, juntamente com os perfis de velocidade na entrada e saída da artéria no domínio de cálculo. As equações que descrevem o movimento de um fluido são, então, solucionadas numericamente. Essa abordagem proporciona resoluções espaciais e temporais arbitrárias, e são, em princípio, capazes de estimar escoamentos em diversos vasos sanguíneos de geometrias complexas. Além disso, a dinâmica dos fluidos computacional necessita de um menor tempo de examinação dos pacientes, comparada ao contraste de 


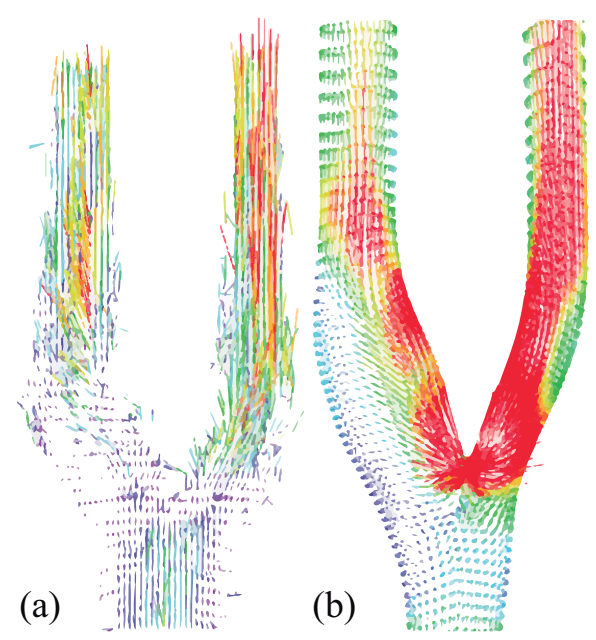

Figura 1.1: Exemplo de aplicação da dinâmica dos fluidos computacional na engenharia biomédica. Vetores da tensão de cisalhamento na parede na bifurcação carotídea: (a) estimado diretamente de ressonância magnética por contraste de fase; e (b) estimado pela dinâmica dos fluidos computacional (modificado da Ref. [34]).

fase, pois apenas uma imagem volumétrica é necessária (para determinação da geometria) e as velocidades de entrada e saída nos vasos sanguíneos podem ser obtidas com apenas duas aquisições rápidas por contraste de fase.

A precisão das rotinas convencionais da dinâmica dos fluidos computacional apresenta limitações com relação às suas diversas hipóteses de modelagem. Algumas hipóteses utilizadas na literatura não são necessariamente realistas para escoamentos vasculares in vivo, como vasos sanguíneos com paredes rígidas e viscosidade uniforme do sangue. Inclusive, a escolha apropriada do modelo físico a ser utilizado ainda é uma questão em aberto [41]. Os campos de velocidade simulados com a dinâmica dos fluidos computacional não possuem uma concordância unânime com as medidas por contraste de fase [34, 38, 40, 41], e a aplicabilidade da dinâmica dos fluidos computacional para estimação robusta do escoamento ainda está sendo debatida. Além disso, as rotinas comerciais, e mais utilizadas, da dinâmica dos fluidos computacional normalmente empregam malhas não Cartesianas de elementos finitos, para descrever melhor a geometria dos vasos sanguíneos, o que leva a algoritmos altamente complexos, implicando em um custo computacional muito elevado.

Algumas abordagens com o intuito de integrar as duas técnicas, isto é, construir campos de velocidade que são ao mesmo tempo influenciados pelas medidas reais in vivo e as simuladas pela dinâmica dos fluidos computacional já foram feitas. Um algoritmo de mínimos quadrados utilizando algumas considerações da mecânica dos fluidos (por exemplo, 
que uma linha de corrente não pode atravessar a parede do vaso sanguíneo) foi proposto para melhorar a qualidade das linhas de corrente para os dados medidos por contraste de fase [42. Também já foram propostos diversos métodos com o intuito de reduzir ruídos nos dados do contraste de fase levando em consideração que os campos de velocidades devem satisfazer a equação da continuidade [43, 44, 45]. Além disso, outros métodos de aquisições de velocidades in vivo já foram usados em simulações de dinâmica dos fluidos computacional, como no caso da Ref. [46], que introduziu as velocidades do sangue medidas com a ultrassonografia como um termo de força dentro das equações do movimento.

No entanto, grande parte da pesquisa científica considera as medidas obtidas com a ressonância magnética e a dinâmica dos fluidos computacional como abordagens mutuamente distintas e excludentes, isto é, o escoamento no interior do domínio tridimensional de cálculo é tipicamente ditado ou pelas medidas diretas ou modeladas pela dinâmica dos fluidos, e não pelas duas ao mesmo tempo. Em outras palavras, mesmo que se tenham disponíveis medidas do contraste de fase de uma ou mais componentes de velocidade no interior do domínio de cálculo, não há nenhuma maneira capaz de usar esses dados para influenciar a solução da dinâmica dos fluidos computacional. Assim, o pesquisador precisa, portanto, fazer uma decisão binária entre quais das duas abordagens usará para uma dada aplicação. Além disso, não existe um critério definitivo para a escolha de qual deverá ser usado.

\subsection{Objetivos do Trabalho}

O objetivo deste trabalho é realizar simulações computacionais de escoamentos sanguíneos guiadas por imagens de ressonância magnética com quantificação de fluxo. Tais simulações são realizadas a partir da discretização do sistema tridimensional de equações de Navier-Stokes e da equação da continuidade [47]:

$$
\begin{aligned}
\rho\left(\frac{\partial \vec{\nu}}{\partial t}+\vec{\nu} \cdot \nabla \vec{\nu}\right) & =-\nabla p+\mu \Delta \vec{\nu} \quad \mathrm{e} \\
\nabla \cdot \vec{\nu} & =0
\end{aligned}
$$

que geram quatro sistemas lineares na forma:

$$
\mathbf{A}_{\omega} \boldsymbol{\omega}=\mathbf{b}_{\omega}
$$


em que $t$ é o tempo, $\rho$ é a densidade do fluido, $\mu$ é a viscosidade do fluido, $\vec{\nu}$ é o vetor velocidade e $\omega$ representa uma das quatro variáveis que desejamos obter: as velocidades escalares $u, v$ e $w$; e a pressão $p$. Esses sistemas são, então, modificados para incluir as medidas adquiridas utilizando técnicas da ressonância magnética a fim de obter soluções que se assemelham com as medidas reais e, ao mesmo tempo, sofram influência do modelo físico.

Desta forma, este trabalho apresenta três contribuições originais principais. Inicialmente, apresentamos uma versão que incorpora as três dimensões de velocidade medidas por contraste de fase no algoritmo proposto por Nielsen \& Nayak [48, 49], produzindo resultados qualitativamente e quantitativemente melhores e com menor custo computacional [50, 51]. Em seguida, propusemos um método para derivar mapas de velocidade a partir de distribuições de velocidade medidas com codificação de velocidade em Fourier [52, 53], pois, as distribuições com CVF não produzem diretamente os mapas de velocidade do escoamento medido. E, finalmente, foram realizadas simulações utilizando a dinâmica dos fluidos computacional sendo guiadas por distribuições de velocidade com codificação de velocidade em Fourier, baseado no método proposto de obtenção de mapas de velocidade.

O método proposto em [48, 49] por Nielsen \& Nayak difere das demais encontradas na literatura por permitir a incorporação de medidas realizadas por contraste de fase dentro dos cálculos da solução numérica, pois a mesma não utiliza a ressonância apenas como dado de entrada e saída para os cálculos computacionais, ou para reconstruir a geometria das artérias. Assim, a solução numérica calculada é uma versão da imagem de contraste de fase que satisfaz as equações da mecânica dos fluidos. No entanto, no método proposto pelos pesquisadores supracitados, é utilizada apenas uma dimensão de velocidade medida por CF para realizar a simulação numérica. Isso gera resultados que diferem significativamente do CF original, principalmente quando se analisa os resultados utilizando, na solução numérica, as três dimensões de velocidade, como foi observado na presente pesquisa [50, 51].

A possibilidade de incluir dentro da solução computacional dados de velocidade adquiridos por técnicas de ressonância magnética é de fundamental interesse na redução do tempo de exame de um paciente. De forma geral, o imageamento de fluxo por ressonância magnética é demasiadamente lento e produz normalmente um conjunto de dados com muito ruído em baixa resolução espacial. Ambas as técnicas utilizadas para o imageamento de fluxo, contraste de fase e codificação de velocidade em Fourier, possuem as suas vantagens e 
desvantagens. E, desta forma, é parte dos objetivos deste trabalho possibilitar a redução do tempo de aquisição desses dados utilizando modelos da dinâmica dos fluidos computacional que incorporam uma ou outra dessas técnicas de imageamento.

Por exemplo, os dados imageados com contraste de fase no ambiente clínico são normalmente adquiridas com voxels grandes e com poucas repetições (NEX), devido seu alto tempo de aquisição. Isso resulta em campos de velocidades com imprecisões e degradados por ruído. O método proposto neste trabalho pode ser utilizado, nesse caso, para reduzir o ruído e também melhorar a precisão dos campos de velocidade a partir de um determinado modelo físico da mecânica dos fluidos. Poderia ser utilizada para reduzir o tempo de exame, adquirindo a imagem com uma resolução espacial ainda pior e utilizando o método proposto para obter as velocidades em uma malha mais refinada. Por outro lado, as distribuições de velocidade adquiridas usando a codificação de velocidade em Fourier com aquisições em espiral (eCVF) são, normalmente, adquiridas de forma rápida e com baixa resolução espacial. Elas não possuem os mesmos problemas de relação sinal-ruído e efeitos de volume parcial que o contraste de fase, devido a sua alta dimensionalidade e também por fornecer para cada voxel a distribuição de velocidades e não apenas um único valor de velocidade como o CF. Os resultados que serão apresentados neste trabalho mostram que é possível reconstruir mapas de velocidade, semelhantes aos do contraste de fase, a partir de distribuições de velocidade com CVF e, desta forma, esses dados podem ser utilizados para guiar as simulações computacionais [52, 53]. Isto é, com aquisições rápidas de medidas de velocidade utilizando a ressonância magnética será possível produzir campos de velocidade influenciados pelas duas técnicas com tempo de aquisição clinicamente viável, favorecendo, novamente, a diminuição do tempo de exame de um paciente.

Observamos que o método apresentado neste trabalho é uma técnica de pósprocessamento das imagens de fluxo obtidas com a ressonância magnética e tem como finalidades: obter campos de velocidade próximos aos reais (medidos com as distintas técnicas de imageamento de fluxo por RM) que também satisfazem as equações da mecânica dos fluidos; reduzir o ruído nas medidas de velocidade; e, no ambiente clínico, ser usado para diminuir o tempo de exame. A escolha do modelo físico utilizado e do algoritmo da dinâmica dos fluidos computacional foi feita pensando na facilidade de implementação e na velocidade de convergência. O modelo físico escolhido para este trabalho é justificado pela sua utilização de forma corriqueira na literatura [43, 32, 54, 34, 36, 46, 25]. Os resultados 
aqui apresentados sugerem que esse método pode ser utilizado no âmbito clínico de algumas maneiras com o intuito de reduzir o tempo de exame.

Finalmente, este trabalho se apresenta como uma prova de conceito do método combinado entre dados adquiridos com ressonância magnética e a dinâmica dos fluidos computacional. As simulações computacionais aqui foram feitas em malhas Cartesianas com hipóteses não realistas acerca da modelagem do escoamento sanguíneo, como paredes rígidas e modelo Newtoniano para a viscosidade, utilizando o método dos volumes finitos. No entanto, além de ser uma modelagem comum na literatura, é possível torná-la mais realista utilizando uma modelagem com malhas mais gerais, levando em consideração paredes elásticas para os vasos e, também, um modelo não-Newtoniano para a viscosidade. O mesmo método apresentado neste trabalho pode ser implementado utilizando o método dos elementos finitos e/ou incorporando os efeitos mais realistas citados. No método dos elementos finitos as equações do movimento são discretizadas de forma diferente a realizada neste trabalho. No entanto, após a discretização por elementos finitos, o problema não-linear dado pelas Eqs. (1.1) e (1.2), novamente se transforma no problema de determinar a solução de sucessivos sistemas lineares na forma da Eq. (1.3). Conforme já explicitado, a modificação das matrizes $\mathbf{A}_{\omega}$ e dos vetores $\mathbf{b}_{\omega}$ são a base do método proposto neste trabalho e, portanto, poderia ser adaptada a outros algoritmos capazes de solucionar as equações do movimento. As grandes desvantagens da utilização do método dos elementos finitos estão relacionadas aos seus longos tempos de simulação e também a dificuldade de implementação.

\subsection{Apresentação do Manuscrito}

No Capítulo 2, será feita uma revisão bibliográfica sobre o imageamento por ressonância magnética. Serão descritos os princípios básicos da formação da imagem e também os princípios do imageamento do escoamento sanguíneo. Uma breve revisão sobre os principais métodos do imageamento de fluxo - contraste de fase e codificação de velocidade em Fourier — também será apresentada.

Um algoritmo capaz de determinar a solução numérica para as equações do movimento de um fluido em um domínio tridimensional será apresentado no Capítulo 3. Será feita a dedução do algoritmo SIMPLER [55] no seu caso bidimensional, apenas de forma didática. No entanto, foram implementadas duas versões do algoritmo: uma bidimensional, apenas 
para validação do funcionamento do algoritmo, e uma tridimensional, que será utilizada para realizar as simulações numéricas do escoamento sanguíneo guiado por dados de ressonânica magnética.

O método que utiliza aquisições de velocidades por contraste de fase para guiar as simulações computacionais será descrito no Capítulo 4. Serão apresentadas comparações entre as velocidades obtidas com a ressonância magnética e as soluções computacionais, guiadas pelas imagens de ressonância ou não. Resultados serão apresentados utilizando dados in vivo e de um phantom de escoamento pulsátil para guiar as simulações, mostrando que o método é capaz de corrigir o escoamento medido com a ressonância magnética de forma a satisfazer as equações da dinâmica dos fluidos e também reduzir o ruído. Um phantom é um objeto desenvolvido especialmente para ser imageado em um determinado equipamento médico. Em geral eles são utilizados para realizar testes diversos no sistema de imageamento como, por exemplo, testar sequências de pulso, melhores parâmetros de aquisição, bobinas, dentro outros.

Finalmente, no Capítulo 5 será apresentado um método capaz de obter mapas de velocidade (semelhantes ao contraste de fase) a partir de distribuições de velocidade adquiridas com CVF de baixa resolução espacial. Os resultados mostraram que é possível obter mapas de velocidade qualitativamente compatíveis com os dados de contraste de fase testados. Em seguida, utilizando os resultados apresentados, serão construídos campos de velocidade simulados pela DFC guiados pelos mapas de velocidade obtidos através de distribuições de velocidade com eCVF. A vantagem de se usar dados com eCVF é que eles possuem alta relação sinal-ruído e podem ser obtidos em tempo inferior aos dados de contraste de fase. Isso é interessante no ambiente clínico, tendo em vista que hoje exames relacionados ao sistema cardivascular demoram dezenas de minutos. As distribuições com eCVF utilizadas neste capítulo foram simuladas a partir dos dados de CF adquiridos com o phantom. 


\section{Capítulo 2}

\section{A Ressonância Magnética}

Neste capítulo, são discutidos brevemente os princípios da ressonância magnética, incluindo uma revisão sobre os métodos de quantificação do escoamento usando a ressonância magnética. As técnicas de constraste de fase, codificação de velocidade em Fourier e codificação de velocidade em Fourier com aquisições em espiral são discutidas aqui com um maior formalismo.

\subsection{Princípios Básicos da Ressonância Magnética}

A Ressonância Magnética (RM) é a única modalidade capaz de permitir avaliar todos os aspectos das doenças cardíacas. A RM é capaz de gerar imagens transversais em qualquer plano (incluindo planos oblíquos) e também de medir o fluxo sanguíneo. A aquisição das imagens é baseada no uso de campos magnéticos e ondas na faixa da radiofrequência. A RM não utiliza radiação ionizante, sendo, assim, altamente segura ao paciente.

O componente principal do equipamento de ressonância magnética é o seu campo magnético de grande intensidade e uniforme, chamado de campo $B_{0}$. A direção do campo magnético principal $B_{0}$ define a direção do eixo $z$, também chamada de sentido longitudinal. Nas máquinas de RM, em geral, esse campo magnético permanece sempre ligado, mesmo quando o equipamento não está sendo utilizado para nenhum exame. A RM é usada para fazer imagens dos núcleos de hidrogênio, por causa da sua abundância (cerca de 10\% do peso corporal) no corpo humano. Além disso, as características da RM se diferem bastante entre o hidrogênio presente no tecido normal e no tecido patológico. 
O átomo de hidrogênio, o mais simples da tabela periódica, possui como núcleo um próton. Os prótons são partículas carregadas positivamente, que possuem uma propriedade chamada spin ou momentum angular. Associado ao spin, o próton de hidrogênio possui outra propriedade chamada de momento magnético, que faz com que o mesmo se comporte como um pequeno imã, apresentando um campo magnético muito pequeno, emanando do pólo sul ao pólo norte. Em condições normais, cada núcleo aponta para uma direção aleatória, resultando em uma magnetização resultante nula (Fig. 2.1 a). Na presença de um campo magnético externo forte (como o campo $B_{0}$ ), os spins se alinharão na direção desse campo aplicado (Fig. 2.1p). No alinhamento dos spins com o campo magnético $B_{0}$, surge um movimento chamado de precessão. Esse movimento em torno do eixo $z$, o qual é ilustrado na Fig. 2.1 , lembra o de um pião rotacionando em torno de si mesmo (com eixo de rotação inclinado com relação ao chão) e precessando em torno do eixo $z$.

Sobre ação do campo magnético $B_{0}$, os spins não, todos, se alinham na mesma direção. Aproximadamente metade se alinha paralelamente e a outra metade anti-paralelamente ao campo. A distribuição dos spins em cada uma das direções é regida pela distribuição de Boltzmann:

$$
\frac{N_{p}}{N_{a p}}=\mathrm{e}^{-E / k T}
$$

em que $E$ é a diferença de energia entre os estados, $N_{p}$ é o número de spins alinhados paralelamente, $N_{a p}$ é o número de spins alinhados anti-paralelamente, $k$ é a constante de Boltzmann e $T$ é a temperatura. Para um campo magnético de 1,5 Tesla, na temperatura média do corpo humano, a diferença entre a quantidade de spins que se alinham paralelamente e anti-paralelamente é de aproximadamente cerca de cinco para um milhão [56]. Isto é suficiente para criar uma magnetização resultante $M_{0}$ não nula e capaz de produzir sinal detectável. A magnetização resultante é proporcional à intensidade do campo magnético $B_{0}$. Dessa forma, máquinas de ressonância magnética com campos magnéticos mais fortes (por exemplo, 3 Tesla) proporcionam uma relação sinal-ruído maior.

Outro aspecto importante dos equipamentos de ressonância magnética são as bobinas de gradiente. Elas são utilizadas para realizar a codificação espacial dos spins. Existem normalmente três bobinas de gradiente $\left(G_{x}, G_{y}\right.$ e $\left.G_{z}\right)$, que produzem uma perturbação intencional $\left(B_{z}\right)$ no campo magnético $B_{0}$ quando ligadas. Essas perturbações variam line- 


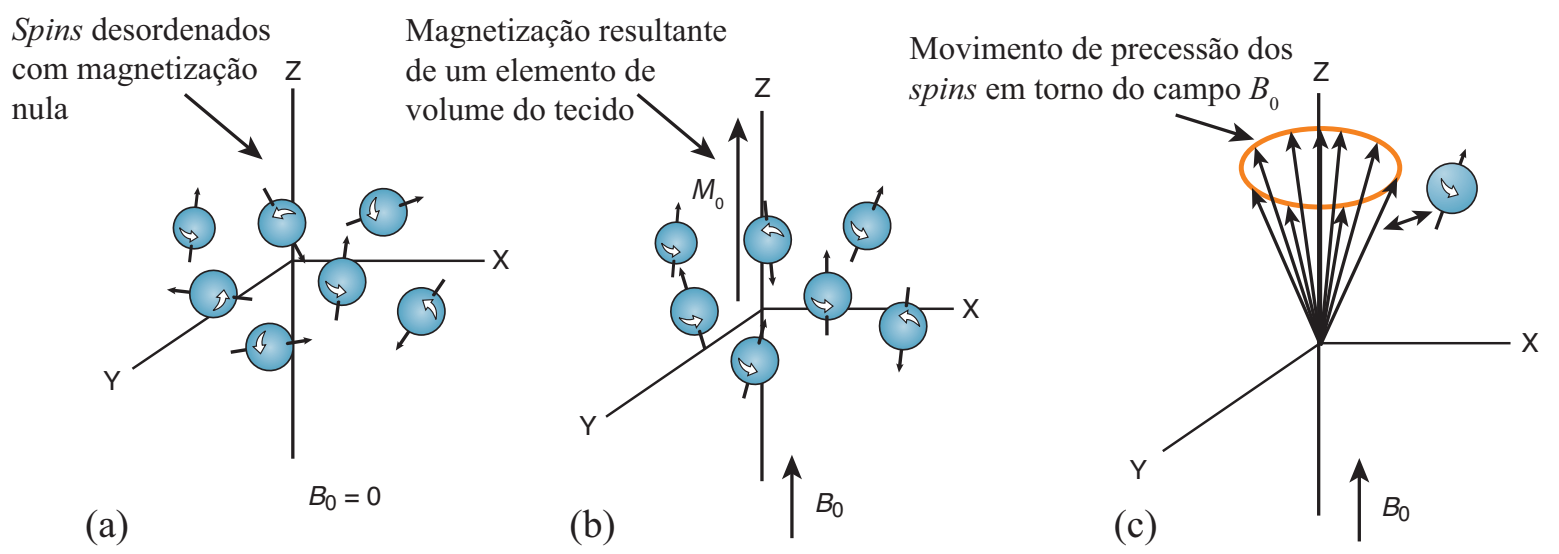

Figura 2.1: Orientação dos spins. (a) Sem ação do campo magnético, os spins são orientados aleatoreamente produzindo magnetização líquida nula. (b) Os spins se orientam com um campo magnético aplicado $\left(B_{0}\right)$, produzindo um campo magnético resultante alinhado na mesma direção de $B_{0}$. (c) Os spins magnetizados precessam em torno do campo $B_{0}$ (eixo $z$ ) de uma forma aleatória, não existe coêrencia de fase entre eles, resultando em uma magnetização nula no plano $x-y$ (modificado da Ref. [3]).

armente ao longo de cada direção espacial $(x, y$ e $z)$ :

$$
B_{z}(x, y, z)=B_{0}+x \cdot G_{x}+y \cdot G_{y}+z \cdot G_{z}
$$

em que $G_{x}, G_{y}$ e $G_{z}$ representam as intensidades do gradientes nas direções $x, y$ e $z$ e $B_{z}(x, y, z)$ é a intensidade do campo magnético perturbado, em função de sua posição espacial.

$\mathrm{Na}$ presença de um campo magnético externo, os spins precessam sobre o eixo do campo magnético (Fig. 2.1k). O campo $B_{0}$ é aproximadamente uniforme espacialmente, de forma que todos os spins precessam na mesma frequência (conhecida como frequência de Larmor),

$$
\omega=\gamma B_{0}
$$

em que $\gamma$ é a razão giromagnética ( $\gamma=42,58 \mathrm{MHz} /$ Tesla para prótons de hidrogênio). Entretanto, quando qualquer um dos gradientes é ligado, o campo magnético se torna variável no espaço, o que faz com que a frequência de precessão dos spins também o seja. O novo campo criado localmente com o acionamento do gradiente faz com que a frequência de precessão dos spins mude, ou seja, os spins em cada posição do tecido na direção de aplicação do gradiente precessa em uma frequência diferente. Dessa maneira, a frequência 
é utilizada, agora, para localizar espacialmente o sinal. O acionamento de um gradiente também altera a fase dos spins. Esta alteração é proporcional ao tempo que o gradiente fica ligado e a amplitude do gradiente. Juntas, fase e frequência fornecem informações espaciais do sinal. Portanto, os gradientes $G_{x}, G_{y}$ e $G_{z}$ são usados para codificar - em frequência e/ou fase - a posição espacial dos spins nas direções $x, y$ e $z$, respectivamente.

Para que uma corrente elétrica seja induzida na bobina de recepção, é necessário que o vetor magnetização $M_{0}$ como um todo, ou parte dele, esteja no plano transversal e possua coerência de fase. Se todos os momentos magnéticos individuais fossem desviados em $90^{\circ}$ para o plano transversal e todos estiverem em fase, teremos o máximo de sinal induzido na bobina. Para reorientar o vetor magnetização $M_{0}$, um segundo campo magnético de curta duração (pulso) e baixa intensidade precisa ser aplicado. Esse campo, denominado de $B_{1}$, deve ser perpendicular ao campo magnético principal $B_{0}$ e deve estar em ressonância com a frequência de precessão. Dessa forma, um terceiro componente importante da máquina de ressonância magnética é a bobina de radiofrequência $(\mathrm{RF})$. Ela é usada para transmitir uma excitação em RF para o corpo e, também, para receber o sinal codificado em frequência da porção excitada do corpo. Na prática, bobinas independentes podem ser usadas para a transmissão e a recepção. O pulso de RF é modulado para a frequência de Larmor, $\omega$. Como citado, enquanto $B_{0}$ está alinhado com a direção $z$ (por definição), $B_{1}$, que é um campo magnético muito fraco associado ao pulso de RF, estará alinhado ao eixo $x^{\prime}$ do referencial girante (também por definição). Quando o pulso de RF é ligado, alguns dos spins que estão em ressonância com o pulso de RF (i.e., precessando na frequência do pulso de RF) agora giram sobre o eixo y (por isso o nome ressonância magnética). Isto inclina a magnetização resultante sobre o plano $x-y$, que agora terá uma componente no plano $x-y$, $M_{x y}$ (Fig. 2.27).

O pulso de radiofrequência é normalmente desenhado para ter perfil aproximadamente retangular no domínio de Fourier, centrado na frequência de modulação (por exemplo, uma função sinc janelada e modulada). Isso implica que o pulso de RF na verdade contém uma certa quantidade de frequências, assim todos os spins rodando nesta faixa se tornam excitados, ou inclinados sobre o eixo y (Fig. 2.2 a). Desta forma, ligando gradiente(s) de uma amplitude apropriada, e planejando um pulso de RF de forma adequada, é possível excitar apenas uma fina fatia do corpo, que corresponde à região que contém todos os spins que estão em ressonância com a faixa de frequências do pulso de RF. Perfis de excitação 


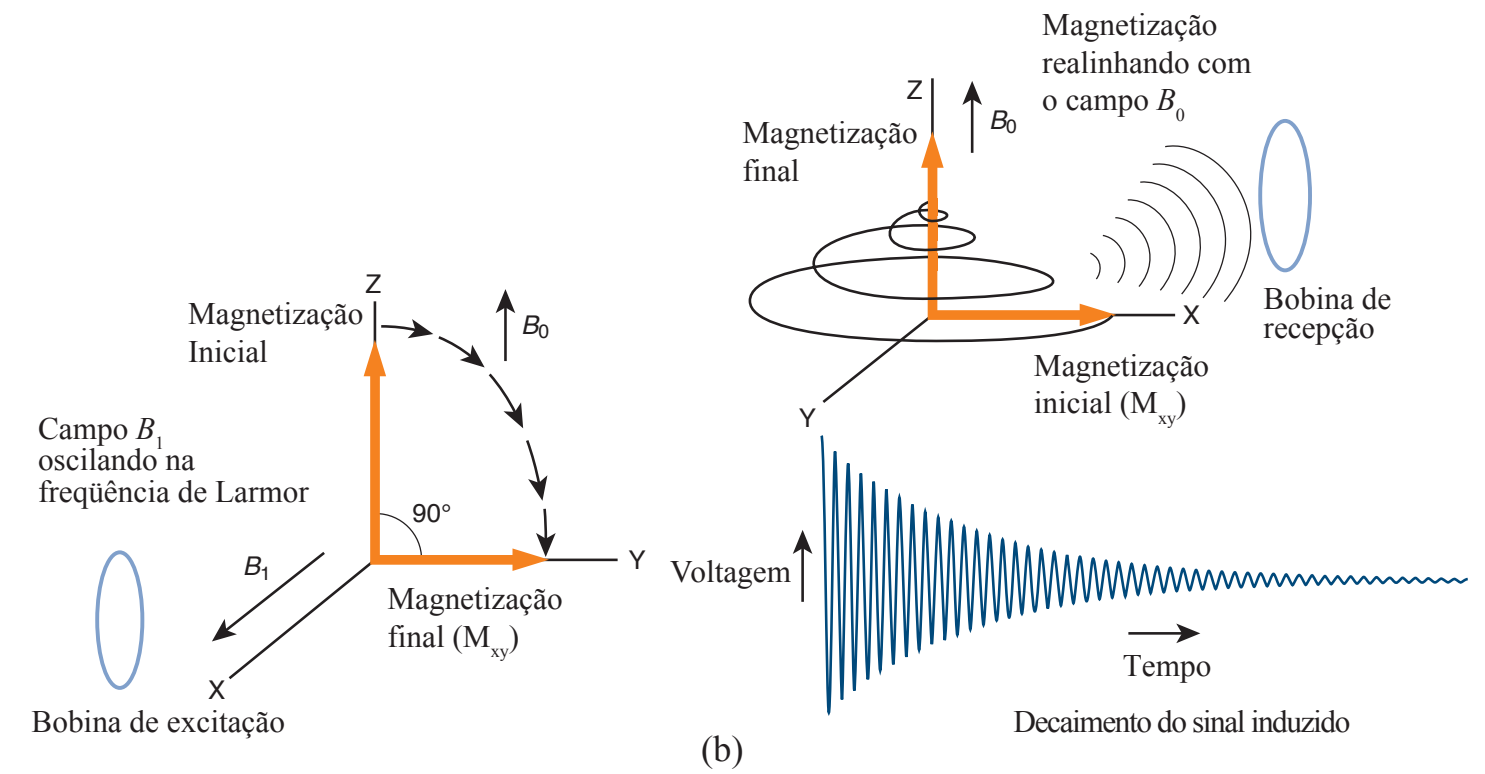

Figura 2.2: Efeitos de um campo magnético perpendicular $B_{1}$. (a) $\mathrm{O}$ campo $B_{1}$ oscilando na frequência de Larmor dos spins é absorvido pelos spins e causa uma rotação em torno ao campo $B_{0}$ (eixo $z$ ). (b) Uma vez que o campo $B_{1}$ é removido, os spins continuam a girar em torno do eixo $z$ na frequência de Larmor, mas o sinal decai (no plano $x-y$ ) enquanto se reestabelece o equilíbrio. O resultado é um campo magnético oscilatório, que é detectado com uma bobina enquanto o sinal decai. O sinal induzido é uma voltagem senoidal com frequência igual à frequência de Larmor dos spins (modificado da Ref. [3]).

diferentes podem ser obtidos também (por exemplo, uma excitação cilíndrica [57]), apenas planejando de forma apropriada a combinação gradiente-pulso.

Quando o pulso de radiofrequência é desligado, $M_{x y}$ continua girando (na frequência de Larmor) em torno do eixo $z$, enquanto a magnetização resultante começa a realinhar com o campo $B_{0}$. Essa magnetização girante gera um sinal oscilante, denominado de sinal de indução livre (SIL), que pode ser detectado pela bobina de recepção (Fig. 2.2b). A relaxação dos spins, após o desligamento do pulso de RF, que gera o SIL, é causada pelas trocas de energia entre spins e entre spins e sua vizinhança (rede). Essas interações são chamadas de relaxação spin-spin e spin-rede e, juntas, fazem com que o vetor $M_{x y}$ retorne ao seu estado de equilíbrio, i.e., paralelo ao campo $B_{0}$. Duas constantes de tempo foram criadas para caracterizar cada um desses processos: $T_{1}$ e $T_{2}$. A constante $T_{1}$ se relaciona com tempo de retorno da magnetização para o eixo longitudinal e é influenciada pela interação spin-rede. Por outro lado, a constante $T_{2}$ está relacionada à redução da magnetização no plano transversal e esta é influenciada pela interação spin-spin [56]. Após o pulso de RF, a magnetização na direção $z, M_{z}(t)$, é inicialmente nula, mas cresce com tempo de relaxação 
$T_{1}$ até o seu valor de equilíbrio, $M_{0}$ :

$$
M_{z}(t)=M_{0}\left(1-e^{-t / T_{1}}\right)
$$

em que $t$ é o tempo após o pulso de RF. Já a relaxação $T_{2}$ pode ser entendida como o tempo que os spins demoram para se defasarem no plano $x-y$. Após a excitação inicial usando o pulso $B_{1}$, todos os spins no plano $x-y$ possuem fases idênticas e, então, eles começam a defasagem rapidamente. Essa é descrita pela constante de tempo $T_{2}$. Ela leva a magnetização transversal a decair e se aproximar de zero devido a incoerência de fase. Levando em consideração a queda da magnetização, o sinal transversal, $M_{x y}(t)$, também decai de forma exponencial, com uma constante de tempo $T_{2}$ :

$$
M_{x y}(t)=M_{0} e^{-t / T_{2}}
$$

Importante ressaltar que cada tipo de tecido humano possui valores únicos para as constantes $T_{1}$ e $T_{2}$ [3]. Dessa forma, as diferenças entre os tempos de relaxação podem ser utilizadas para gerarem contrastes entre os diferentes tecidos presentes nas imagens. Em geral, dois processos distintos contribuem para a desfasagem dos spins. O primeiro mecanismo é a verdadeira interação spin-spin $\left(T_{2}\right)$, que é inevitável, irreversível e dependente das interações moleculares dentro da amostra. O segundo processo é chamado de decaimento $T_{2}^{*}$ (lê-se $T_{2}$ estrela) e inclui o relaxamento $T_{2}$ e também um relaxamento que é resultado da falta de homogeneidade do campo magnético principal $\left(B_{0}\right)$ dentro da amostra.

No tecido, o decaimento $T_{2}^{*}$ provoca uma relaxação magnética muito rápida dos prótons de hidrogênio em comparação com o relaxamento $T_{2}$. Assim, a taxa de decaimento do SIL é, na verdade, uma medida de $T_{2}^{*}$. Como $T_{2}^{*}$ é um processo mais rápido, ele limita o tempo de detecção do sinal de RM. No entanto, é possível contornar e reverter os efeitos da defasagem $T_{2}^{*}$. Depois da aplicação do pulso de $90^{\circ}$ (Fig. 2.3 a) naturalmente ocorre a defasagem dos spins (Fig. 2.3p). Dessa forma, outro campo $B_{1}$ é aplicado com o objetivo de girar a magnetização $180^{\circ}$ em torno do eixo $x$. Essa rotação de $180^{\circ}$ é conseguida através da aplicação do campo $B_{1}$ pelo dobro do tempo ou com o dobro da amplitude do pulso de $90^{\circ}$. Além disso, a rotação de $180^{\circ}$ faz com que os spins girem para o setor oposto do plano transversal. Deste lado do plano, os spins agora entram em fase novamente (Fig. 2.3.). Esta é a natureza do eco que recupera de forma eficaz toda a coerência de fase na magnetização 
(a)

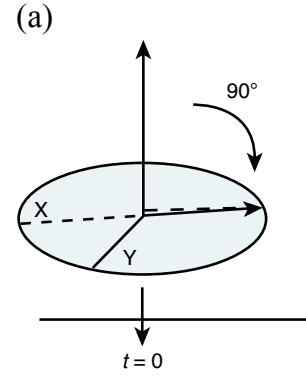

(b) Defasagem

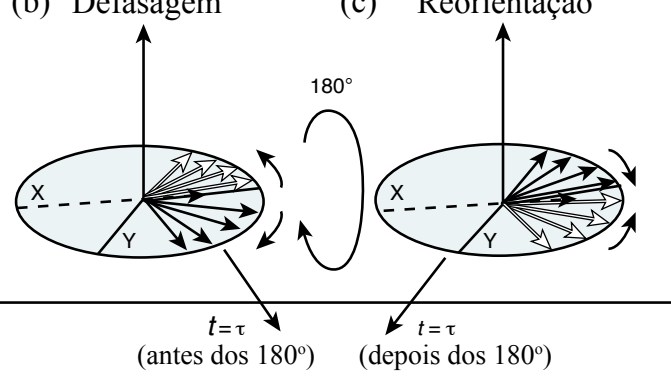

(c) Reorientação

(d) eco de spins

Tempo

Figura 2.3: Efeitos da aplicação de um pulso $B_{1}$ de $180^{\circ}$ após um pulso de $90^{\circ}$. (a) Os spins são inicalmente excitados por um pulso de $90^{\circ}$. (b) Depois de algum tempo em que ocorreu a defasagem dos spins, um pulso de a $180^{\circ}$ é aplicado. (c) O pulso de $180^{\circ}$ faz com que os spins girem $180^{\circ}$ em torno do eixo $x$. Isso troca a posição da fase dos spins mais lentos e dos mais rápidos, de modo que os spins mais rápidos possuam um atraso com relação aos spins mais lentos. Os spins mais rápidos começam a recuperar esse atraso, e depois de um tempo, $t$, igual ao tempo de defasagem, (d) os spins se alinham todos na mesma direção (ou seja, possuem a mesma fase). Este processo é chamado de reorientação e gera o que é chamado de eco de spins (modificado da Ref. [3]).

transversal, que havia sido destruído pela falta de homogeneidade campo $B_{0}$ na amostra (Fig. 2.3 d). O tempo entre o pulso inicial de RF de $90^{\circ}$ e o eco é um parâmetro importante na obtenção do sinal máximo detectável e é conhecido como tempo de eco (TE).

O conteúdo de frequência do sinal recebido é usado para obter a informação espacial sobre a porção excitada do corpo. Com o objetivo de codificar em frequência a posição espacial, gradientes são ligados durante a aquisição do sinal. Eles são chamados de gradientes de leitura. Para fazer a imagem de uma fatia perpendicular ao eixo $z$ (uma imagem axial), $G_{z}$ é ligado durante a excitação (para seleção da fatia), e $G_{x}$ e $G_{y}$ são ligados durante a aquisição. Esses podem ser trocados, para adquirir imagens sagitais ou coronais, ou todos os três gradientes podem ser usados juntos durante excitação e aquisição para obter imagens em planos oblíquos.

Quando os gradientes de leitura são ligados, o sinal adquirido em um instante particular de tempo corresponde a soma dos diferentes sinais senoidais gerados pelos spins localizados em diferentes regiões do corpo, cada um rodando a uma frequência diferente, correspondendo a sua posição espacial. Se uma fatia axial está sendo adquirida, por exemplo, o valor do sinal demodulado é equivalente a uma amostra da transformada de Fourier $M\left(k_{x}, k_{y}\right)$ da imagem transversal $m(x, y)$. Nesse caso, mudando as amplitudes dos gradientes $G_{x}$ e $G_{y}$ durante a aquisição, pode-se adquirir diferentes amostras de $M\left(k_{x}, k_{y}\right)$. Na 
verdade, ligando $G_{x}$ e/ou $G_{y}$, pode-se mover sobre o plano $k_{x}-k_{y}$ (que é conhecido como espaço $k)$, coletando amostras de $M\left(k_{x}, k_{y}\right)$. Quando uma quantidade suficiente de amostras de $M\left(k_{x}, k_{y}\right)$ foram coletadas a transformada inversa de Fourier produz a imagem $m(x, y)$.

A cobertura necessária do espaço $k$ e o número de amostras dependem da resolução espacial e do campo de visão desejados. Para imagens com baixa resolução espacial, apenas a porção central de $k_{x}-k_{y}$ precisa ser amostrada. Para uma resolução espacial maior, a periferia do espaço $k$ também precisa ser coberta. O campo de visão está associado com o espaçamento entre as amostras. Para um campo de visão grande, o espaço $k$ precisa ser densamente amostrado, requerendo uma grande quantidade de amostras. Se o espaço $k$ não é suficientemente amostrado, e, por isso, o campo de visão resultante não é suficientemente grande para cobrir o objeto por completo, sobreposições no domínio espacial serão observados (aliasing).

Como a amplitude do sinal decresce enquanto a magnetização resultante se realinha com $B_{0}$ (isto é chamado relaxação), múltiplas aquisições (excitação + leitura) podem ser necessárias para cobrir o espaço $k$ adequadamente. Alguns tipos de trajetórias são mais eficientes ao cobrir o espaço $k$ do que outras. Por exemplo, o imageamento espiral, que usa gradientes oscilatórios para gerar trajetórias espirais no espaço $k$ (Fig. 2.4b), são geralmente mais rápidas do que o imageamento Cartesiano, ou TF2D, i.e., necessita menos aquisições. No imageamento TF2D, cada leitura adquire uma única linha do espaço $k$, amostrando $k_{x}-k_{y}$ em uma forma cartesiana (Fig. 2.4 a). Isto é de forma geral uma aquisição mais lenta, mas que pode ser vantajosa para algumas aplicações com respeito a natureza dos artefatos associados a imagem. Existem outros tipos de estratégias para o preenchimento do espaço $k$ como imageamento eco planar, radial, cêntrico, dentre outras, e a escolha depende das necessidades do usuário [56].

Finalmente, para adquirir uma imagem de ressonância magnética, é necessário que uma combinação de pulsos de RF e gradientes sejam ligados de uma forma repetitiva durante um período de tempo. Assim, a forma em que os pulsos de RF e gradientes são ligados é chamado de sequência de pulsos. O tempo entre aquisições sucessivas é chamado de tempo de repetição (TR). 


\section{$\mathbf{a}$}

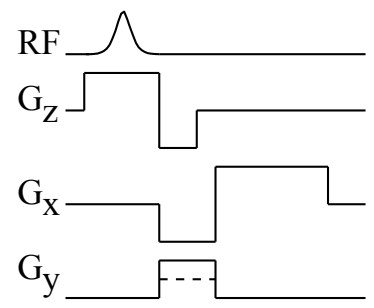

b
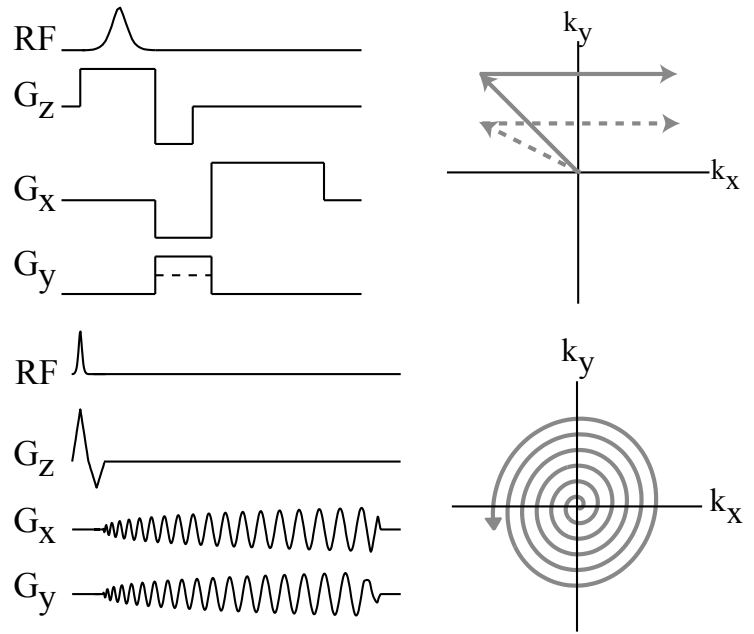

Figura 2.4: Sequências de pulsos (esquerda) e o espaço $k$ correspondente (direita) para (a) aquisições TF2D [58], e (b) aquisições em espiral.

\subsection{Formalismo Matemático}

Como discutido na seção anterior, o sinal de ressonância magnética demodulado $s(t)$ medido em um instante particular de tempo corresponde a uma amostra da transformada de Fourier $M\left(k_{x}, k_{y}\right)$ da imagem transversal $m(x, y)$ :

$$
M\left(k_{x}, k_{y}\right)=\int_{x} \int_{y} m(x, y) e^{-j 2 \pi\left(k_{x} x+k_{y} y\right)} d x d y .
$$

As coordenadas de Fourier $k_{x}$ e $k_{y}$ variam com o tempo, de acordo com os momentos de ordem zero dos gradientes de leitura $G_{x}$ e $G_{y}$ :

$$
\begin{aligned}
k_{x}(t) & =\frac{\gamma}{2 \pi} \int_{0}^{t} G_{x}(\tau) d \tau \\
k_{y}(t) & =\frac{\gamma}{2 \pi} \int_{0}^{t} G_{y}(\tau) d \tau .
\end{aligned}
$$

Essas equações explicam como os gradientes são usados para se mover ao longo do espaço $k$, como discutido na seção anterior. Esse formalismo pode ser generalizado para qualquer tipo de combinação dos gradientes $G_{x}, G_{y}$ e $G_{z}$ como:

$$
\begin{aligned}
M\left(\overrightarrow{k_{r}}\right) & =\int_{\vec{r}} m(\vec{r}) e^{-j 2 \pi k_{r} \cdot r} d \vec{r} \\
\overrightarrow{k_{r}}(t) & =\frac{\gamma}{2 \pi} \int_{0}^{t} \vec{G}_{r}(\tau) d \tau
\end{aligned}
$$


em que $\overrightarrow{G_{r}}=\left(G_{x}, G_{y}, G_{z}\right)$ é o gradiente oblíquo resultante da combinação dos gradientes $G_{x}, G_{y}$ e $G_{z}$ e $\vec{r}=(x, y, z)$ é a posição espacial.

Dada uma posição espacial temporalmente variável $\vec{r}(t)$ e um gradiente de campo magnético $\vec{G}_{r}(t)$, a fase de magnetização é:

$$
\phi(\vec{r}, t)=\gamma \int_{0}^{t} \vec{G}_{r}(\tau) \cdot \vec{r}(\tau) d \tau
$$

Para spins estáticos, $\vec{r}(t)$ é constante, e a Eq.2.11) acima fica:

$$
\begin{aligned}
\phi & =\gamma \vec{r} \cdot \int_{0}^{t} \overrightarrow{G_{r}}(\tau) d \tau \\
& =2 \pi \overrightarrow{k_{r}} \cdot \vec{r}
\end{aligned}
$$

como na exponencial na Eq. 2.9.

\subsection{Princípios do Imageamento de Fluxo em Ressonância Magné- tica}

Os princípios básicos da quantificação do escoamento usando a ressonância magnética foram propostos por Singer [59] e Hahn [60] no final dos anos 1950. Entretanto, aplicações clínicas do método proposto só foram relatados a partir do começo dos anos 1980 [61, 62]. A método utilizado para quantificação do escoamento usando ressonância magnética é baseado no fato que spins se movendo com uma velocidade $\vec{\nu}$ geram uma fase que é proporcional ao produto da velocidade com o primeiro momento do gradiente sobre a direção na qual os spins se movem (Fig. 2.5.

Isto é, para spins se movendo com velocidade constante $\vec{\nu}=(u, v, w)$, e posição inicial $\overrightarrow{r_{0}}$ podemos escrever a sua posição como $\vec{r}(t)=\vec{r}_{0}+\overrightarrow{\nu t}$. Reescrevendo a Eq. 2.11), para $t=T E:$

$$
\begin{aligned}
\phi & =\gamma \int_{0}^{T E} \overrightarrow{G_{r}}(t) \cdot\left(\vec{r}_{0}+\vec{\nu} t\right) d t \\
& =\gamma \overrightarrow{r_{0}} \cdot \int_{0}^{T E} \overrightarrow{G_{r}}(t) d t+\gamma \vec{\nu} \cdot \int_{0}^{T E} \overrightarrow{G_{r}}(t) t d t \\
& =\gamma \overrightarrow{r_{0}} \cdot \vec{M}_{0}+\gamma \vec{\nu} \cdot \vec{M}_{1} .
\end{aligned}
$$




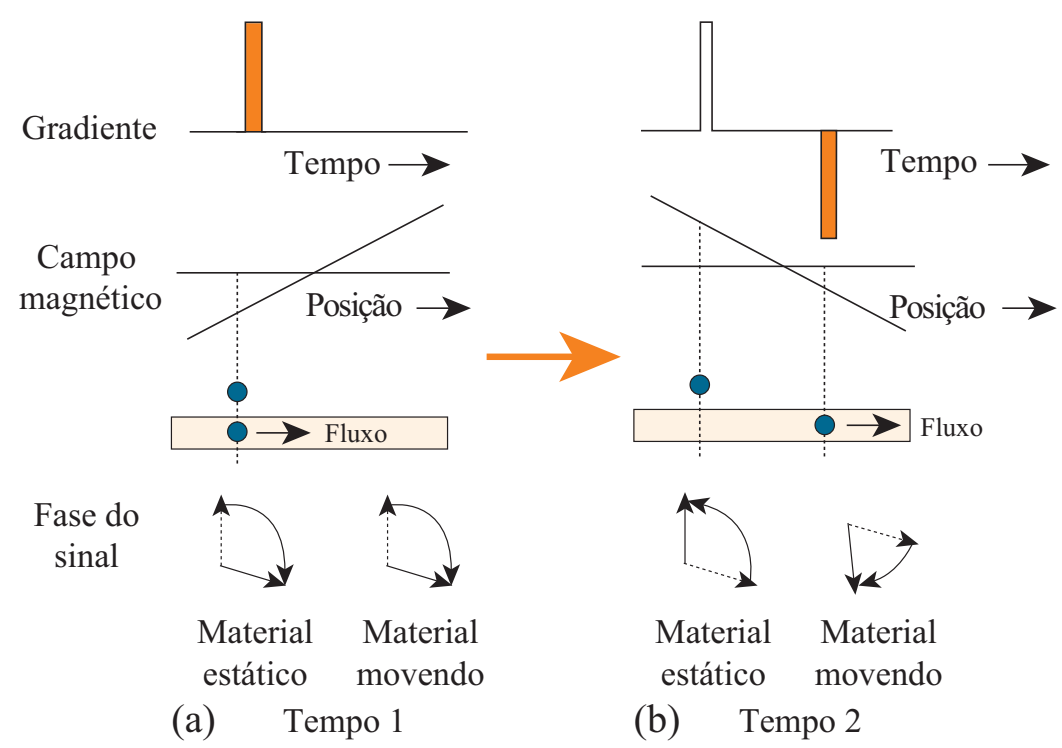

Figura 2.5: Princípios da codificação de velocidade em RM. (a) No tempo 1, um gradiente positivo é aplicado, o que resulta em uma frequência e uma mudança de fase associada igual para os spins vizinhos estacionários e em movimento, respectivamente. (b) No tempo 2, um gradiente de igual intensidade, mas de sinal oposto, é aplicado. Neste momento, os spins no sangue em movimento se separaram das suas vizinhanças originais e percebem uma intensidade diferente de campo magnético durante a aplicação do gradiente. O resultado é que, embora a fase dos spins estacionários será cancelada, a dos spins em movimento irá acumular uma mudança de fase proporcional à distância percorrida e, consequentemente, à velocidade (modificado da Ref. [3]).

em que $\vec{M}_{0}$ e $\vec{M}_{1}$ são os momento de ordem zero e primeiro momento do gradiente $\vec{r}$ no tempo de eco (TE), respectivamente. Assim, se um gradiente com momento de ordem zero nulo é usado (por exemplo, um gradiente bipolar alinhado com $\vec{\nu}$ ), a fase resultante para uma velocidade constante é $\phi=\gamma \vec{\nu} \cdot \vec{M}_{1}$.

Portanto, se um gradiente com forma de onda bipolar é usado entre as excitações e a leitura, a fase medida em um pixel da imagem adquirida é diretamente proporcional à velocidade dos spins contidos naquele respectivo voxel (Fig. 2.5). Entretanto, fatores além do escoamento (como não homonegeneidade do campo magnético) podem causar deslocamentos adicionais da fase, que podem levar a interpretações errôneas do campo de velocidade local 63.

\subsection{Constraste de Fase}

O método do contraste de fase soluciona o problema mencionado acima por utilizar duas aquisições em que o primeiro momento do gradiente bipolar é variado entre as medidas 
[4]. A velocidade em cada voxel ao longo de determinado eixo é medida como:

$$
w_{\mathrm{cf}}(x, y)=\frac{\phi_{a}(x, y)-\phi_{b}(x, y)}{\gamma\left(M_{1}^{a}-M_{1}^{b}\right)}
$$

em que $\phi_{a}(x, y)$ e $\phi_{b}(x, y)$ são as imagens de fase adquiridas em cada aquisição e $M_{1}^{a}$ e $M_{1}^{b}$ são os primeiros momentos dos gradientes bipolares usados nesse eixo nessas aquisições, respectivamente.

\subsection{Codificação de Velocidade em Fourier}

Enquanto o contraste de fase proporciona uma única medida de velocidade associado a cada voxel (aproximadamente a média [14]), a codificação de velocidade em Fourier (CVF) [16] fornece o histograma de velocidades para cada localização espacial, que é uma medida da distribuição de velocidade dentro de cada voxel.

A CVF envolve a codificação de fase da velocidade ao longo de uma dimensão de Fourier associada à velocidade. Ao contrário de apenas duas aquisições, como no contraste de fase, múltiplas aquisições são realizadas, e um gradiente bipolar com uma amplitude (e primeiro momento) diferente é usado em cada aquisição. A Eq. 2.13 pode ser reescrita como:

$$
\phi(\vec{r}, \vec{\nu}, t)=2 \pi\left(\overrightarrow{k_{r}} \cdot \vec{r}+\vec{k}_{\vec{\nu}} \cdot \vec{\nu}\right)
$$

em que $\vec{k}_{\vec{\nu}}=\left(k_{u}, k_{v}, k_{w}\right)$ é a variável de Fourier associada com $\vec{\nu}$, e é proporcional ao primeiro momento de $\vec{G}_{r}(t)$ :

$$
\vec{k}_{\vec{\nu}}=\frac{\gamma}{2 \pi} \vec{M}_{1}
$$

Supondo que há somente fluxo perpendicular ao plano $x-y$, isto é, $\vec{\nu}=(0,0, w)$, então cada voxel da imagem bidimensional está relacionado com uma distribuição de velocidade. A função tridimensional $m(x, y, w)$ está associada com o espaço de Fourier tridimensional $M\left(k_{x}, k_{y}, k_{w}\right)$. Desta forma, uma dimensão extra é adicionada ao espaço $k$ e múltiplas aquisições são necessáras para cobrir o espaço $k_{x}, k_{y}, k_{w}$. Com o intuito de se mover sobre $k_{w}$, um gradiente bipolar com amplitude e primeiro momento apropriados é acionado antes dos gradientes de leitura de $k_{x}-k_{y}$, em cada aquisição. Posicionando os gradientes bipolares sobre o eixo $z$ irá codificar a velocidade perpendicular ao plano $x-y$. Posicionando os 
gradientes bipolares sobre $x$ ou $y$ irá codificar as velocidades no plano $x-y$. Escoamentos oblíquos podem ser codificados usando uma combinação de gradientes bipolares sobre as direções $x, y$ e $z$.

Cada aquisição sobre a direção $k_{w}$ é chamada de uma codificação de velocidade. O número necessário de codificações de velocidade depende da resolução de velocidade desejada e também do campo de visão de velocidade desejado (o alcance máximo de medidas de velocidade sem aliasing). Por exemplo, para obter $25 \mathrm{~cm} / \mathrm{s}$ de resolução com um campo de visão de 600 cm/s, 24 codificações de velocidade são necessários. A distribuição de velocidade $m(x, y, w)$ é obtida calculando a transformada de Fourier inversa dos dados adquiridos $M\left(k_{x}, k_{y}, k_{w}\right)$.

A principal desvantagem da CVF é o tempo de aquisição, pois $k_{x}-k_{y}$ precisa ser totalmente amostrado em cada valor de $k_{w}$. No entanto, já foram discutidos diferentes métodos para acelerar a CVF, como usar uma aquisição CVF com seleção de corte e codificação espacial em espiral [24].

\subsection{Modelo do Sinal de CVF}

Contraste de fase TF2D com codificação de velocidade somente ao longo do eixo $z$ fornece duas funções bidimensionais, $m(x, y)$ e $w_{\mathrm{cf}}(x, y)$, a magnitude e o mapa de velocidades, respectivamente. Se essas funções são adquiridas com uma resolução espacial suficientemente grande, e o escoamento é laminar, é possível assumir que cada voxel contém apenas uma velocidade e, portanto, a distribuição espacial de velocidade associado com o objeto é dada aproximadamente por:

$$
s(x, y, w)=m(x, y) \times \delta\left(w-w_{\mathrm{cf}}(x, y)\right),
$$

em que $\delta(z)$ é a função delta de Dirac.

Nas imagens CVF com aquisição cartesiana, ou TF2D, os dados no espaço $k$ são truncados num cubo retangular no espaço $k_{x}-k_{y}-k_{w}$. A distribuição espacial de velocidades associada pode ser modelada como sendo a convolução da distribuição real do objeto, $s(x, y, w), \operatorname{com} \operatorname{sinc}(x / \delta x), \operatorname{sinc}(y / \delta y)$ e $\operatorname{sinc}(w / \delta w)$, em que $\delta x$ e $\delta y$ são as resoluções espa- 
ciais sobre os eixos $x$ e $y$, respectivamente, e $\delta w$ é a resolução de velocidade. Ou seja:

$$
\hat{s}(x, y, w)=\left[m(x, y) \times \delta\left(w-w_{\mathrm{cf}}(x, y)\right)\right] * \operatorname{sinc}\left(\frac{x}{\delta x}\right) * \operatorname{sinc}\left(\frac{y}{\delta y}\right) * \operatorname{sinc}\left(\frac{w}{\delta w}\right)
$$

em que $\hat{s}(x, y, w)$ é a distribuição medida do objeto, e * denota a convolução. Isso é equivalente a:

$$
\hat{s}(x, y, w)=\left[m(x, y) \times \operatorname{sinc}\left(\frac{w-w_{\mathrm{cf}}(x, y)}{\delta w}\right)\right] *\left[\operatorname{sinc}\left(\frac{x}{\delta x}\right) \times \operatorname{sinc}\left(\frac{y}{\delta y}\right)\right] .
$$

\subsection{CVF com Aquisições em Espiral}

O imageamento de fluxo por contraste de fase é lento e possui limitações associadas aos efeitos de volume parcial, relação sinal-ruído e resolução temporal e espacial. Por outro lado, a CVF TF2D não possui essas limitações, no entanto necessita de tempos de leitura mais longos. Dessa forma, utilizamos o método de CVF com aquisições em espiral [23]. Esse método é capaz de adquirir dados totalmente localizados e distribuições de velocidade temporalmente resolvidas em um curto período de aquisição. Implementações para medir o fluxo sanguíneo em artérias carótidas e também comparações com a ultrasonografia e contraste de fase TF2D de alta resolução já foram apresentadas e discutidas por Carvalho et al. [23, 25].

\subsubsection{Sequências de Pulso}

A sequência de pulsos para o imageamento CVF em espiral (Fig. 2.6) consiste em uma seleção de corte, um gradiente bipolar de codificação de velocidade, uma codificação espacial em espiral e um gradiente de re-focalização. O conjunto de dados correspondente a cada quadro temporal é uma pilha de espirais no espaço $k_{x}-k_{y}-k_{w}$ (Fig. 2.7). O gradiente bipolar faz a codificação de fase efetivamente em $k_{w}$, enquanto cada espiral de leitura adquire um disco em $k_{x}-k_{y}$.

\subsubsection{Modelo de Sinal do CVF com Aquisições em Espiral}

Como as aquisições em espiral seguem um padrão de pilhas de espirais no espaço $k_{x}{ }^{-}$ $k_{y}-k_{w}$ (Fig. 2.7), o espaço $k$ é truncado num cilindro, i.e., um círculo ao longo do plano $k_{x}-k_{y}$ 


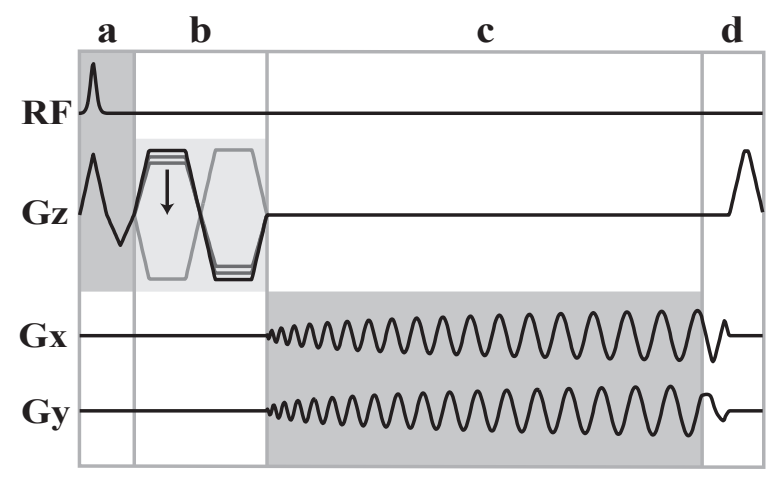

Figura 2.6: Sequência de pulsos da CVF em espiral. Essa consiste de (a) seleção de corte, (b) gradiente bipolar para codificação de velocidade, (c) codificação espacial em espiral, e (d) refocalização e anulação da magnetização.

(com diametro $1 / \delta r$ ), e uma função retangular (ou "porta") ao longo de $k_{w}$ (com espessura $1 / \delta w)$, em que $\delta r$ e $\delta w$ são as resoluções espaciais e de velocidade, respectivamente. Usando a mesma idéia da Eq. 2.18) (para CVF TF2D), a distribuição espacial de velocidades para a CVF em espiral pode ser modelada como a distribuição original, $s(x, y, w)$, convoluída com jinc $\left(\sqrt{x^{2}+y^{2}} / \delta r\right)$ e $\operatorname{sinc}(w / \delta w)$, resultando em:

$$
\begin{aligned}
\hat{s}(x, y, w) & =\left[m(x, y) \times \delta\left(w-w_{\mathrm{cf}}(x, y)\right)\right] * \operatorname{jinc}\left(\frac{\sqrt{x^{2}+y^{2}}}{\delta r}\right) * \operatorname{sinc}\left(\frac{w}{\delta w}\right) \\
& =\left[m(x, y) \times \operatorname{sinc}\left(\frac{w-w_{\mathrm{cf}}(x, y)}{\delta w}\right)\right] * \operatorname{jinc}\left(\frac{\sqrt{x^{2}+y^{2}}}{\delta r}\right) .
\end{aligned}
$$

\subsection{Conclusão}

Foram introduzidos os princípios básicos do imageamento por ressonância magnética e também apresentada uma breve revisão do imageamento do escoamento sanguíneo utilizando a ressonância magnética. Os métodos de imageamento de fluxo, contraste de fase e codificação de velocidade por Fourier são parte fundamental deste trabalho. Mapas de velocidade obtido com contraste de fase serão utilizados para guiar soluções computacionais da dinâmica dos fluidos e a modelagem do sinal da distribuição de velocidades adquirida com CVF será a base para os resultados apresentados no Capítulo 5, em que será discutida a possibilidade de obtenção de um mapa de velocidades a partir das distribuições de 


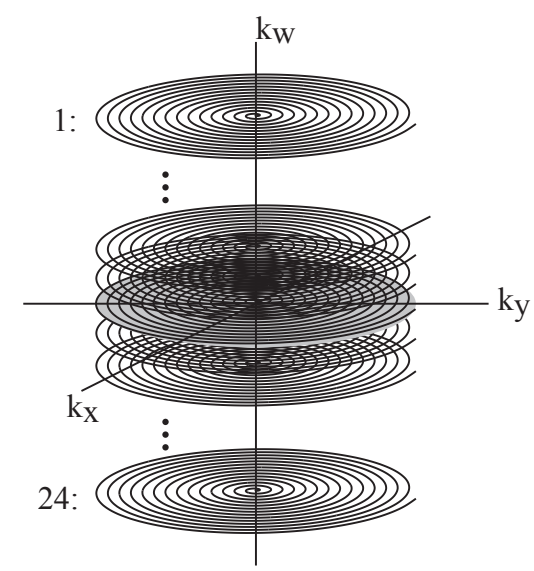

Figura 2.7: Esquema de amostragem do espaço $k$ para o CVF em espiral. O conjunto de dados correspondente a cada quadro temporal é uma pilha de espirais no espaço $k_{x}-k_{y}-k_{w}$. Cada aquisição em espiral corresponde a um nível diferente de codificação em $k_{w}$.

velocidades CVF de baixa resolução espacial e também simulações computacionais usando dados adquiridos com CVF. 


\section{Capítulo 3}

\section{Dinâmica dos Fluidos Computacional}

Neste capítulo faremos a descrição do algoritmo SIMPLER (Semi-Implicit Method for Pressure Linked Equations Revised), desenvolvido por Patankar \& Spalding [55, utilizando o método dos volumes finitos. Este algoritmo é amplamente utilizado tanto academicamente quanto comercialmente para determinar soluções numéricas das equações de NavierStokes, que modelam o escoamento de um fluido. A descrição do algoritmo SIMPLER se faz importante, pois o mesmo será utilizado como base para a construção das simulações computacionais guiadas por ressonância magnética. Neste trabalho, o algoritmo implementado é destinado a simulação de escoamentos tridimensionais entretanto, por uma questão didática, será apresentada neste capítulo a dedução da versão bidimensional do algoritmo SIMPLER. A dedução do algoritmo na versão tridimensional é análoga a versão bidimensional e segue de forma direta. Além disso, com o intuito de efetuar a validação do algoritmo implementado, foi utilizado o problema do escoamento bidimensional em uma cavidade, que é conhecido na literatura por ser um teste padrão para algoritmos de dinâmica dos fluidos computacional.

Neste trabalho, o sangue será modelado como sendo um fluido Newtoniano incompressível isotérmico, com densidade constante $\rho$ e viscosidade constante $\mu$. Apesar de estarmos supondo a viscosidade do sangue como uma constante, é fato bem conhecido que o sangue é um fluido não-Newtoniano, logo, a sua viscosidade não é uniforme. Na literatura existem diversos modelos constitutivos e estudos relacionados às propriedades reológicas não-Newtonianos do sangue [64, 65, 66]. No entanto, a falta de um modelo constitutivo estabelecido, a facilidade de implementação do modelo Newtoniano e o uso corriqueiro da 
viscosidade constante do sangue na literatura [36, 25, 34, 43, 46, 32] nos fizeram optar pelo uso do modelo de escoamento sanguíneo Newtoniano.

Faz-se importante salientar que os efeitos elásticos das paredes dos vasos também não estão sendo considerados neste trabalho. Aqui estamos analisando dados de CF e CVF de um phantom de escoamento pulsátil, cuja parede do tubo é rígida, e dados de CF in vivo, cujas paredes dos vasos são elásticas. A hipótese de se considerar vasos com paredes rígidas em simulações da DFC também é comumente utilizada na literatura [54, 34, 43, 46, 32], apesar de já existirem simulações da DFC do escoamento sanguíneo considerando os efeitos elásticos de parede [67, 68]. No entanto, o algoritmo SIMPLER, com malha uniforme Cartesiana, implementado nesse estudo não permite a utilização do modelo de paredes elásticas. Para utilizarmos essa hipótese, seria necessário uma implementação através do método dos elementos finitos, que nos permitiria os efeitos de interação fluido-estrutura. Porém, tal implementação é substancialmente mais complexa do que o algoritmo que foi implementado neste trabalho.

\subsection{Equações do Movimento}

O modelo geral do movimento de um fluido no espaço tridimensional é dado pela equação do momentum de Navier-Stokes [64]:

$$
\rho\left(\frac{\partial \vec{\nu}}{\partial t}+\vec{\nu} \cdot \nabla \vec{\nu}\right)=-\nabla p-\nabla \cdot \hat{\tau}+\vec{f}
$$

em que $\rho$ é a densidade do fluido, $\vec{\nu}=(u, v, w)$ é o vetor velocidade do escoamento $(u$, $v$ e $w$ são as componentes de velocidade escalares associadas aos eixos espaciais $x, y$ e $z$, respectivamente), $t$ é o tempo, $p$ é a pressão hidrodinâmica, $\vec{f}$ representa as forças de campo agindo sobre o fluido durante o escoamento, $\hat{\tau}$ é o tensor de tensões, e $\nabla$ é o operador gradiente. O tensor de tensões representa o momentum transferido em virtude dos movimentos moleculares e interações dentro do fluido. Ele é uma função das invariantes escalares do tensor taxa de deformação $\hat{e}=(1 / 2)\left[\nabla \vec{\nu}+(\nabla \vec{\nu})^{T}\right]$, em que ${ }^{T}$ é a operação de transposição. Para um fluido incompressível, o tensor de tensões pode ser escrito como $\hat{\tau}=-\mu(\hat{e}) \hat{e}$, em que o escalar $\mu(\hat{e})$ é a viscosidade Newtoniana generalizada para um dado $\hat{e}$. 
Neste trabalho, o sangue é modelado com um fluido Newtoniano, incompressível, isotérmico, com viscosidade constante $\mu$ e densidade constante $\rho$. Estamos supondo também que não há nenhuma força de campo agindo sobre o escoamento do sangue. Desta forma, a simplificação da equação do momentum geral, Eq. (3.1), leva a equação para o movimento do sangue [69]:

$$
\rho\left(\frac{\partial \vec{\nu}}{\partial t}+\vec{\nu} \cdot \nabla \vec{\nu}\right)=-\nabla p+\mu \Delta \vec{\nu}
$$

em que $\Delta$ é o operador Laplaciano.

Além disso, como não há fontes de sangue no interior das artérias, o campo de velocidades também deve satisfazer a conservação da massa (ou continuidade), que pode ser expressa por:

$$
\nabla \cdot \vec{\nu}=0
$$

As Eq. (3.2) e Eq. (3.3) devem ser resolvidas para as variáveis escalares $u, v, w$ e $p$. Essas equações formam um sistema acoplado não linear de equações diferenciais parciais. A tentativa de determinar sua solução geral diretamente é um problema em aberto e, certamente, uma tarefa formidável. Desta forma, é necessário um método para encontrar soluções aproximadas desse sistema. Os métodos que serão descritos neste capítulo serão baseados, basicamente, nas discretizações por diferenças finitas em volumes de controle definidos na malha computacional. Além disso, será deduzida uma equação de Poisson, baseada na equação da continuidade (Eq. (3.3)), para determinar a pressão. Nas seções seguintes serão apresentados os detalhes da discretização e o algoritmo de aproximação da equação de Navier-Stokes.

\subsection{Malha Deslocada (Staggered Grid)}

A maior dificuldade encontrada na determinação da solução numérica de um escoamento incompressível é a falta de uma equação direta para a pressão. Essa dificuldade pode ser solucionada facilmente para escoamentos bidimensionais utilizando uma abordagem em funções de corrente na qual a pressão é eliminada das equações do momentum (Eq. (3.2)). No entanto, essa abordagem não é possível em um escoamento tridimensional [70]. Desta forma, problemas tridimensionais demandam uma abordagem utilizando as variáveis primitivas $u, v, w$ e $p$. No passado, muitos pesquisadores fizeram esforços para que os problemas 


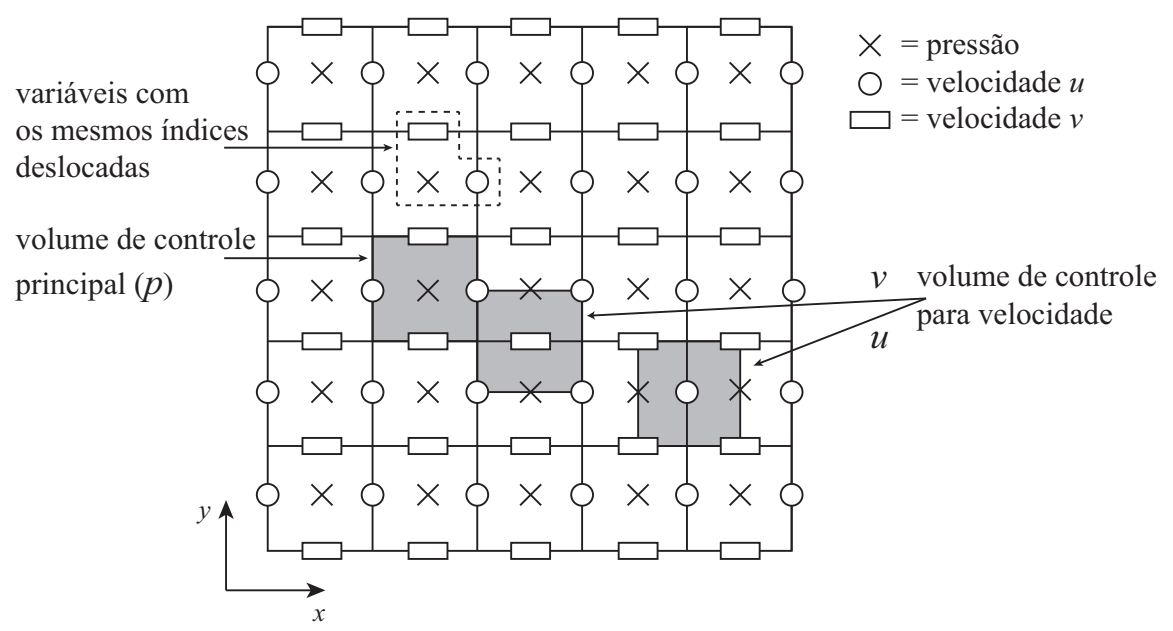

Figura 3.1: Malha deslocada.

bidimensionais e tridimensionais pudessem ser solucionados utilizando apenas as variáveis primitivas, sem que o campo de pressões apresentasse oscilações não físicas. Como solução, foi sugerido utilizar uma malha de cálculo diferente para cada uma das variáveis dependentes. Uma malha deslocada para as variáveis dependentes foi primeiramente utilizada por Harlow \& Welch [71], no seu bem conhecido método MAC (Marker and Cell). Desde então, esse método vem sendo utilizado por diversos pesquisadores como um padrão, especificamente, no algoritmo SIMPLER, procedimento criado e popularizado por Patankar \& Spalding [47].

A Fig. 3.1 mostra uma malha bidimensional deslocada em que as variáveis dependentes $\left(u_{i, j}, v_{i, j}\right.$ e $\left.p_{i, j}\right)$ com os mesmos índices são deslocadas umas das outras (uma extensão para três dimensões é simples e direta). O domínio computacional é dividido em um número de células (mostradas na Fig. 3.1 como volume de controle principal) cujos valores da pressão estão no centro. A localização das componentes de velocidade fica no centro das faces das células nas quais elas são normais, i.e., a velocidade $u$ fica no ponto médio das faces verticais e a velocidade $v$ no ponto médio da face horizontal. Se uma malha uniforme é usada, as localizações são exatamente os ponto médios desses pontos de malha. Em tais casos, a diferença de pressão entre duas células adjacentes é a força motriz para a componente de velocidade localizada na interface dessas células. Dessa forma, a aproximação por diferenças finitas é agora fisicamente significativa e o campo de pressão irá produzir uma distribuição de pressão razoável para o campo de velocidade correto.

Outra importante vantagem é que as taxas de transporte transversalmente às faces do 
volume de controle podem ser calculadas sem a necessidade de interpolação das componentes de velocidade. Uma descrição detalhada de como determinar as equações discretizadas de Navier-Stokes usando uma malha deslocada será feita nas seções subsequentes.

\subsection{Discretização das Equações do Movimento 2D}

O algoritmo SIMPLER é baseado na discretização das equações de Navier-Stokes. Por motivos didáticos, a discretização será apresentada para as equações bidimensionais do movimento em uma malha computacional uniforme e Cartesiana. A discretização para o caso tridimensional pode ser feita trivialmente de forma análoga e será apresentada ao final da dedução da discretização.

Discretizamos o espaço $x-y-t$ em uma malha com espaçamento espacial $\delta x, \delta y$ e resolução temporal $\delta t^{1}$. Além disso, definimos os pontos discretos da malha $\left(x_{i}, y_{j}, t_{n}\right)$ por:

$$
\begin{aligned}
& x_{i}=i \delta x, i \in \mathbb{N} \\
& y_{j}=j \delta y, \quad j \in \mathbb{N} \\
& t_{n}=n \delta t, \quad n \in \mathbb{N} .
\end{aligned}
$$

O nosso interesse é determinar as funções escalares de velocidade $u(x, y, t)$ e $v(x, y, t)$ e a pressão $p(x, y, t)$ nos pontos da malha discreta $\left(x_{i}, y_{j}, t_{n}\right)$ e, para tais, escreveremos:

$$
\begin{aligned}
u\left(x_{i}, y_{j}, t_{n}\right) & =u_{i, j}^{n} \\
v\left(x_{i}, y_{j}, t_{n}\right) & =v_{i, j}^{n} \\
p(x, y, t) & =p_{i, j}^{n} .
\end{aligned}
$$

Para um escoamento bidimensional a componente $w$ da velocidade é nula e ,neste caso, a equação da continuidade pode ser escrita como:

$$
\frac{\partial u}{\partial x}+\frac{\partial v}{\partial y}=0
$$

\footnotetext{
${ }^{1}$ É importante observar que a resolução temporal $\delta t$ é diferente da resolução temporal da imagem que será adquirida por ressonância magnética. Neste caso, a resolução temporal está relacionada a solução numérica que irá convergir para o estado estacionário, i.e., $\vec{\nu}_{\infty}(\vec{r})=\lim _{t \rightarrow \infty} \vec{\nu}(\vec{r}, t)$, levando em consideração as velocidades dadas como condições de entrada e saída.
} 
Para o volume de controle mostrado na Fig. 3.2, a aplicação do método dos volumes finitos na equação da continuidade, i.e.,

$$
\iint\left(\frac{\partial u}{\partial x}+\frac{\partial v}{\partial y}\right) d x d y
$$

produzirá a seguinte forma discretizada da equação da continuidade:

$$
\left(u_{i, j}^{n+1}-u_{i-1, j}^{n+1}\right) \delta y+\left(v_{i, j}^{n+1}-v_{i, j-1}^{n+1}\right) \delta x=0 .
$$

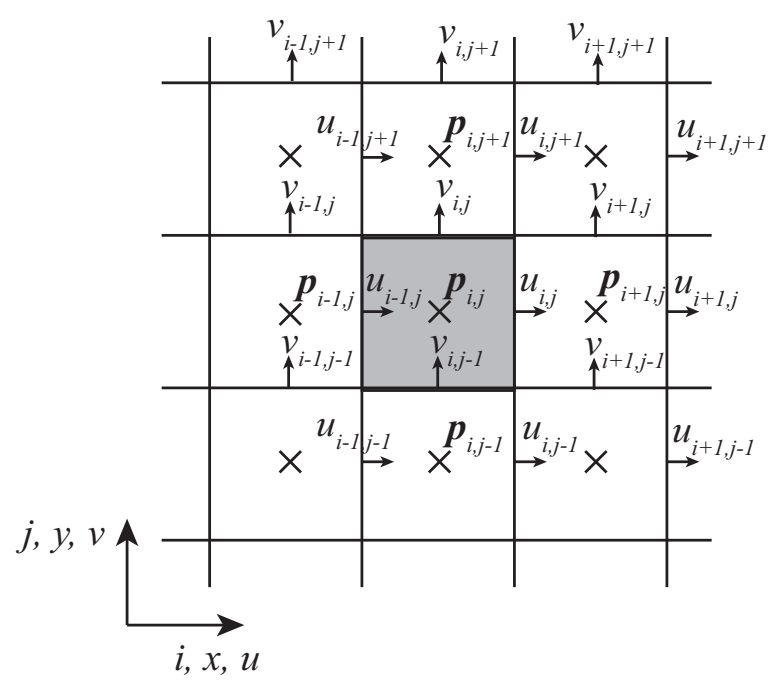

Figura 3.2: Volume de controle para a equação da continuidade.

\subsubsection{Equação do Momentum na Direção $x$}

A equação de Navier-Stokes bidimensional na direção $x$, na forma conservativa (com as devidas simplificações baseadas na equação da continuidade), é dada por:

$$
\rho\left[\frac{\partial u}{\partial t}+\frac{\partial(u u)}{\partial x}+\frac{\partial(u v)}{\partial y}\right]=-\frac{\partial p}{\partial x}+\mu\left[\frac{\partial^{2} u}{\partial x^{2}}+\frac{\partial^{2} u}{\partial y^{2}}\right] .
$$

Integrando a Eq. (3.9) sobre o volume de controle para a variável dependente $u$ (Fig. 3.3), temos:

$$
\begin{array}{r}
\rho\left(u_{i, j}^{n+1}-u_{i, j}^{n}\right) \frac{\delta x \delta y}{\delta t}+\iint\left(\frac{\partial p}{\partial x}\right) d x d y \\
\iint\left[\frac{\partial}{\partial x}\left(\rho u u-\mu \frac{\partial u}{\partial x}\right)+\frac{\partial}{\partial y}\left(\rho u v-\mu \frac{\partial u}{\partial y}\right)\right] d x d y=0
\end{array}
$$


em que $\delta x$ e $\delta y$ representam as dimensões do volume de controle, $\delta t$ a resolução temporal e $u^{n}$ a componente da velocidade no tempo $t=n \delta t$. A aplicação do teorema de Green na Eq. (3.10) leva a seguinte equação discretizada:

$$
\begin{aligned}
\rho\left(u_{i, j}^{n+1}-u_{i, j}^{n}\right) \frac{\delta x \delta y}{\delta t}+\left(G_{i+\frac{1}{2}, j}^{x}-G_{i-\frac{1}{2}, j}^{x}\right) \delta y & + \\
\left(H_{i, j+\frac{1}{2}}^{x}-H_{i, j-\frac{1}{2}}^{x}\right) \delta x+\left(p_{i+1, j}^{n+1}-p_{i, j}^{n+1}\right) \delta y & =0
\end{aligned}
$$

em que $G^{x}$ e $H^{x}$ são definidos como:

$$
G^{x}=\rho u u-\mu \frac{\partial u}{\partial x} \text { e } H^{x}=\rho u v-\mu \frac{\partial u}{\partial y}
$$

As variáveis $G^{x}$ e $H^{x}$ representam os fluxos axial e transversal para a equação do momentum na direção $x$, respectivamente. As discretizações dos fluxos são diretas e dadas por:

$$
\begin{aligned}
G_{i+\frac{1}{2}, j}^{x} & =\frac{\rho}{4}\left(u_{i, j}^{n}+u_{i+1, j}^{n}\right)\left(u_{i, j}^{n+1}+u_{i+1, j}^{n+1}\right)-\mu \frac{\left(u_{i+1, j}^{n+1}-u_{i, j}^{n+1}\right)}{\delta x} \\
G_{i-\frac{1}{2}, j}^{x} & =\frac{\rho}{4}\left(u_{i-1, j}^{n}+u_{i, j}^{n}\right)\left(u_{i-1, j}^{n+1}+u_{i, j}^{n+1}\right)-\mu \frac{\left(u_{i, j}^{n+1}-u_{i-1, j}^{n+1}\right)}{\delta x} \\
H_{i, j+\frac{1}{2}}^{x} & =\frac{\rho}{4}\left(v_{i, j}^{n}+v_{i+1, j}^{n}\right)\left(u_{i, j}^{n+1}+u_{i, j+1}^{n+1}\right)-\mu \frac{\left(u_{i, j+1}^{n+1}-u_{i, j}^{n+1}\right)}{\delta y} \\
H_{i, j-\frac{1}{2}}^{x} & =\frac{\rho}{4}\left(v_{i, j-1}^{n}+v_{i+1, j-1}^{n}\right)\left(u_{i, j-1}^{n+1}+u_{i, j}^{n+1}\right)-\mu \frac{\left(u_{i, j}^{n+1}-u_{i, j-1}^{n+1}\right)}{\delta y}
\end{aligned}
$$

Consequentemente, substituindo os fluxos discretizados na Eq. (3.11), verifica-se que a mesma pode ser reescrita na forma:

$$
a_{i, j}^{u} u_{i, j}^{n+1}=\sum a_{n b}^{u} u_{n b}^{n+1}+b^{u}+\left(p_{i, j}^{n+1}-p_{i+1, j}^{n+1}\right) \delta y
$$

em que o termo $\sum a_{n b}^{u} u_{n b}^{n+1}$ representa todas as contribuições convectivas e difusivas nos nós vizinhos $\left(u_{i+1, j}, u_{i-1, j}, u_{i, j+1}, u_{i, j-1}\right.$ e seus coeficientes), e os coeficientes $a_{i, j}^{u}$ e $a_{n b}^{u}$ contêm todas as informações do tamanho da malha, densidade, viscosidade e também a solução de $u$ e $v$ no $n$-ésimo tempo. Desta forma, reescrevendo a Eq. 3.14, temos:

$$
a_{i, j}^{u} u_{i, j}^{n+1}=a_{i-1, j}^{u} u_{i-1, j}^{n+1}+a_{i+1, j}^{u} u_{i+1, j}^{n+1}+a_{i, j-1}^{u} u_{i, j-1}^{n+1}+a_{i, j+1}^{u} u_{i, j+1}^{n+1}+b^{u}+\left(p_{i, j}^{n+1}-p_{i+1, j}^{n+1}\right) \delta y,
$$


em que os coeficientes são dados por:

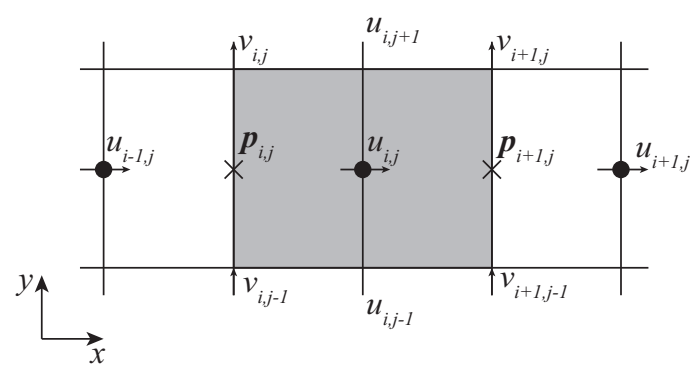

Figura 3.3: Volume de controle para a equação do momentum na direção $x$.

$$
\begin{aligned}
a_{i-1, j}^{u} & =D_{i-1, j}^{u}+\frac{F_{i-1, j}^{u}}{2} \\
a_{i+1, j}^{u} & =D_{i+1, j}^{u}-\frac{F_{i+1, j}^{u}}{2} \\
a_{i, j-1}^{u} & =D_{i, j-1}^{u}+\frac{F_{i, j-1}^{u}}{2} \\
a_{i, j+1}^{u} & =D_{i, j+1}^{u}-\frac{F_{i, j+1}^{u}}{2} \\
b^{u} & =\rho \frac{\delta x \delta y}{\delta t} u_{i, j}^{n} \\
a_{i, j}^{u} & =a_{i-1, j}^{u}+a_{i+1, j}^{u}+a_{i, j-1}^{u}+a_{i, j+1}^{u} \\
& +\left(F_{i+1, j}^{u}-F_{i-1, j}^{u}\right)+\left(F_{i, j+1}^{u}-F_{i, j+1}^{u}\right)+\rho \frac{\delta x \delta y}{\delta t} .
\end{aligned}
$$

Neste caso, os coeficientes $D$ representam as contribuições difusivas e os coeficientes $F$ as contribuições convectivas, com:

$$
\begin{aligned}
D_{i-1, j}^{u} & =D_{i+1, j}^{u}=\mu \frac{\delta y}{\delta x}, \\
D_{i, j-1}^{u} & =D_{i, j+1}^{u}=\mu \frac{\delta x}{\delta y}, \\
F_{i-1, j}^{u} & =\frac{1}{2}\left(u_{i-1, j}^{n}+u_{i, j}^{n}\right) \delta y, \\
F_{i+1, j}^{u} & =-\frac{1}{2}\left(u_{i+1, j}^{n}+u_{i, j}^{n}\right) \delta y, \\
F_{i, j-1}^{u} & =\frac{1}{2}\left(v_{i, j-1}^{n}+v_{i+1, j-1}^{n}\right) \delta x, \\
F_{i+1, j}^{u} & =-\frac{1}{2}\left(v_{i+1, j}^{n}+v_{i, j}^{n}\right) \delta x .
\end{aligned}
$$




\subsubsection{Equação do Momentum na Direção $y$}

A equação do momentum na direção $y$ é dada por:

$$
\rho\left[\frac{\partial v}{\partial t}+\frac{\partial(u v)}{\partial x}+\frac{\partial(v v)}{\partial y}\right]=-\frac{\partial p}{\partial y}+\mu\left[\frac{\partial^{2} v}{\partial x^{2}}+\frac{\partial^{2} v}{\partial y^{2}}\right]
$$

E de forma análoga ao procedimento da seção anterior, podemos integrar sobre o volume de controle para a velocidade $v$ (Fig. 3.4), obtendo que:

$$
\begin{array}{r}
\rho\left(v_{i, j}^{n+1}-v_{i, j}^{n}\right) \frac{\delta x \delta y}{\delta t}+\iint\left(\frac{\partial p}{\partial y}\right) d x d y \\
\iint\left[\frac{\partial}{\partial x}\left(\rho u v-\mu \frac{\partial v}{\partial x}\right)+\frac{\partial}{\partial y}\left(\rho v v-\mu \frac{\partial v}{\partial y}\right)\right] d x d y=0 .
\end{array}
$$

Novamente, aplicando o teorema de Green na equação, obtemos:

$$
\begin{aligned}
& \rho\left(v_{i, j}^{n+1}-v_{i, j}^{n}\right) \frac{\delta x \delta y}{\delta t}+\left(G_{i+\frac{1}{2}, j}^{y}-G_{i-\frac{1}{2}, j}^{y}\right) \delta y \\
& \quad\left(H_{i, j+\frac{1}{2}}^{y}-H_{i, j-\frac{1}{2}}^{y}\right) \delta x+\left(p_{i, j+1}^{n+1}-p_{i, j}^{n+1}\right) \delta x=0
\end{aligned}
$$

em que os fluxos axial e transversal na direção $y, G^{y}$ e $H^{y}$, são definidos como:

$$
G^{y}=\rho u v-\mu \frac{\partial v}{\partial x} \text { e } H^{y}=\rho v v-\mu \frac{\partial v}{\partial y} .
$$

E, repetindo o procedimento da seção anterior, a discretização dos fluxos pode ser determinada com facilidade:

$$
\begin{aligned}
G_{i+\frac{1}{2}, j}^{y} & =\frac{\rho}{4}\left(u_{i, j}^{n}+u_{i, j+1}^{n}\right)\left(v_{i, j}^{n+1}+v_{i+1, j}^{n+1}\right)-\mu \frac{\left(v_{i+1, j}^{n+1}-v_{i, j}^{n+1}\right)}{\delta x} \\
G_{i-\frac{1}{2}, j}^{y} & =\frac{\rho}{4}\left(u_{i-1, j}^{n}+u_{i-1, j+1}^{n}\right)\left(v_{i-1, j}^{n+1}+v_{i, j}^{n+1}\right)-\mu \frac{\left(v_{i, j}^{n+1}-v_{i-1, j}^{n+1}\right)}{\delta x} \\
H_{i, j+\frac{1}{2}}^{y+1} & =\frac{\rho}{4}\left(v_{i, j}^{n}+v_{i, j+1}^{n}\right)\left(v_{i, j}^{n+1}+v_{i, j+1}^{n+1}\right)-\mu \frac{\left(v_{i, j+1}^{n+1}-v_{i, j}^{n+1}\right)}{\delta y} \\
H_{i, j-\frac{1}{2}}^{y} & =\frac{\rho}{4}\left(v_{i, j-1}^{n}+v_{i, j}^{n}\right)\left(v_{i, j-1}^{n+1}+v_{i, j}^{n+1}\right)-\mu \frac{\left(v_{i, j}^{n+1}-v_{i, j-1}^{n+1}\right)}{\delta y} .
\end{aligned}
$$




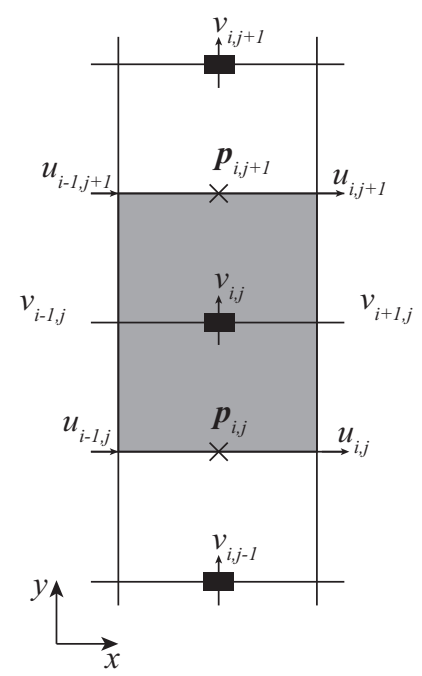

Figura 3.4: Volume de controle para a equação do momentum na direção $y$.

Finalmente, a Eq. 3.20 pode ser escrita como:

$a_{i, j}^{v} u_{i, j}^{n+1}=a_{i-1, j}^{v} v_{i-1, j}^{n+1}+a_{i+1, j}^{v} v_{i+1, j}^{n+1}+a_{i, j-1}^{v} v_{i, j-1}^{n+1}+a_{i, j+1}^{v} v_{i, j+1}^{n+1}+b^{v}+\left(p_{i, j}^{n+1}-p_{i, j+1}^{n+1}\right) \delta x$,

em que os coeficientes são dados por:

$$
\begin{aligned}
a_{i-1, j}^{v}=D_{i-1, j}^{v}+\frac{F_{i-1, j}^{v}}{2} & a_{i+1, j}^{v}=D_{i+1, j}^{v}-\frac{F_{i+1, j}^{v}}{2} \\
a_{i, j-1}^{v}= & D_{i, j-1}^{v}+\frac{F_{i, j-1}^{v}}{2} \\
a_{i, j+1}^{v}= & D_{i, j+1}^{v}-\frac{F_{i, j+1}^{v}}{2} \\
b^{v}= & \rho \frac{\delta x \delta y}{\delta t} v_{i, j}^{n} \\
a_{i, j}^{v}= & a_{i-1, j}^{v}+a_{i+1, j}^{v}+a_{i, j-1}^{v}+a_{i, j+1}^{v} \\
+ & \left(F_{i+1, j}^{v}-F_{i-1, j}^{v}\right)+\left(F_{i, j+1}^{v}-F_{i, j+1}^{v}\right)+\rho \frac{\delta x \delta y}{\delta t},
\end{aligned}
$$


com:

$$
\begin{aligned}
D_{i-1, j}^{v} & =D_{i+1, j}^{v}=\mu \frac{\delta y}{\delta x}, \\
D_{i, j-1}^{v} & =D_{i, j+1}^{v}=\mu \frac{\delta x}{\delta y}, \\
F_{i-1, j}^{v} & =\frac{1}{2}\left(u_{i-1, j}^{n}+u_{i-1, j+1}^{n}\right) \delta y \\
F_{i+1, j}^{v} & =-\frac{1}{2}\left(u_{i, j+1}^{n}+u_{i, j}^{n}\right) \delta y, \\
F_{i, j-1}^{v} & =\frac{1}{2}\left(v_{i, j-1}^{n}+v_{i, j}^{n}\right) \delta x, \\
F_{i+1, j}^{v} & =-\frac{1}{2}\left(v_{i, j+1}^{n}+v_{i, j}^{n}\right) \delta x .
\end{aligned}
$$

\subsubsection{Procedimento de Solução}

Em um estágio intermediário do funcionamento do algoritmo que calcula a solução computacional, as variáveis dependentes deverão evoluir no tempo computacional passando do instante de tempo $n \delta t$ para o instante $(n+1) \delta t$. No SIMPLER, a velocidade é determinada em duas etapas - predição e correção - para prosseguir do n-ésimo intervalo de tempo computacional para o seguinte. No primeiro momento, as equações do momentum discretizadas Eq. (3.15) e Eq. 3.23) são resolvidas para obter valores provisórios para as velocidades que serão chamadas de $u^{*}$ e $v^{*}$. Não será possível obter as velocidades $u^{n+1} \mathrm{e}$ $v^{n+1}$ diretamente, pois os valores provisórios, no primeiro momento, não irão satisfazer a equação da continuidade.

Fazendo uso das soluções aproximadas das velocidades $u^{*}$ e $v^{*}$, uma correção para a pressão $\delta p$ poderá ser calculada, o que nos dará uma equação para a correção da pressão $p^{n+1}=p^{n}+\delta p$ e, com essa nova pressão, correções para as velocidade $u^{c}$ e $v^{c}$ serão obtidas. Como no caso da pressão, $u^{c}$ irá corrigir $u^{*}$ de tal forma que $u^{n+1}=u^{*}+u^{c}$, e equivalentemente para $v^{c}$, sendo que os novos valores $u^{n+1}$ e $v^{n+1}$ satisfazerão a equação da continuidade.

Assim, com o intuito de obter os valores provisórios $u^{*}$ e $v^{*}$, as equações Eq. (3.11) e Eq. (3.20) são aproximadas por: 


$$
\begin{aligned}
& a_{i, j}^{u} u_{i, j}^{*}=\sum a_{n b}^{u} u_{n b}^{*}+b^{u}+\left(p_{i, j}^{n}-p_{i+1, j}^{n}\right) \delta y \\
& a_{i, j}^{v} v_{i, j}^{*}=\sum a_{n b}^{v} v_{n b}^{*}+b^{v}+\left(p_{i, j}^{n}-p_{i, j+1}^{n}\right) \delta x .
\end{aligned}
$$

Observe que o campo de pressão utilizado é o obtido na etapa de tempo anterior. Sendo $p^{n}$ conhecido, as equações Eq. 3.26 e Eq. 3.27 podem ser resolvidas. Por exemplo, na equação, a velocidade $u_{i, j}^{*}$ e todos os vizinhos $u_{i+1, j}^{*}, u_{i-1, j}^{*}, u_{i, j+1}^{*}, u_{i, j-1}^{*}$ são desconhecidos e podem ser expressos como um sistema de equações lineares. Isto envolve a inversão de uma matriz banda com cinco diagonais.

Agora, considerando que $u^{n+1}=u^{*}+u^{c}$, se subtrairmos a Eq. 3.15 da Eq. 3.26, temos:

$$
a_{i, j}^{u} u_{i, j}^{c}=\sum a_{n b}^{u} u_{n b}^{c}+\left(\delta p_{i, j}-\delta p_{i+1, j}\right) \delta y
$$

$\operatorname{com} \delta p_{i, j}=p_{i, j}^{n+1}-p_{i, j}^{n}$. De uma forma similar, subtraindo a Eq. 3.23 da Eq. 3.27 produzimos a equação de correção $v^{c}$ :

$$
a_{i, j}^{v} v_{i, j}^{c}=\sum a_{n b}^{v} v_{n b}^{c}+\left(\delta p_{i, j}-\delta p_{i, j+1}\right) \delta x
$$

Com o objetivo de se fazer a ligação entre as correções $u^{c}$ e $v^{c}$ e a correção da pressão $\delta p$ de forma explícita, os termos que contêm todas as vizinhanças do ponto central $(i, j)$ serão ignorados nas Eqs. 3.28) e 3.29) (na Ref. [47] é possível encontrar a justificativa completa para esta operação). Desta forma, as mesmas serão reduzidas a:

$$
u_{i, j}^{c}=d_{i, j}^{u}\left(\delta p_{i, j}-\delta p_{i+1, j}\right)
$$

em que $d_{i, j}^{u}=\frac{\delta y}{a_{i, j}^{u}}, \mathrm{e}$

$$
v_{i, j}^{c}=d_{i, j}^{v}\left(\delta p_{i, j}-\delta p_{i, j+1}\right)
$$

$\operatorname{com} d_{i, j}^{v}=\frac{\delta x}{a_{i, j}^{v}}$

Agora, substituindo $u_{i, j}^{n+1}=u_{i, j}^{*}+u_{i, j}^{c}$ e $v_{i, j}^{n+1}=v_{i, j}^{*}+v_{i, j}^{c}$ na equação da continuidade discretizada (Eq. (3.8) ) e usando as Eqa. (3.28) e (3.29), temos a equação da correção da pressão:

$$
a_{i, j}^{p} \delta p_{i, j}=\sum a_{n p}^{p} \delta p_{n b}+b^{p}
$$


em que $b^{p}=\left(u_{i, j}^{*}-u_{i-1, j}^{*}\right) \delta y+\left(v_{i, j}^{*}-v_{i, j-1}^{*}\right) \delta x$. Considerando $\vec{\nu}^{*}=\left(u^{*}, v^{*}\right)$, então a Eq. (3.32) é, basicamente, uma versão discreta da seguinte equação de Poisson:

$$
\nabla(\delta p)=\frac{1}{\delta t} \nabla \cdot \vec{\nu}^{*}
$$

É importante notar que a solução numérica converge quando o termo $b^{p}=0$. Vale observar também que as Eqs. 3.30 e 3.31 podem ser representadas como:

$$
\vec{\nu}^{c}=-\frac{1}{\delta t} \nabla(\delta p)
$$

Finalmente, os coeficientes da equação da pressão (Eq. (3.32) são dados por:

$$
\begin{aligned}
a_{i+1, j}^{p} & =d_{i+1, j}^{u} \delta y \\
a_{i-1, j}^{p} & =d_{i-1, j}^{u} \delta y \\
a_{i, j+1}^{p} & =d_{i, j+1}^{v} \delta x \\
a_{i, j-1}^{p} & =d_{i, j-1}^{v} \delta x \\
a_{i, j}^{p} & =a_{i-1, j}^{P}+a_{i+1, j}^{P}+a_{i, j-1}^{P}+a_{i, j+1}^{P} \\
b^{p} & =\left(u_{i, j}^{*}-u_{i-1, j}^{*}\right) \delta y+\left(v_{i, j}^{*}-v_{i, j-1}^{*}\right) \delta x .
\end{aligned}
$$

\subsubsection{Algoritmo}

Com o objetivo de sistematizar o procedimento discutido nas seções anteriores apresentaremos nesta seção os algoritmos para determinar a solução numérica das equações de Navier-Stokes. Começaremos apresentando o método SIMPLE (Alg. 1)

Com o objetivo de melhorar a taxa de convergência do algoritmo SIMPLE, uma versão revista, chamada de SIMPLER, ou SIMPLE Revised, foi sugerida por Patankar [47]. Para o nosso caso, esta versão é de maior interesse, pois ela não necessita de uma estimativa inicial para a pressão, mas apenas de uma estimativa inicial para a velocidade. Levando em consideração que possuímos, em um determinado vaso de interesse, um campo de velocidades medido por contraste de fase (mesmo que de baixa resolução espacial e ruidoso), podemos utilizá-lo como estimativa inicial. Além disso, se o campo de velocidades correto é dado, a versão da equação da pressão para o algoritmo SIMPLER irá produzir imediatamente o campo de pressão correto e não serão necessárias novas iterações. Apesar do algoritmo 
1. Comece com uma estimativa inicial para o campo de pressão, $p^{*}$.

2. Determine a solução das equações discretizadas do movimento, Eq. (3.15) e Eq. (3.23), para obter $u^{*}$ e $v^{*}$.

3. Determine a solução para a equação da correção da pressão, $\delta p$.

4. Calcule a correção da pressão: $p^{n+1}=p^{*}+\delta p$.

5. Determine $u^{n+1}$ e $v^{n+1}$ usando as fórmulas de correção da velocidade

$$
\begin{aligned}
u^{n+1} & =u^{*}+d^{u}\left(\delta p_{i}-\delta p_{i+1}\right) \\
v^{n+1} & =v^{*}+d^{v}\left(\delta p_{j}-\delta p_{j+1}\right) .
\end{aligned}
$$

6. Trate a pressão corrigida, $p^{n+1}$, como a nova estimativa inicial, i.e., $p^{*}=p^{n+1}$. Retorne ao passo 2 e repita todo o procedimento até a equação discreta da continuidade ser satisfeita.

SIMPLER possuir uma taxa de convergência mais rápida, é importante ressaltar que o mesmo tem um esforço computacional maior que o SIMPLE [47].

Com o objetivo de determinar a versão revisada do algoritmo SIMPLE, considere que seja conhecido um campo de velocidades inicial $u$ e $v$. Então, a equação do movimento (Eq. (3.15)) pode ser escrita como:

$$
u_{i, j}=\frac{\sum a_{n b}^{u} u_{n b}+b^{u}}{a_{i, j}^{u}}+d_{i, j}^{u}\left(p_{i, j}-p_{i+1, j}\right)
$$

Agora, defina a pseudo velocidade $\hat{u}_{i, j}$ como:

$$
\hat{u}_{i, j}=\frac{\sum a_{n b}^{u} u_{n b}+b^{u}}{a_{i, j}^{u}}
$$

É imediato notar que $\hat{u}_{i, j}$ é composto pelas velocidades nos nós vizinhos e não contém nenhum termo de pressão. Desta forma, a Eq. 3.36 se torna:

$$
u_{i, j}=\hat{u}_{i, j}+d_{i, j}^{u}\left(p_{i, j}-p_{i+1, j}\right)
$$

e, de forma similar,

$$
v_{i, j}=\hat{v}_{i, j}+d_{i, j}^{v}\left(p_{i, j}-p_{i, j+1}\right) .
$$

Percebe-se uma similaridade entre as duas últimas equações e as equações de correções definidas no final da seção anterior. Aqui, $\hat{u}$ e $\hat{v}$ aparecem no lugar de $u^{*}$ e $v^{*}$ e a pressão $p$ 
toma o lugar de $\delta p$. Consequentemente, substituindo as correções (Eqs. (3.38) e (3.39) na equação da continuidade discretizada (Eq. (3.8), temos uma nova equação para a pressão:

$$
a_{i, j}^{p} p_{i, j}=a_{i-1, j}^{p} p_{i-1, j}+a_{i+1, j}^{p} p_{i+1, j}+a_{i, j-1}^{p} p_{i, j-1}+a_{i, j+1}^{p} p_{i, j+1}+b^{p},
$$

com coeficientes como na Eq. (3.35) e

$$
b^{p}=\left(\hat{u}_{i, j}-\hat{u}_{i-1, j}\right) \delta y+\left(\hat{v}_{i, j}-\hat{v}_{i, j-1}\right) \delta x .
$$

Assim, podemos descrever o método SIMPLER (Alg. 2).

Algoritmo 2 SIMPLER (Semi-Implicit Method for Linked Pressure Equations Revised)

1. Comece com uma estimativa inicial para o campo de velocidades.

2. Calcule os coeficientes da equação do momentum e depois calcule $\hat{u}$ e $\hat{v}$ para as equações Eq. (3.37), substituindo os valores das velocidades vizinhas $u_{n b}$.

3. Calcule os coeficientes para a equação da pressão Eq. 3.40 e resolva-a para obter o campo de pressão.

4. Tratando o campo de pressão como $p^{*}$, resolva as equações do movimento para obter $u^{*} \mathrm{e}$ $v^{*}$.

5. Calcule a fonte de massa $b^{u}$, Eq. (3.35), e então resolva a equação de $\delta p$.

6. Faça a correção do campo de velocidade, usando as equações

$$
\begin{aligned}
& u^{n+1}=u^{*}+d^{u}\left(\delta p_{i}-\delta p_{i+1}\right) \\
& v^{n+1}=v^{*}+d^{v}\left(\delta p_{j}-\delta p_{j+1}\right),
\end{aligned}
$$

mas não faça a correção da pressão.

7. Retorne ao passo 2 e repita até a convergência, i.e., até que a equação discreta da continuidade seja satisfeita.

\subsection{Caso Teste: Escoamento em uma Cavidade}

Com o objetivo de testar nossa implementação do algoritmo SIMPLER, usaremos dados tabelados de soluções numéricas para o problema do escoamento em uma cavidade. Esse problema é conhecido na literatura como sendo um teste padrão para algoritmos de dinâmica dos fluidos computacional, por existirem diversos trabalhos com dados tabelados, como os apresentados nas Ref. [72] e Ref. [73]. 




Figura 3.5: Ilustração do problema do escoamento em uma cavidade.

Assim, iremos simular numericamente um escoamento bidimensional forçado pelo movimento da tampa de uma cavidade, através das equações do movimento:

$$
\begin{aligned}
\frac{\partial u}{\partial t}+\frac{\partial(u u)}{\partial x}+\frac{\partial(u v)}{\partial y} & =-\frac{\partial p}{\partial x}+\frac{1}{R e}\left(\frac{\partial^{2} u}{\partial x^{2}}+\frac{\partial^{2} u}{\partial y^{2}}\right) \\
\frac{\partial v}{\partial t}+\frac{\partial(u v)}{\partial x}+\frac{\partial(v v)}{\partial y} & =-\frac{\partial p}{\partial y}+\frac{1}{R e}\left(\frac{\partial^{2} v}{\partial x^{2}}+\frac{\partial^{2} v}{\partial y^{2}}\right) \\
\frac{\partial u}{\partial x}+\frac{\partial u}{\partial y} & =0
\end{aligned}
$$

sujeitas a algumas condições iniciais e de contorno que serão apresentadas adiante, e do algoritmo SIMPLER, descrito na seção 3.4. A constante $R e$ é conhecida como número de Reynolds:

$$
R e=\frac{\rho v}{\mu / L}=\frac{\text { forças inerciais }}{\text { forças viscosas }} \text {. }
$$

Este é um parâmetro adimensional que mede a razão entre as forças inerciais e as forças viscosas, mensurando a importância de cada uma dessas forças nas condições do escoamento. Aqui, $L$ é um comprimento característico no escoamento, $\mu$ a viscosidade e $\rho$ a densidade.

O problema é caracterizado da seguinte forma: a cavidade, de altura A e comprimento C, cujas paredes são sólidas e impermeáveis, tem seu interior totalmente preenchido com o fluido incompressível. Inicialmente, a tampa da cavidade e o fluido estão em repouso. No instante inicial $t_{o}$, ao acelerar instantaneamente a tampa da cavidade com uma velocidade 
$u_{o}$, damos origem ao escoamento. Neste problema, estamos considerando as velocidades $u$ e $v$ nulas no contorno da cavidade, que tem dimensões unitárias, $A=C=1$. Na tampa da cavidade, faremos $u_{o}=1$ e $v=0$, como podemos observar na Fig. 3.5. Além disso, iremos considerar o número de Reynolds como $R e=100$ para o nosso teste.

Todos os algoritmos necessários para a simulação em cavidade bidimensional foram implementados no MATLAB (MathWorks, Inc, Natick, MA, EUA) e executados em um computador com processador Intel Core i7 2,8 GHz com 16 GB de memória RAM utilizando o sistema operacional OSX Yosemite (Apple, Inc., Cupertino, CA, EUA). Buscou-se utilizar boas práticas de programação, relacionadas ao preenchimento e operações das matrizes esparsas envolvidas na solução numérica, com o intuito de acelerar os cálculos. Além disso, para realizar a solução dos sistemas lineares envolvidos foi utilizado o método do gradiente biconjugado estabilizado, nativo do MATLAB.

Na Fig. 3.6a temos a solução numérica no estado estacionário, i.e., $t \rightarrow \infty$ da velocidade $u$ no interior da cavidade quadrada de lado unitário, calculada com o software comercial ANSYS Fluent, usando uma malha $32 \times 32$ e $u_{o}=1$. A solução no estado estacionário é obtida após múltiplas iterações e é atingida na iteração $n_{0}$ (suficientemente grande) quando:

$$
\frac{\left\|u^{n_{0}+1}-u^{n_{0}}\right\|_{2}}{\delta t}<\varepsilon
$$

para $\varepsilon$ suficientemente pequeno. Na Fig. 3.6b, é apresentada uma comparação entre a solução obtida numericamente com o ANSYS Fluent com os valores tabelados no trabalho de Ghia et al. [72] no centro geométrico da cavidade na direção $x$. Os valores tabelados na Ref. [72] são uma fonte importante para comparação, pois os mesmos foram obtidos utilizando um algoritmo altamente eficiente e de alta resolução, para obtenção de soluções, exclusivamente, para o problema do escoamento em uma cavidade. Tal algoritmo é capaz também de obter soluções muito precisas mesmo para números de Reynolds muito baixos (que demandam malhas muito finas e passos de tempo muito pequenos). Dessa forma, é possível ver que os resultados obtidos utilizando o software ANSYS Fluent são qualitativamente e quantitativamente compatíveis com as soluções tabeladas.

Na Fig. 3.6c temos a solução numérica no estado estacionário da velocidade $u$ calculada usando o algoritmo SIMPLER implementado neste trabalho, utilizando uma malha $97 \times 97, \delta t=10^{-3}$ e $u_{o}=1$. Comparando as Fig. 3.6 a e Fig. 3.6 b, observa-se que, qualitati- 
(a)

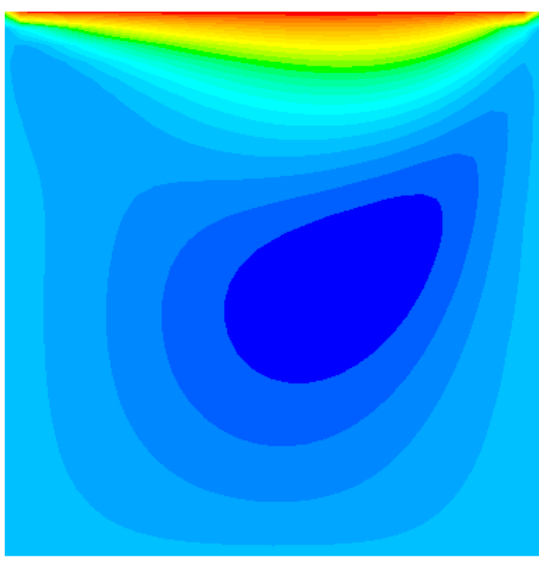

velocidade $u$

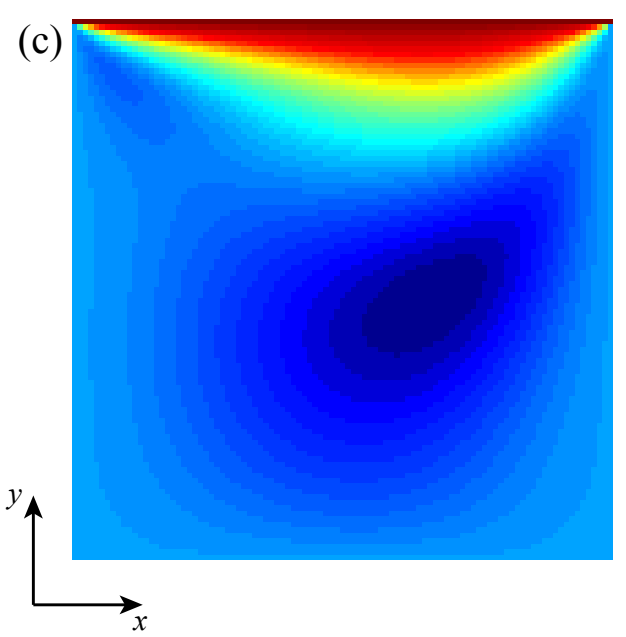

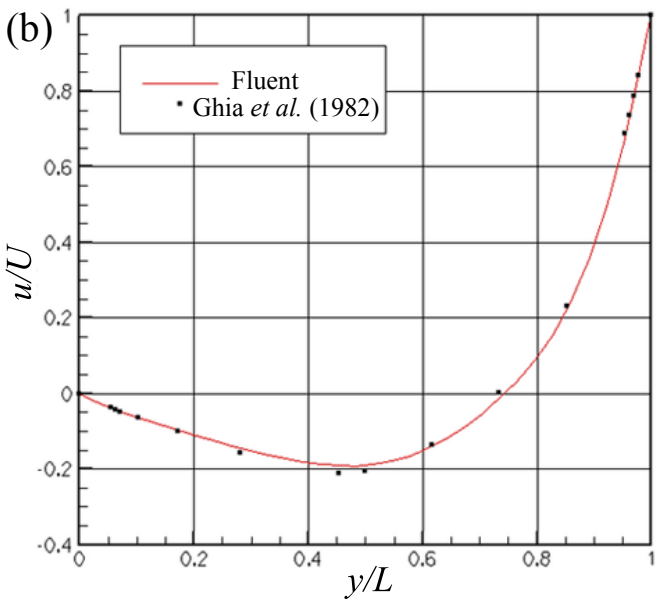

(d)

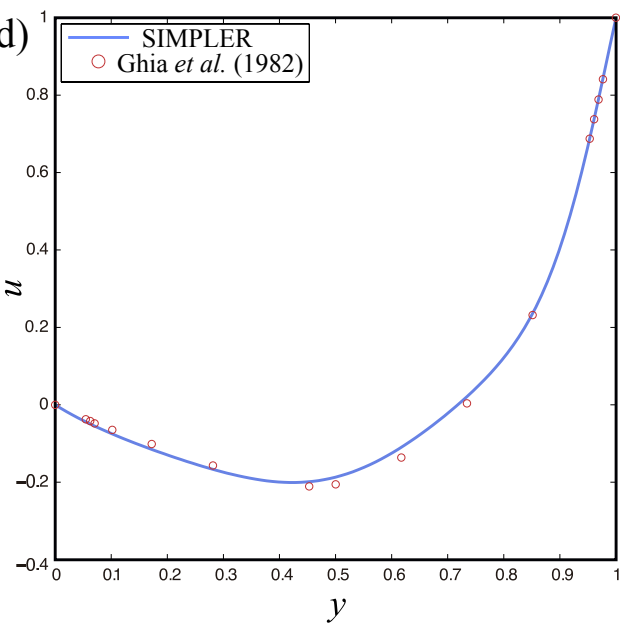

Figura 3.6: Solução para o escoamento em cavidade com $u_{o}=1$ e $R e=100$ : (a) obtida com software comercial ANSYS Fluent 6.1, com malha computacional de tamanho $32 \times 32$; e (c) obtida com algoritmo SIMPLER implementado neste trabalho, com malha computacional de tamanho $97 \times 97$. Comparação da velocidade $u$ obtida no centro geométrico da cavidade $(x=1 / 2)$ com os valores tabelados na Ref. [72]: (b) ANSYS Fluent; e (d) nossa implementação do SIMPLER.

vamente, a solução obtida com o algoritmo desenvolvido é compatível com a solução gerada pelo ANSYS Fluent. Aqui, foi escolhida uma malha mais fina que a utilizada no ANSYS Fluent e, também, um passo de tempo relativamente pequeno, pois o algoritmo desenvolvido não tem nenhum melhoramento com relação à resolução. Neste trabalho, optou-se por usar uma implementação mais simples, exatamente para facilitar a compreensão do método e mostrar que, mesmo com ela, é possível obter bons resultados. Na Fig. 3.6d, temos uma comparação entre a solução $u$ obtida numericamente no ponto $x=1 / 2$ e as soluções tabeladas na Ref. [72]. É importante ressaltar, novamente, que, tanto quantitativamente quanto qualitativamente, a solução obtida com o algoritmo desenvolvido está próxima da solução obtida com algoritmos comerciais e de alta resolução, bem como dos valores tabelados da literatura. 


\subsection{Conclusão}

Neste capítulo, foi apresentada uma dedução do algoritmo SIMPLER desenvolvido por Patankar \& Spalding [55] para as equações do movimento bidimensionais. Assim, uma versão bidimensional do algoritmo foi então implementada e um teste para um caso de escoamento bidimensional em uma cavidade foi realizado. Embora não mostrada aqui, uma versão tridimensional do algoritmo SIMPLER foi também implementada. Os resultados mostraram que a implementação do algoritmo neste trabalho é compatível com duas im-

plementações já consolidadas, de alto desempenho e alta resolução: o software comercial ANSYS Fluent 6.1 e os valores tabelados do escoamento em uma cavidade bidimensional por Ghia et al. [72]. 


\section{Capítulo 4}

\section{Simulações Computacionais do}

\section{Escoamento Sanguíneo Guiadas por}

\section{Dados de Contraste de Fase}

Neste capítulo, é descrito um método numérico para reconstruir escoamentos sanguíneos que são influenciados tanto por medições do escoamento usando ressonância magnética quanto por modelos matemáticos do escoamento dos fluidos. Dados medidos por contraste de fase são descritos como funções lineares das velocidades sobre a malha computacional e a dinâmica dos fluidos computacional controla a influência relativa das medidas diretas e das hipóteses do modelo. Essa abordagem foi brevemente descrita e resultados qualitativos preliminares foram apresentados por Nielsen \& Nayak [48]. Neste capítulo, é mostrado detalhadamente que o método combinado RM-DFC é capaz de produzir escoamentos que satisfazem as equações do movimento de fluidos, e que, por outro lado, tem uma concordância maior com as medidas por contraste de fase do que apenas as simulações núméricas dessas equações. Nielsen \& Nayak levaram em consideração apenas uma dimensão de velocidade medida por contraste de fase como influência para a solução combinada [4]. Dessa forma, é feito nesse capítulo uma extensão do método descrito por eles, agora, considerando as medidas por contraste de fase das velocidades nas três direções espaciais como influência para a solução combinada. Finalmente, são feitas análises qualitativas e quantitativas dos campos de velocidade simulados utilizando dados in vivo e de um phantom de escoamento pulsátil para validar o método. 
A lista de publicações relacionadas aos resultados apresentados neste capítulo se encontra no Anexo I.

\subsection{Métodos}

\subsubsection{Algoritmo SIMPLER}

As simulações do escoamento sanguíneo são feitas usando o algoritmo SIMPLER, desenvolvido por Patankar \& Spalding [47], e descrito com maiores detalhes no Capítulo 3. Esse procedimento é vastamente utilizado, tanto na área acadêmica quanto comercial, para determinar a solução numérica das equações do movimento da mecânica dos fluidos (Eqs. (3.2) e (3.3)).

O algoritmo SIMPLER começa com uma estimativa inicial do campo de velocidades e atualiza essa estimativa de forma iterativa. Em cada iteração, as equações do movimento são discretizadas e linearizadas usando as estimativas de campo de velocidade da iteração anterior (vide Seção 3.3).

Para uma malha computacional de tamanho $M \times N \times K$ no espaço tridimensional $x-y-z$, e considerando $L=M \cdot N \cdot K$, temos, como resultado da discretização das equações do movimento, na $n$-ésima iteração, três vetores coluna $\mathbf{u}_{n}, \mathbf{v}_{n}$ e $\mathbf{w}_{n}$, de tamanho $L \times 1$, correspondendo às velocidades nas direções $x, y$ e $z$, respectivamente. A construção do vetor $\mathbf{u}_{n}$ associado à uma iteração qualquer é ilustrado na Fig. 4.1. Os vetores $\mathbf{v}_{n}$ e $\mathbf{w}_{n}$ são construídos de maneira análoga, levando em consideração as componentes de velocidade nas direções $y$ e $z$, respectivamente.

É apresentado a seguir o desenvolvimento para a componente de velocidade na direção $x$, isto é, para $\mathbf{u}_{n}$. Para as demais componentes da velocidade nas direções $y$ e $z$ - isto é, $\mathbf{v}_{n}$ e $\mathbf{w}_{n}$, respectivamente - o desenvolvimento é idêntico, apenas substituindo $\mathbf{u}_{n}$, nas equações, por $\mathbf{v}_{n}$ ou $\mathbf{w}_{n}$. Note que, na $n$-ésima iteração, $\mathbf{u}_{n}, \mathbf{v}_{n}$ e $\mathbf{w}_{n}$ são calculados de forma independente. Assim, $\mathbf{v}_{n}$ e $\mathbf{w}_{n}$ não aparecem no cálculo de $\mathbf{u}_{n}$. Contudo, embora não explícito, o cálculo de $\mathbf{u}_{n}$ utiliza informações sobre as velocidades da iteração anterior em todas as três direções $\left(\mathbf{u}_{n-1}, \mathbf{v}_{n-1}\right.$ e $\left.\mathbf{w}_{n-1}\right)$, conforme detalhado no Capítulo 3.

Com respeito a $\mathbf{u}_{n}$, a discretização das equações do movimento, Eq. (3.2), resulta no 


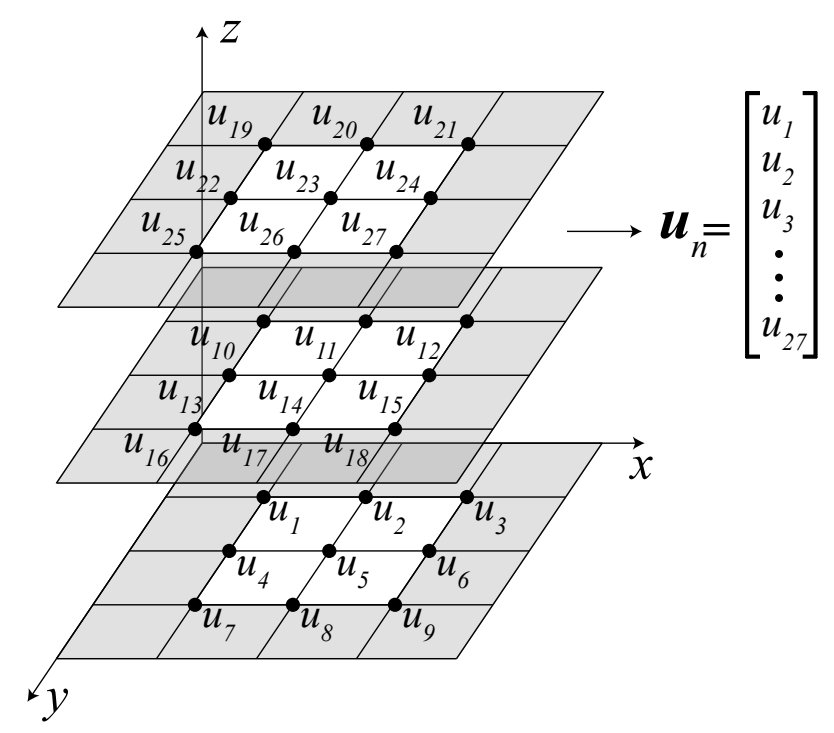

Figura 4.1: Construção do vetor $\mathbf{u}_{n}$ associado à uma iteração qualquer a partir dos valores de velocidade (componente na direção $x$ apenas) nos diversos pontos da grade tridimensional no espaço $x-y-z$. A velocidade em cada ponto em um espaço tridimensional com $M \times N \times K$ pontos é alocada como um coeficiente do vetor coluna $\mathbf{u}_{n}$, de tamanho $(M \cdot N \cdot K) \times 1$.

sistema linear quadrado:

$$
\mathbf{A}_{u, n-1} \mathbf{u}_{n}=\mathbf{b}_{u, n-1}
$$

em que $\mathbf{A}_{u, n-1}$ é uma matriz banda quadrada esparsa com sete diagonais com dimensão $L \times L$, que contém informações das três componentes de velocidade na iteração anterior $\left(\mathbf{u}_{n-1}, \mathbf{v}_{n-1}\right.$ e $\left.\mathbf{w}_{n-1}\right)$ e as constantes de densidade $(\rho)$ e viscosidade $(\mu)$; e $\mathbf{b}_{u, n-1}$ é um vetor coluna $L \times 1$ que contém informação não apenas sobre a densidade, a viscosidade e as três componentes de velocidade na iteração anterior, mas também sobre a pressão (calculada com base em $\mathbf{u}_{n-1}, \mathbf{v}_{n-1}$ e $\mathbf{w}_{n-1}$, de acordo com o Alg. 21. Por isso, a pressão não aparece explicitamente na Eq. 4.1.).

Tendo em vista que a discretização gera um sistema linear quadrado para cada componente de velocidade, temos que $\mathbf{u}_{n}$ é dado simplesmente por:

$$
\mathbf{u}_{n}=\left(\mathbf{A}_{u, n-1}\right)^{-1} \mathbf{b}_{u, n-1} .
$$

Desta forma, qualquer método iterativo para solução de sistemas lineares pode ser usado para determinar $\mathbf{u}_{n}$. Neste trabalho, é utilizado o método do gradiente biconjugado estabilizado.

Considerando $u_{n}, v_{n}$ e $w_{n}$ como sendo a forma Cartesiana dos vetores $\mathbf{u}_{n}, \mathbf{v}_{n}$ e $\mathbf{w}_{n}$, 
respectivamente, então para um dado instante de tempo $t=t_{0}$, o campo de velocidades $\vec{\nu}_{n}=u_{n} \mathbf{i}+v_{n} \mathbf{j}+w_{n} \mathbf{k}$ converge para a solução, neste instante de tempo, quando a equação da continuidade é satisfeita (passo 7 no Alg. 2). O tempo então é incrementado de uma quantidade $\delta t$, e o procedimento iterativo irá se repetir até obter a solução no instante de tempo $t=t_{0}+\delta t$, e assim por diante. Dessa forma, é posível determinar um campo de velocidades simulado que depende do tempo. Entretanto, seria necessário que o verdadeiro campo de velocidades no tempo $t=0$ fosse conhecido. Neste trabalho, o campo de velocidades exato é desconhecido, pois temos apenas um campo de velocidades com resolução espacial relativamente baixa e ruidoso. Por isso, calcularemos o campo de velocidades no estado estacionário:

$$
\vec{\nu}_{\infty}=\lim _{n \rightarrow \infty} \vec{\nu}_{n}
$$

sujeito às velocidades medidas com a ressonância magnética como condições de fronteira. Além disso, supondo que o campo de velocidades adquiridos através da RM em um dado instante de tempo representa o escoamento no estado estacionário, considerando o campo de velocidades medido naquele instante de tempo como sendo as condições de fronteira de entrada e saída do vaso [46, 32, 34].

Para cada fase cardíaca (um quadro temporal dentro do ciclo cardíaco), uma solução no estado estacionário $\vec{\nu}_{\infty}$ diferente é calculada, usando os dados de RM correspondentes àquele quadro temporal como condição de fronteira de entrada e saída para aquela fase. Nós obtemos cada um dos campos de velocidade $\vec{\nu}_{\infty}$ de forma independente para cada fase cardíaca, começando com uma estimativa inicial $\vec{\nu}_{0}$, e realizando as simulações, para frente no tempo, até a convergência, i.e.:

$$
\frac{\left\|\vec{\nu}_{n+1}-\vec{\nu}_{n}\right\|}{\delta t}<\varepsilon
$$

dado um $\varepsilon$ suficientemente pequeno $(\|\cdot\|$ representa a magnitude do vetor). Note que, aqui, o tempo $t$ é novamente um parâmetro de simulação apenas, e não está relacionado com os instantes de tempo ao longo do ciclo cardíaco.

\subsubsection{Incorporando as Medidas de RM ao SIMPLER}

A abordagem proposta é uma versão modificada do algoritmo SIMPLER, na qual linhas são adicionadas às matrizes $\mathbf{A}_{u}, \mathbf{A}_{v}$, e $\mathbf{A}_{w}$, e aos vetores $\boldsymbol{b}_{u}, \boldsymbol{b}_{v}$, e $\boldsymbol{b}_{w}$, com o objetivo 
de incorporar as medidas da ressonância magnética. Nesta abordagem, iremos supor que a velocidade medida com RM, em um dado voxel, pode ser expressa como uma combinação linear das velocidades na malha de cálculo computacional da DFC.

Na ausência de ruído, artefatos e distorções, o campo de velocidades medido com RM, $\vec{\nu}_{\mathrm{cf}}=\left(u_{\mathrm{cf}}, v_{\mathrm{cf}}, w_{\mathrm{cf}}\right)$, é uma versão borrada do campo de velocidades real, $\vec{\nu}=(u, v, w)$. Para a componente $u$, por exemplo, podemos escrever:

$$
u_{\mathrm{cf}}(x, y, z)=u(x, y, z) * \psi_{u}(x, y, z)
$$

em que * representa a convolução e $\psi_{u}(x, y, z)$ é a função de espalhamento de ponto associada à cobertura do espaço $k$ utilizada na aquisição de $u_{\text {cf }}$. De forma similar, as demais componentes são dadas por $v_{\mathrm{cf}}=v * \psi_{v}$ e $w_{\mathrm{cf}}=w * \psi_{w}$. Se todas as três componentes de velocidade são medidas usando a mesma cobertura do espaço $k$ (e, consequentemente, a mesma resolução espacial e campo de visão), então as funções de espalhamento de ponto são iguais, isto é, $\psi=\psi_{u}=\psi_{v}=\psi_{w}$. Além disso, para aquisições Cartesianas (TF3D) a função de espalhamento de ponto é dada por

$$
\psi(x, y, z)=\operatorname{sinc}\left(\frac{x}{\delta x}\right) \times \operatorname{sinc}\left(\frac{y}{\delta y}\right) \times \operatorname{sinc}\left(\frac{z}{\delta z}\right)
$$

e para aquisições em espiral

$$
\psi(x, y, z)=\operatorname{jinc}\left(\frac{\sqrt{x^{2}+y^{2}}}{\delta r}\right) \times \operatorname{sinc}\left(\frac{z}{\delta z}\right)
$$

em que $\delta x, \delta y, \delta z$ e $\delta r$ são as resoluções espaciais, conforme visto no Capítulo 2.

Se o campo de velocidades estimado através da DFC, $\vec{\nu}_{\infty}=\left(u_{\infty}, v_{\infty}, w_{\infty}\right)$, é uma representação precisa do verdadeiro campo de velocidades $\vec{\nu}$, então podemos escrever $u_{\mathrm{cf}}=$ $u_{\infty} * \psi_{u}, v_{\mathrm{cf}}=v_{\infty} * \psi_{v}$, e $w_{\mathrm{cf}}=w_{\infty} * \psi_{w}$. Assim, a discretização dessas equações irá resultar em três sistemas lineares. Usando a mesma notação introduzida anteriormente, para a n-ésima iteração do algoritmo da DFC, podemos escrever:

$$
\begin{aligned}
\boldsymbol{u}_{\mathrm{cf}} & =\mathbf{A}_{u, \mathrm{cf}} \boldsymbol{u}_{n} \\
\boldsymbol{v}_{\mathrm{cf}} & =\mathbf{A}_{v, \mathrm{cf}} \boldsymbol{v}_{n} \\
\boldsymbol{w}_{\mathrm{cf}} & =\mathbf{A}_{w, \mathrm{cf}} \boldsymbol{w}_{n}
\end{aligned}
$$


em que, $\boldsymbol{u}_{n}, \boldsymbol{v}_{n}$, e $\boldsymbol{w}_{n}$ são vetores coluna $L \times 1$ com as estimativas da DFC na $n$-ésima iteração, como definido anteriormente; $\boldsymbol{u}_{\mathrm{cf}}, \boldsymbol{v}_{\mathrm{cf}}$, e $\boldsymbol{w}_{\mathrm{cf}}$ são vetores coluna $Q \times 1$ com as medidas da ressonância magnética; e $\mathbf{A}_{u, c f}, \mathbf{A}_{v, \mathrm{cf}}$, e $\mathbf{A}_{w, \mathrm{cf}}$ são matrizes banda $L \times Q$ cujas entradas são calculadas a partir das funções de espalhamento de ponto $\psi_{u}, \psi_{v}$, e $\psi_{w}$, respectivamente. Novamente, se todas as velocidades medidas com a RM foram feitas com a mesma cobertura do espaço $k$ e reconstruídas em malhas idênticas, então $\mathbf{A}_{u, \mathrm{cf}}=\mathbf{A}_{v, \mathrm{cf}}=\mathbf{A}_{w, \mathrm{cf}}$.

Desta forma, uma vez que as Eqs. 4.1) e 4.8 estão na mesma malha computacional, podemos então escrever um sistema sobredeterminado para a componente $\mathbf{u}_{n}$ contendo informações sobre o modelo físico e a ressonância magnética:

$$
\left[\begin{array}{c}
\mathbf{A}_{u, n-1} \\
\mathbf{A}_{u, \mathrm{cf}}
\end{array}\right] \mathbf{u}_{n}=\left[\begin{array}{c}
\mathbf{b}_{u, n-1} \\
\mathbf{u}_{\mathrm{cf}}
\end{array}\right]
$$

Assim, fazendo:

$$
\boldsymbol{\Lambda}_{u, n-1}=\left[\begin{array}{c}
\mathbf{A}_{u, n-1} \\
\mathbf{A}_{u, \mathrm{cf}}
\end{array}\right] \text { e } \boldsymbol{\beta}_{u, n-1}=\left[\begin{array}{c}
\mathbf{b}_{u, n-1} \\
\mathbf{u}_{\mathrm{cf}}
\end{array}\right]
$$

temos que $\boldsymbol{\Lambda}_{u, n-1}$ é uma matriz $(L+Q) \times L$ e $\beta_{u, n-1}$ é um vetor coluna $(L+Q) \times 1$. Assim, para determinarmos a estimativa de velocidade, é necessário determinar a solução do sistema sobredeterminado $\boldsymbol{\Lambda}_{u, n-1} \mathbf{u}_{n}=\boldsymbol{\beta}_{u, n-1}$. Aqui, resolvemos o sistema dado pela Eq. (4.11) no sentido dos mínimos quadrados, i.e.:

$$
\mathbf{u}_{n}=\left\{\left(\boldsymbol{\Lambda}_{u, n-1}\right)^{T} \boldsymbol{\Lambda}_{u, n-1}\right\}^{-1}\left(\boldsymbol{\Lambda}_{u, n-1}\right)^{T} \boldsymbol{\beta}_{u, n-1}
$$

O campo de velocidades estimado pela simulação computacional guiada pela RM para cada uma das fases cardíacas é calculado independentemente, como a solução no estado estacionário $\vec{\nu}_{\infty}$, usando as velocidades de entrada e saída da artéria medidas pela RM para cada uma das fases cardíacas, como discutido anteriormente. Observe que a solução no estado estacionário é a solução mais próxima da RM, no sentido dos mínimos quadrados, que satisfaz tanto a equação do momento (Eq. 3.2. quanto a equação da continuidade (Eq. 3.3. Isto é garantido pelo fato que os sistemas sobredeterminados $\boldsymbol{\Lambda}_{u, n-1} \boldsymbol{u}_{n}=\boldsymbol{\beta}_{u, n-1}$, $\boldsymbol{\Lambda}_{v, n-1} \boldsymbol{v}_{n}=\boldsymbol{\beta}_{v, n-1}$, e $\boldsymbol{\Lambda}_{w, n-1} \boldsymbol{w}_{n}=\boldsymbol{\beta}_{w, n-1}$, são resolvidos a cada iteração do algoritmo SIMPLER (passos 2 e 4 do Alg. 2), e pelo critério de convergência (passo 7 do Alg. 2).

Se definirmos a resolução espacial da simulação computacional como a mesma da ve- 
locidade medida com RM, então as matrizes $\mathbf{A}_{u, c f}, \mathbf{A}_{v, c f}$, e $\mathbf{A}_{w, c f}$ serão matrizes identidade. Por outro lado, se usarmos uma resolução espacial mais fina para a simulação e reconstruirmos a velocidade medida $\vec{\nu}_{\text {cf }}$ nessa malha então as matrizes $\mathbf{A}_{u, \mathrm{cf}}, \mathbf{A}_{v, \mathrm{cf}}$, e $\mathbf{A}_{w, \mathrm{cf}}$ terão múltiplas diagonais e serão simétricas de tamanho $L \times L$. A diagonal principal terá apenas valores unitários e os demais dependerão do produto $\psi=\operatorname{sinc}(x / \delta x) \cdot \operatorname{sinc}(y / \delta y) \cdot \operatorname{sinc}(z / \delta z)$, dado pela função de espalhamento de ponto.

Quando apenas a velocidade medida na direção $z, \boldsymbol{w}_{\text {cf }}$, é usada para guiar as simulações computacionais, os vetores $\boldsymbol{u}_{\mathrm{cf}}$ e $\boldsymbol{v}_{\mathrm{cf}}$ serão nulos, e as matrizes $\mathbf{A}_{u, \mathrm{cf}}$ e $\mathbf{A}_{v, \mathrm{cf}}$ também. Neste caso, teremos $\boldsymbol{\Lambda}_{u, n-1}=\mathbf{A}_{u, n-1}, \boldsymbol{\Lambda}_{v, n-1}=\mathbf{A}_{v, n-1}, \boldsymbol{\beta}_{u, n-1}=\boldsymbol{b}_{u, n-1}$, e $\boldsymbol{\beta}_{v, n-1}=\boldsymbol{b}_{v, n-1}$. Portanto, $\boldsymbol{u}_{n}$ e $\boldsymbol{v}_{n}$ podem ser calculadas como $\boldsymbol{u}_{n}=\mathbf{A}_{u, n-1}^{-1} \boldsymbol{b}_{u, n-1}$ e $\boldsymbol{v}_{n}=\mathbf{A}_{v, n-1}^{-1} \boldsymbol{b}_{v, n-1}$, e, assim, esses sistemas deixam de ser sobredeterminados.

\subsection{Demonstração com Phantom}

\subsubsection{Aparato Experimental}

Os dados de contraste de fase utilizados para a demonstração com o phantom do método descrito neste capítulo foram adquiridos usando um phantom de escoamento pulsátil da carótida (Phantoms by Design, Inc., Bothell, WA) cedido pelo Professor Krishna S. Nayak, diretor do Laboratório de Engenharia em Ressonância Magnética da University of Southern California (Los Angeles, Califórnia, EUA). As aquisições foram realizadas durante o estágio de doutorado do autor, utilizando a máquina de ressonância magnética do Laboratório de Ressonância Magnética Funcional da University of Michigan (Ann Arbor, Michigan, EUA), sob a supervisão do Pesquisador Jon-Fredrik Nielsen, responsável, também, por desenvolver as sequências de pulso necessárias para a aquisição dos dados. O equipamento disponível para utilização no laboratório era um sistema GE Discovery 3T MR750.

Um phantom é um objeto desenvolvido especialmente para ser imageado dentro de um equipamento médico, que é utilizado para realizar diversos testes no sistema de imageamento. No caso da ressonância magnética, esses objetos são construídos de forma a possuirem grande magnetização e, consequentemente, proporcionarem um ótimo sinal. O phantom usado neste trabalho foi construído com o objetivo de gerar um escoamento pulsátil com frequência "cardíaca" constante, capaz de ser gatilhado e que produz escoamentos 
detectáveis pela máquina de ressonância magnética. Esse equipamento é composto de três partes fundamentais: o phantom (que é colocado dentro da máquina de RM) (Fig.4.2 a); um controlador que regula a frequência do escoamento e gera um sinal "cardíaco" para acionar a aquisição na frequência determinada (Fig. 4.2p); e um compressor de ar, fundamental para gerar o escoamento no interior do phantom (Fig. 4.2.). O fluido que está presente no interior dos tubos que compõem o phantom é um fluido mimetizador do sangue, tendo viscosidade e densidade similares a do sangue humano. Esses fluidos são normalmente compostos de uma mistura de polyvinylpyrrolidone, glicerina e água [74]. O escoamento no interior do phantom é produzido através de um impulso gerado pela movimentação de uma membrana de borracha, que, por sua vez, é devido a um pulso de ar comprimido produzido pelo compressor de ar. A frequência do escoamento pulsátil está relacionada à frequência de movimentação da membrana, que é regulada pelo controlador.

O controlador original do phantom apresentou defeito durante a aquisição dos dados e, dessa forma, foi necessária a confecção do controlador mostrado na Fig. 4.2b. Esse foi construído utilizando os seguintes materiais:

- 2 relés USB;

- 1 diodo limitador de corrente;

- 2 válvulas solenoidais pneumáticas de $12 \mathrm{~V}$;

- 1 fonte de $12 \mathrm{~V}$;

- 1 bateria de $9 \mathrm{~V}$;

- fios jumper;

- protoboard.

Um circuito com as válvulas solenoidais em paralelo, um diodo e um relé USB, então, foi montado na protoboard para efetuar o controle da frequência do escoamento pulsátil (Fig. 4.3). Para gerar o sinal "cardíaco" necessário para gatilhar a aquisição, foi montado um circuito em série composto de um relé USB e da bateria de $9 \mathrm{~V}$. O circuito era ligado a uma entrada auxiliar do equipamento de RM utilizada para gatilhos externos ao da máquina. Finalmente, o controle do bombeamento de ar comprimido para o phantom e o controle do gatilho foram feitos utilizando o MATLAB, por isso a utilização de relés USB. 


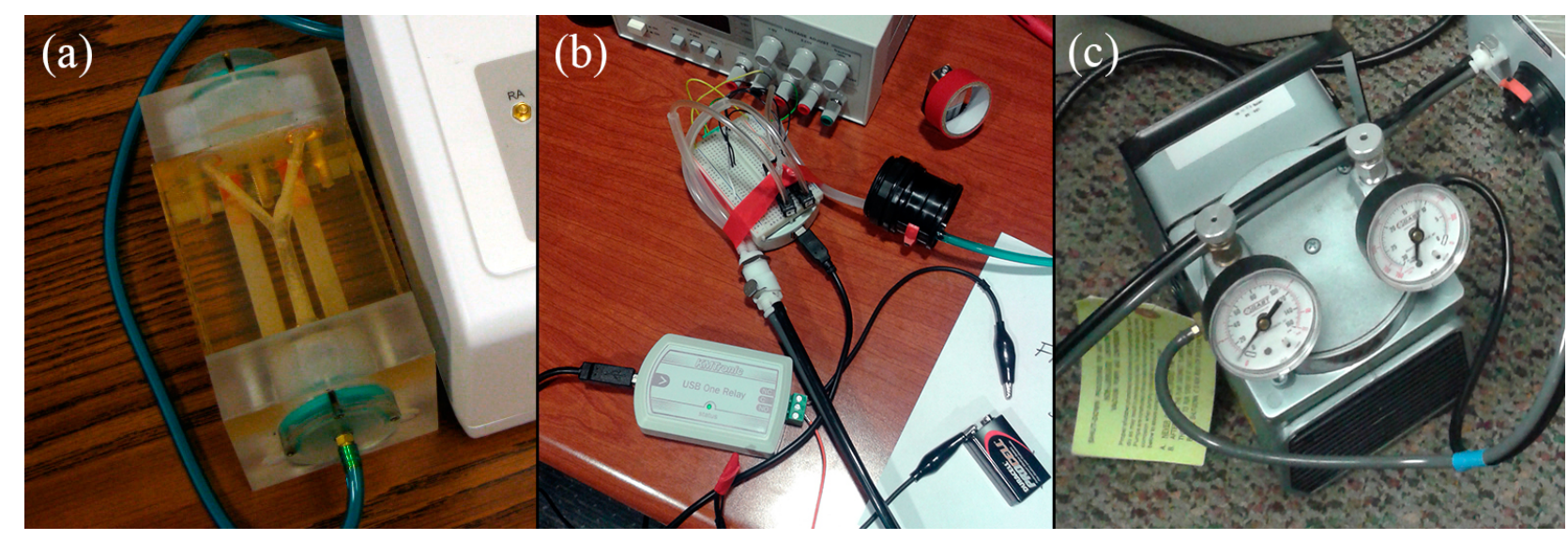

Figura 4.2: Componentes do phantom de escoamento pulsátil utilizado para aquisição dos dados de contraste de fase: (a) phantom da artéria carótida que contém em seu interior um fluido mimetizador do sangue; (b) controlador, produzido neste trabalho, que regula a frequência do escoamento e também fornece o sinal para aquisição gatilhada; (c) compressor de ar necessário para gerar o escoamento dentro do phantom.

Com isso, foram adquiridos dados de contraste de fase para o phantom com alta resolução espacial e também alta relação sinal-ruído. Os dados foram obtidos através de imagens volumétricas TF3D RF-spoiled gradient echo (SPGR) em oito instantes de tempo distintos do phantom de escoamento carotídeo pulsátil (tamanho do voxel $0,5 \times 0,5 \times$

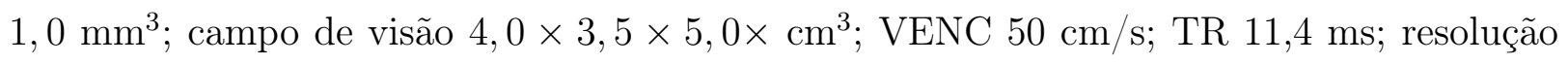
temporal 91,2 ms; ângulo de flip 8, 5; 40 minutos por aquisição; 9 NEX; frequência 60 bpm), em um sistema GE Discovery 3T MR750 (50 mT/m de amplitude máxima do gradiente e $200 \mathrm{mT} / \mathrm{m} / \mathrm{s}$ de taxa máxima de variação) com um arranjo de bobinas para cabeça de 32 canais (Nova Medical, Inc., Wilmington, MA, USA). Observa-se que a utilização de uma bobina de recepção para a carótida seria mais adequada para realizar as aquisições, pois proporcionaria uma relação sinal-ruído melhor e, consequentemente, seriam necessários menos NEX. No entanto, só estavam disponíveis bobinas de recepção para cabeça, tendo em vista que o laboratório em questão era um laboratório de ressonância magnética funcional. O eixo perpendicular ao plano de corte $(z)$ foi orientado na direção S/I. Cada um dos NEX foram adquiridos individualmente e as componentes de velocidade $u_{\mathrm{cf}}, v_{\mathrm{cf}}$ e $w_{\mathrm{cf}}$ foram reconstruídas usando os dados de todos os canais da bobina de recepção e fazendo a média de todos os 9 NEX. O "lúmen" foi segmentado manualmente limitando a fronteira do vaso em uma pilha de cortes axiais bidimensionais. 


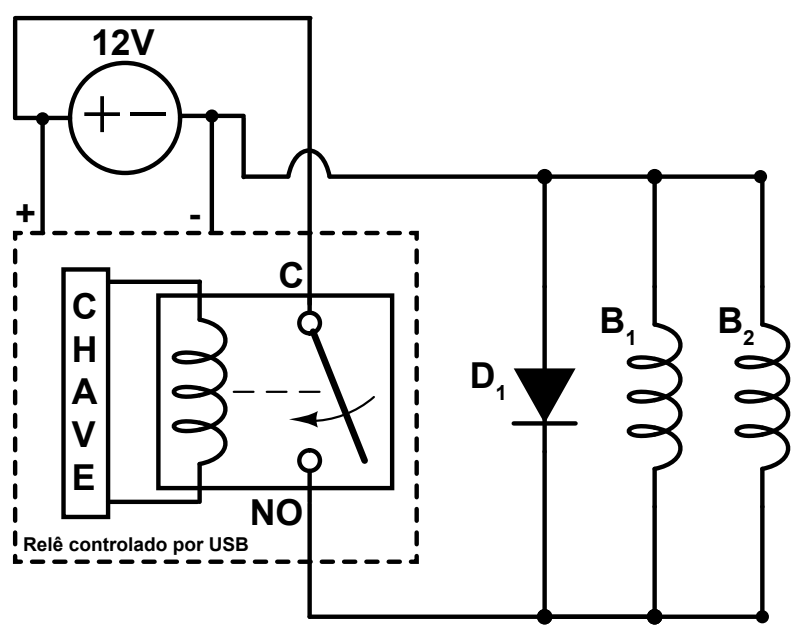

Figura 4.3: Esquema do controlador do phantom.

\subsubsection{Experimentos}

Utilizando os dados adquiridos com o phantom foram realizados dois experimentos. No primeiro, foram simulados por DFC três campos de velocidade: (i) uma solução numérica pura, que utilizava os dados do contraste de fase apenas como condições de contorno do tubo; (ii) uma solução numérica influenciada pela medida de velocidade $w_{\text {cf }}$ (que é a direção principal do escoamento); e (iii) uma solução numérica que incorporava as três direções de velocidade $u_{\mathrm{cf}}, v_{\mathrm{cf}}$ e $w_{\mathrm{cf}}$. Esses três campos de velocidade calculados foram comparados quantitativamente (utilizando a relação sinal-erro) e qualitativamente com as medidas originais do contraste de fase. No segundo experimento, adicionamos ruído Gaussiano às velocidades medidas com o contraste de fase e incorporamos as medidas ruidosas das três componentes de velocidade na solução numérica, utilizando o método proposto. Finalmente, calculamos a relação sinal-ruído tanto para a medida de contraste de fase ruidosa e a solução simulada, com o objetivo de verificar se o método proposto era capaz de reduzir ruído.

Para cada instante de tempo computacional $\delta t$, foi necessária apenas uma iteração para a convergência da equação da pressão, Eq. (3.32), com tempo de processamento de aproximadamente de 10 segundos por iteração. Para a simulação numérica pura, foram necessárias em média 90 iterações até a convergência; a simulação guiada com a velocidade $w_{\text {cf }}$ precisou em média de 40 iterações até a convergência; e, finalmente, a simulação guiada com as três componentes de velocidade necessitou em média de apenas 5 iterações, considerando $\varepsilon=10^{-3}$ na Eq. (4.4). 
Em ambos os experimentos, os valores de viscosidade e densidade do fluido contido no phantom, necessários para a simulação computacional, foram fornecidos pelo fabricante do phantom. Essas constantes são dadas por $\mu=0,005 \mathrm{~Pa} \cdot \mathrm{s}$ e $\rho=1100 \mathrm{~kg} / \mathrm{m}^{3}$, respectivamente. Nos experimentos com o phantom, as simulações computacionais foram realizadas com a mesma resolução espacial do campo de velocidades medido com RM, pois os dados adquiridos são de alta resolução espacial. Desta forma, as matrizes $\mathbf{A}_{u, c f}, \mathbf{A}_{v, \mathrm{cf}}$, e $\mathbf{A}_{w, \mathrm{cf}}$, definidas nas Eqs. 4.8)-4.10), são, nestes experimentos, matrizes identidade. Os cálculos do algoritmo consideraram a malha Cartesiana com resolução $0,5 \times 0,5 \times 1,0 \mathrm{~mm}^{3}$ e um passo de tempo computacional $\delta t=0,1 \mathrm{~ms}$. Aqui, o domínio de cálculo era retangular de tamanho $32,5 \times 9,0 \times 41,0 \mathrm{~mm}^{3}$. O procedimento para construção de soluções influenciadas tanto pela dinâmica dos fluidos computacional quanto pelos dados medidos por ressonância magnética foi repetido apenas para quatro dos oitos instantes de tempo medidos, fases 2 a 5. Isso se deve ao fato que não há movimento nas demais fases. Espera-se, neste caso, obter uma solução guiada mais próxima da medida com o contraste de fase do que solução obtida com as soluções puramente numéricas para as componentes de velocidade transversais $u$ e $v$.

Todos os algoritmos necessários foram implementados no MATLAB (MathWorks, Inc, Natick, MA, EUA) e executados em um computador com processador Intel Core i7 2,8 GHz com 16 GB de memória RAM utilizando o sistema operacional OSX Yosemite (Apple, Inc., Cupertino, CA, EUA). Buscou-se utilizar boas práticas de programação, relacionadas ao preenchimento e operações das matrizes esparsas envolvidas na solução numérica, com o intuito de agilizar os cálculos. Além disso, para realizar a solução dos sistemas lineares envolvidos foi utilizado o método do gradiente biconjugado estabilizado, nativo do MATLAB.

\subsubsection{Avaliação Quantitativa}

Para realizar uma comparação quantitativa entre o escoamento medido por contraste de fase e aqueles simulados usando a dinâmica dos fluidos computacional, está sendo calculada a relação sinal-erro (SER) entre cada campo de velocidades simulado e o campo de velocidades medido. A relação sinal-erro mede a relação entre a energia do sinal e a energia 
do erro de estimação:

$$
\mathrm{SER}=20 \log _{10}\left(\frac{R M S_{\text {sinal }}}{R M S_{\text {erro }}}\right)
$$

Consideramos o campo de velocidades medido com contraste de fase, $\vec{\nu}_{\mathrm{cf}}=\left(u_{\mathrm{cf}}, v_{\mathrm{cf}}, w_{\mathrm{cf}}\right)$, como o "sinal" de referência e consequentemente, o erro de estimação é o módulo da diferença vetorial entre o campo de velocidades estimado usando a DFC, $\vec{\nu}_{\infty}=\left(u_{\infty}, v_{\infty}, w_{\infty}\right)$, e o campo de velocidades de referência, $\vec{\nu}_{\mathrm{cf}}$. Dessa forma, mediremos a relação sinal-erro (em decibéis) como:

$$
\mathrm{SER}_{\vec{\nu}}=10 \log _{10}\left(\frac{\sum_{i, j, k}\left\|\vec{\nu}_{\mathrm{cf}}(i, j, k)\right\|_{2}^{2}}{\sum_{i, j, k}\left\|\vec{\nu}_{\infty}(i, j, k)-\vec{\nu}_{\mathrm{cf}}(i, j, k)\right\|_{2}^{2}}\right),
$$

em que os inteiros $i, j$ e $k$ representam os índices ao longo dos eixos $x, y$ e $z$, respectivamente, na malha computacional. De forma similar, a SER foi também calculada individualmente para cada componente de velocidade, como:

$$
\begin{aligned}
\operatorname{SER}_{u} & =10 \log _{10}\left(\frac{\sum_{i, j, k} u_{\mathrm{cf}}(i, j, k)^{2}}{\sum_{i, j, k}\left[u_{\infty}(i, j, k)-u_{\mathrm{cf}}(i, j, k)\right]^{2}}\right) \\
\mathrm{SER}_{v} & =10 \log _{10}\left(\frac{\sum_{i, j, k} v_{\mathrm{cf}}(i, j, k)^{2}}{\sum_{i, j, k}\left[v_{\infty}(i, j, k)-v_{\mathrm{cf}}(i, j, k)\right]^{2}}\right) \\
\mathrm{SER}_{w} & =10 \log _{10}\left(\frac{\sum_{i, j, k} w_{\mathrm{cf}}(i, j, k)^{2}}{\sum_{i, j, k}\left[w_{\infty}(i, j, k)-w_{\mathrm{cf}}(i, j, k)\right]^{2}}\right) .
\end{aligned}
$$

Através das Eqs. 4.15 a 4.18 foram calculadas então a SER para cada um dos quatro quadros temporais considerados. Além disso, é imediato notar da Eqs. 4.15) a 4.18) que, quanto menor for a diferença entre o conjunto de dados simulado e o conjunto de dados medido, maior será a SER.

\subsubsection{Avaliação da Redução de Ruído}

Finalmente, com o objetivo de verificar o efeito de redução de ruído do método aqui desenvolvido, adicionamos ruído Gaussiano de média nula e desvio padrão $\sigma$ às componentes de velocidade medidas por contraste de fase na fase 4 do phantom, e em seguida, as três componentes de velocidade ruidosas foram utilizadas para guiar a simulação numérica. Essa fase foi escolhida por estar próxima ao pico de velocidade do escoamento. Esse procedimento foi repetido para três valores diferentes do desvio padrão. Assim, a cada uma 
das componentes de velocidade - $u_{\mathrm{cf}}, v_{\mathrm{cf}}$ e $w_{\mathrm{cf}}$ - foi adicionado ruído Gaussiano de média nula e desvio padrão $\sigma=7 \mathrm{~cm} / \mathrm{s}, \sigma=8 \mathrm{~cm} / \mathrm{s}$ e $\sigma=9 \mathrm{~cm} / \mathrm{s}$.

\subsubsection{Resultados}

Nas Figuras 4.4, 4.5 e 4.6 são mostradas comparações qualitativas das componentes de velocidade $u_{\mathrm{cf}}, v_{\mathrm{cf}}$ e $w_{\mathrm{cf}}$, medidas usando a ressonância magnética, para três cortes transversais da artéria carótida simulada do phantom, nas quatro diferentes fases citadas, entre: as soluções numéricas produzidas com influência do contraste de fase apenas como condição de fronteira de entrada e saída, com a influência da velocidade medida, na direção $z$ (wcf) e a solução guiada pelas medições de velocidade por contraste de fase nas três direções $x, y$ e $z$.

Na Figura 4.7 é apresentado o divergente do campo de velocidades para a medida por contraste de fase e também das três soluções numéricas produzidas. É possível observar de forma imediata na Fig. 4.7 que o campo de velocidades medido por contraste de fase não satisfaz a equação da continuidade, Eq. (3.8). Um campo de velocidade não satisfazer a equação da continuidade significa que naquele escoamento a massa não é conservada ou que o escoamento é compressível. Nenhuma dessas duas possibilidades é cabível para o escoamento medido para o phantom. Primeiramente, o fluido mimetizador do sangue é incompressível por ser composto basicamente de água e glicerina. Além disso, no interior do tubo onde ocorre o escoamento não há fontes de fluido ou vazamentos, dessa forma, não é possível que o escoamento não tenha sua massa conservada. Assim, este é um fenômemo que possivelmente está relacionado as diversas fontes de erro presentes na medição por ressonância magnética, como, por exemplo, ruídos, efeitos de volume parcial e não homogeneidade do campo magnético $B_{0}[75]$.

Os resultados apresentados nas Figs. 4.4 a 4.7 mostram que a utilização dos campos de velocidade medidos por contraste de fase para guiar as soluções computacionais leva a soluções que se assemelham qualitativamente entre si, mas que, ao mesmo tempo, satisfazem a equação da continuidade $(\nabla \cdot \vec{\nu}=0)$. Isso é considerado como um procedimento de redução de ruído e erro, tendo em vista que o campo de velocidades medido por contraste de fase deveria satisfazer naturalmente a equação da continuidade. É importante observar que, na literatura, existem diferentes métodos de pré, ou, pós-processamento aplicáveis a um de 
campo de velocidades medido por contraste de fase com o objetivo de fazê-lo satisfazer a equação da continuidade [42, 43].

Notamos que a solução numérica pura, cuja única influência das medidas de RM está nas condições de fronteira de entrada e saída, produz soluções que satisfazem o modelo físico, mas qualitativamente são sensivelmente diferentes das medidas reais. Também é possível perceber, em uma comparação entre as Figs. 4.4 e 4.5, que a utilização da componente de velocidade na direção principal do escoamento (eixo $z$ ) produz uma solução que satisfaz o modelo físico, mas tendo em vista que as componentes de velocidade $u$ e $v$ no plano $x-y$ são obtidos apenas com a simulação numérica, elas são qualitativamente diferentes das componentes medidas com o contraste de fase. Isso também ocorre com a solução combinada guiada pelas componentes de velocidade nas três direções; no entanto, é possível ver claramente que essas solução estão mais próximas das medidas de RM do que solução combinada usando apenas a componente de velocidade medida na direção $z$.

Na Fig. 4.8 são apresentados, para os quatro quadros temporais considerados, os campos de velocidade do escoamento medido por contraste de fase, bem como dos escoamentos obtidos por meio das simulações numéricas (guiadas ou não). Em uma comparação qualitativa entre a medida do contraste de fase e os campos simulados, a solução que apresenta uma maior similaridade com o escoamento medido é, mais uma vez, a simulação guiada pelas três componentes de velocidade da ressonância magnética. Percebe-se ainda que há uma semelhança qualitativa grande entre a simulação numérica que leva em consideração somente a componente de velocidade $w_{\text {cf }}$ como guia e aquela que considera as direções $x, y$ e $z$ da velocidade como guia.

Nas Tabelas 4.1, 4.2, 4.3 e 4.4 são apresentados os resultados do cálculo da relação sinal-erro para as soluções numéricas realizadas. Percebe-se de imediato que, nos quatro quadros temporais considerados, o valor da SER para as componentes de velocidade que se encontram no plano $x-y$ é significativamente menor que a SER da componente na direção z. Isso pode ser explicado pelo fato do mesmo VENC ter sido utilizado para as três componentes de velocidade, mas o movimento do fluido ocorre predominantemente na direção $z$, ou seja, esta é a dimensão onde a componente de velocidade possui o maior módulo, e, consequentemente, é a direção que possui mais sinal. Por exemplo, para a fase 4, o sinal da

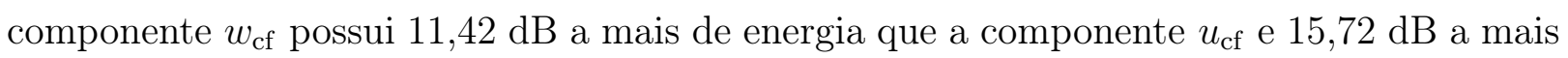
de energia que a componente $v_{\text {cf }}$. Também é notável que, nas quatro fases consideradas, a 
componente de velocidade na direção $z$ da solução numérica que considera apenas a componente $w_{\text {cf }}$ como guia é maior do que a da solução numérica pura e também da solução numérica guiada por todas as três dimensões de velocidade. Esse é um comportamento esperado, pois estando a velocidade na dimensão $z$ atada àquela medida por RM, então é permitido às componentes de velocidade do plano $x-y$ variarem livremente. É possível observar qualitativamente nas Figs. 4.4, 4.5 e 4.6 que a solução computacional que se assemelha mais com os dados do contraste de fase é aquela que é guiada pelas três componentes de velocidade. Esse comportamento é demonstrado também de forma quantitativa para as quatro fases consideradas, verificando que há um ganho maior de SER com relação ao contraste de fase comparado à simulação numérica pura e também àquela que é guiada apenas pela componente $w_{\text {cf }}$. Finalmente, percebe-se que, em geral, a simulação que possui a melhor SER para o campo de velocidades é aquela que se utiliza das três componentes de velocidade medidas por contraste de fase como guia, sendo que para as fases $2,3,4$ e 5 há um ganho de $7,15 \mathrm{~dB}, 7,06 \mathrm{~dB}, 6,56 \mathrm{~dB}$ e $7,38 \mathrm{~dB}$, respectivamente.

As Figs. 4.9, 4.10 e 4.11 mostram os resultados do segundo experimento, o qual tinha como objetivo verificar o efeito de redução de ruído do método aqui desenvolvido. É apresentada uma comparação entre as componentes do contraste de fase sem ruído adicionado (Figs. 4.9a, 4.10a e 4.11a), com ruído Gaussiano adicionado (Figs. 4.9b, 4.10b e 4.11b) e as componentes do campo de velocidade simulado por DFC guiado pelas três componentes (ruidosas) de velocidade (Figs. 4.9c, 4.10k e 4.11c). Qualitativamente, é possível apreciar uma pequena redução nos níveis de ruídos quando se compara as componentes de velocidade do contraste de fase com ruído adicionado (Figs. 4.9p, 4.10 b e 4.11b; e Figs. 4.9d, $4.10 \mathrm{~d}$ e $4.11 \mathrm{~d})$ com os resultados obtidos através da simulação computacional guiada pelas três componentes ruidosas de velocidade (Figs. 4.9k, 4.10k e 4.11k; e Figs. 4.9k, 4.10k e 4.11 ). Por exemplo, para a componente de velocidade $u$, as médias do valor absoluto do erro para cada um dos diferentes valores do desvio padrão $(\sigma=7,8$ e $9 \mathrm{~cm} / \mathrm{s})$ são dadas por $4,5 \mathrm{~cm} / \mathrm{s}, 4,6 \mathrm{~cm} / \mathrm{s}$ e $5,0 \mathrm{~cm} / \mathrm{s}$, respectivamente. Finalmente, a Tabela 4.5 apresenta uma análise quantitativa desse experimento. É apresentada a SER dos campos de velocidade medidos por contraste de fase com ruído, bem como a SER dos campos obtidos por meio da DFC guiada pelas três componentes de velocidade medidas por RM (ambos com relação aos campos de velocidade medidos por contraste de fase, sem adição de ruído). Nos três níveis de ruído adicionado, verificou-se um ganho na SER tanto para o vetor de velocidade 
$(\vec{\nu})$, quanto para cada uma das componentes individualmente. Houve um ganho de 1,00 dB, $1,23 \mathrm{~dB}$ e 1,51 dB na SER do vetor de velocidade nos experimentos com $\sigma=7,8$ e $9 \mathrm{~cm} / \mathrm{s}$, respectivamente. Este resultado sugere que o método proposto neste trabalho pode também ser utilizado como uma técnica de redução de ruído para medidas de velocidade por RM. 


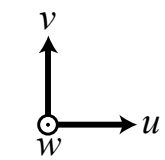

(a)

a)

$\mathrm{CF}$

DFC
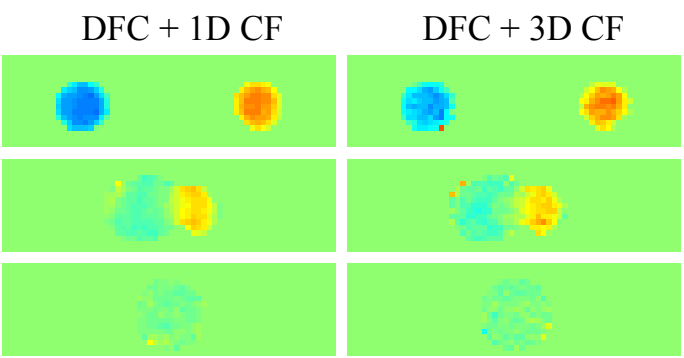

(b)
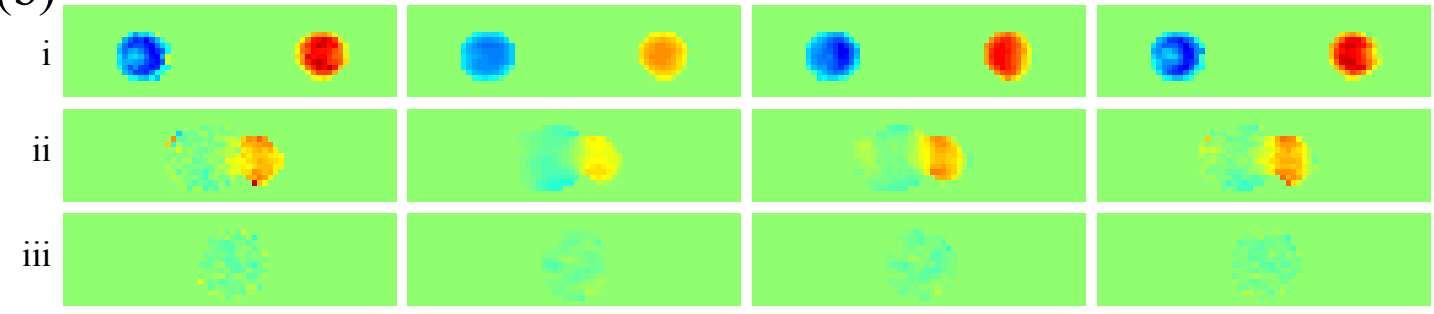

(c)
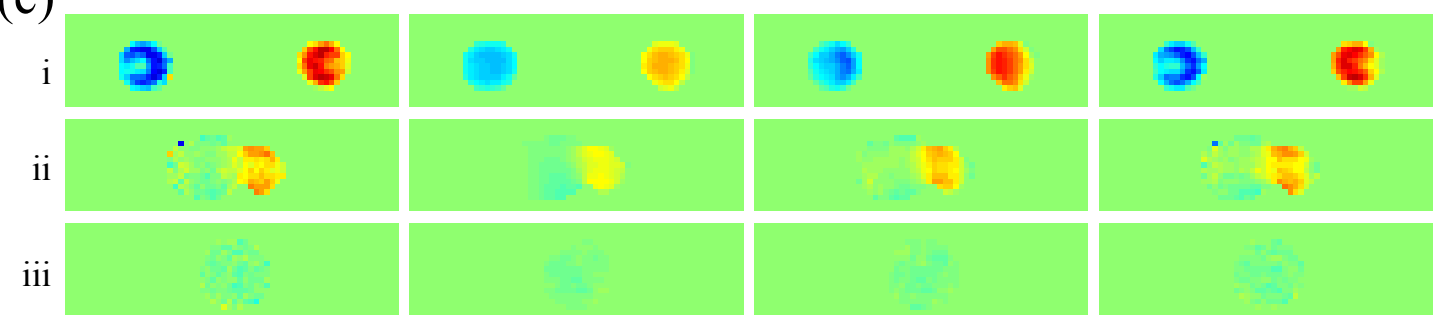

(d)
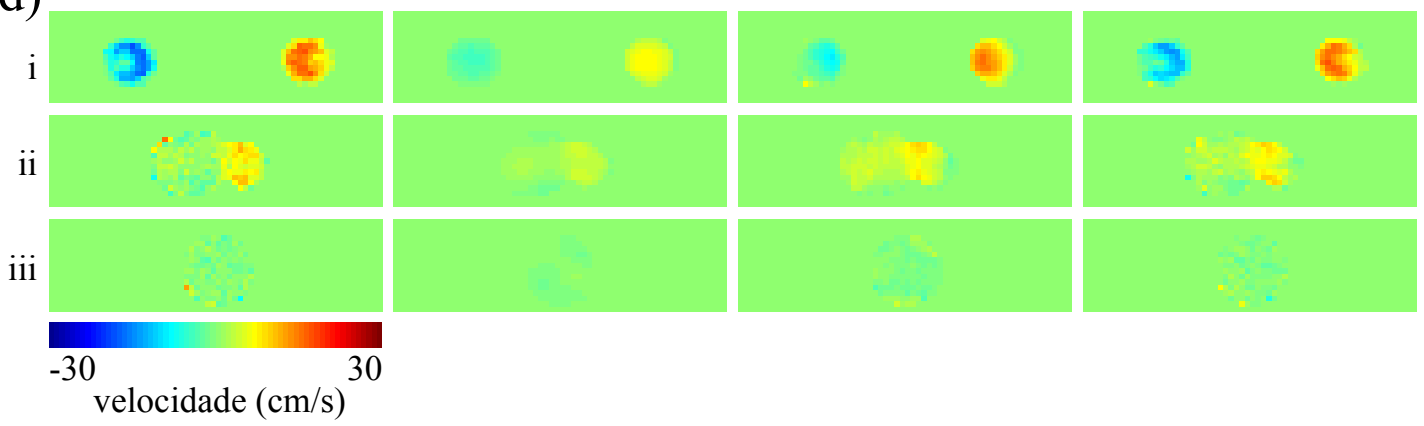

velocidade $(\mathrm{cm} / \mathrm{s})$

Figura 4.4: Seções transversais da componente de velocidade na direção $u$, em quatro instantes de tempo diferentes: (a) fase 2; (b) fase 3; (c) fase 4; (d) fase 5. Na figura, são mostrados três diferentes cortes da "artéria carótida" do phantom, a saber: (iii) carótida comum, (ii) bifurcação da carótida e (i) carótida bifurcada. Cada coluna representa um dos diferentes métodos de estimação: contraste de fase $(\mathrm{CF})$, simulação numérica pura (DFC), simulação numérica guiada pela componente de velocidade do contraste de fase na direção $z(\mathrm{DFC}+1 \mathrm{D} \mathrm{CF})$ e solução numérica guiada pelas três componentes de velocidade do contraste de fase (DFC $+3 \mathrm{D} \mathrm{CF})$. 


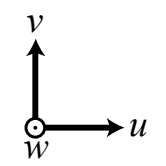

(a)
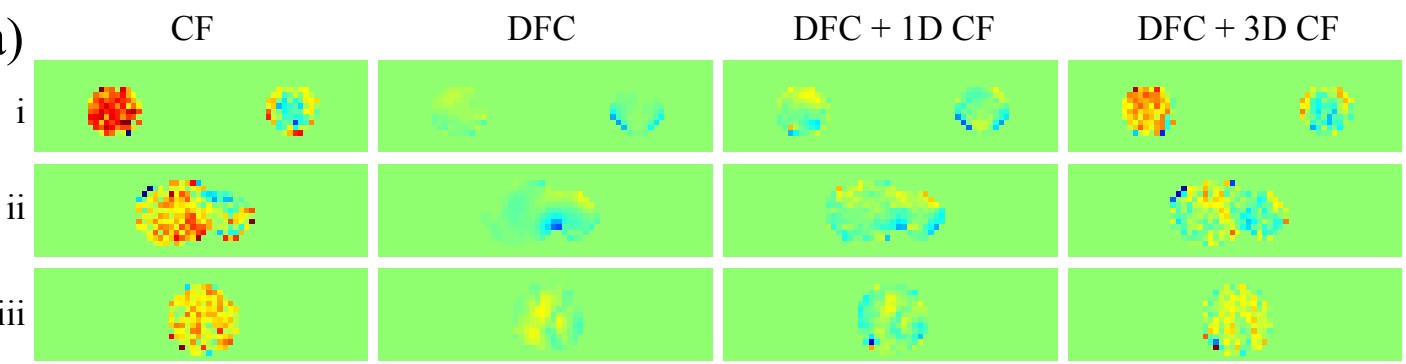

(b)
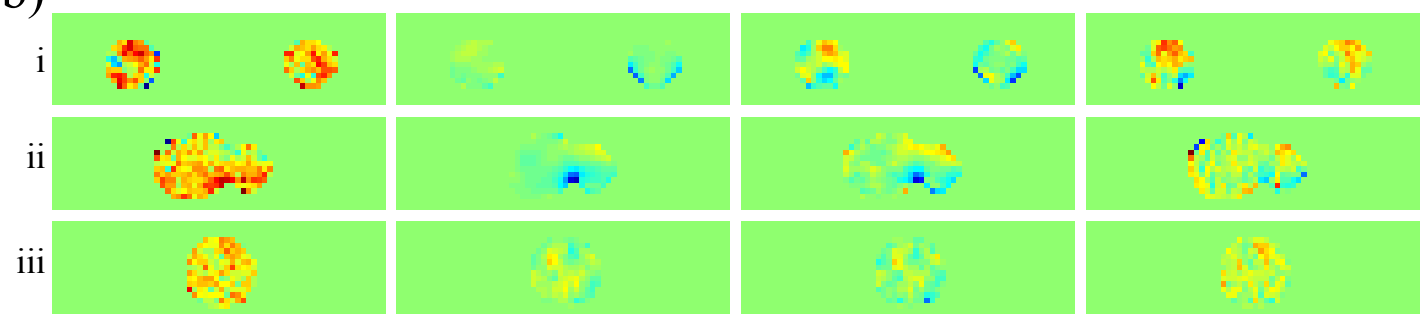

(c)
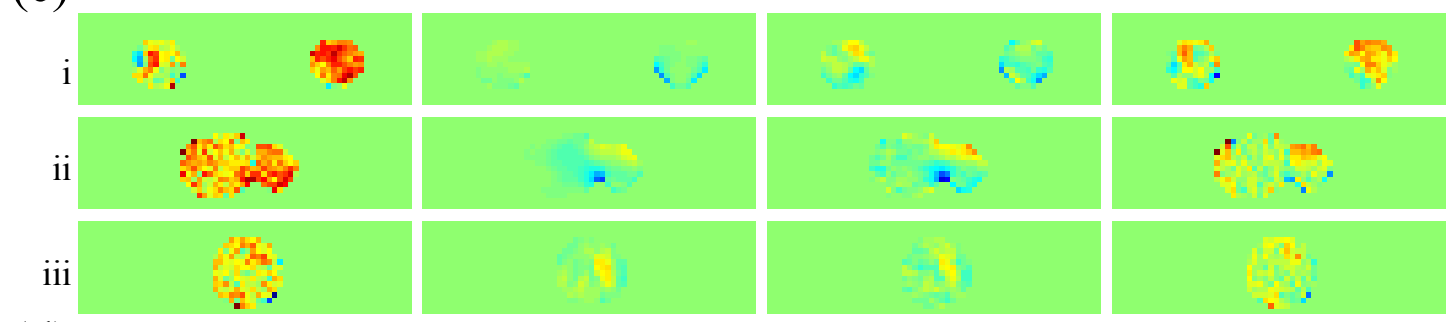

(d)
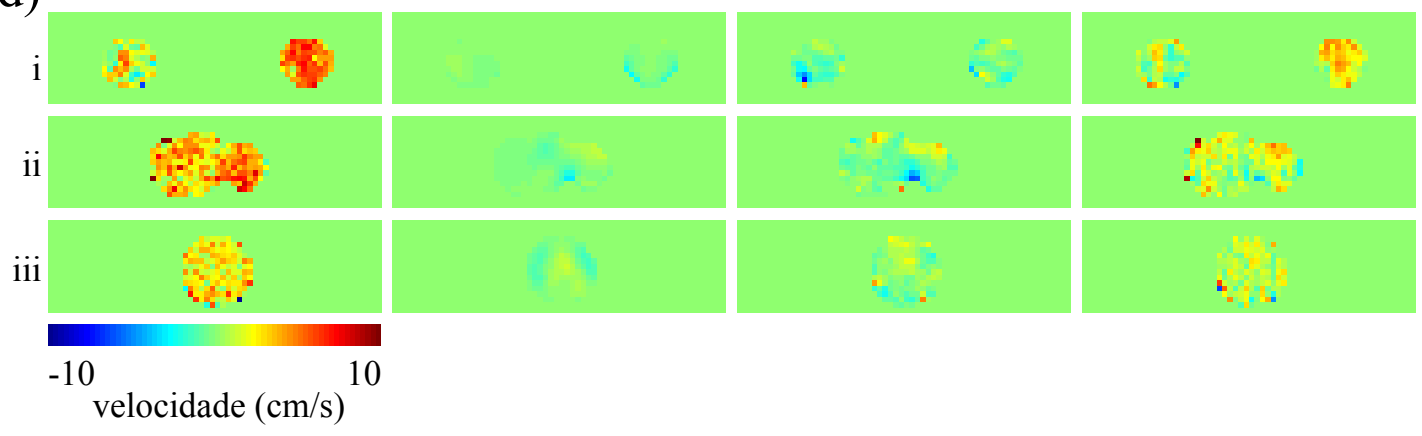

Figura 4.5: Seções transversais da componente de velocidade na direção $v$, em quatro instantes de tempo diferentes: (a) fase 2; (b) fase 3; (c) fase 4; (d) fase 5. Na figura, são mostrados três diferentes cortes da "artéria carótida" do phantom, a saber: (iii) carótida comum, (ii) bifurcação da carótida e (i) carótida bifurcada. Cada coluna representa um dos diferentes métodos de estimação: contraste de fase $(\mathrm{CF})$, simulação numérica pura (DFC), simulação numérica guiada pela componente de velocidade do contraste de fase na direção $z(\mathrm{DFC}+1 \mathrm{D} \mathrm{CF})$ e solução numérica guiada pelas três componentes de velocidade do contraste de fase (DFC $+3 \mathrm{D} \mathrm{CF})$. 


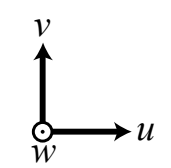

(a)

a) $\quad \mathrm{CF}$

$\mathrm{CF} \quad \mathrm{DFC}$

$\mathrm{DFC}+1 \mathrm{D} \mathrm{CF}$

$\mathrm{DFC}+3 \mathrm{D} \mathrm{CF}$
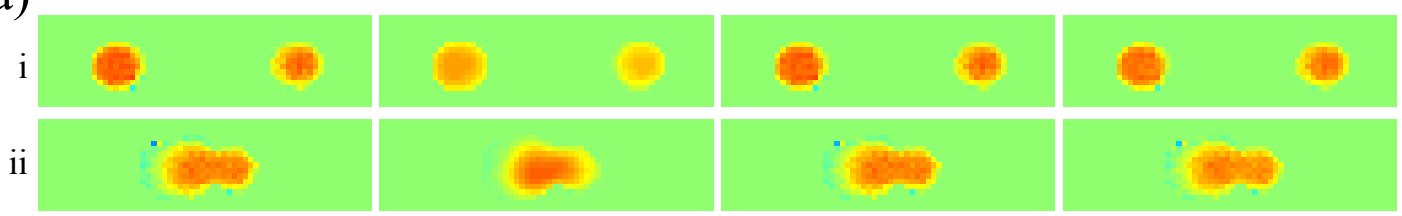

iii


(b)
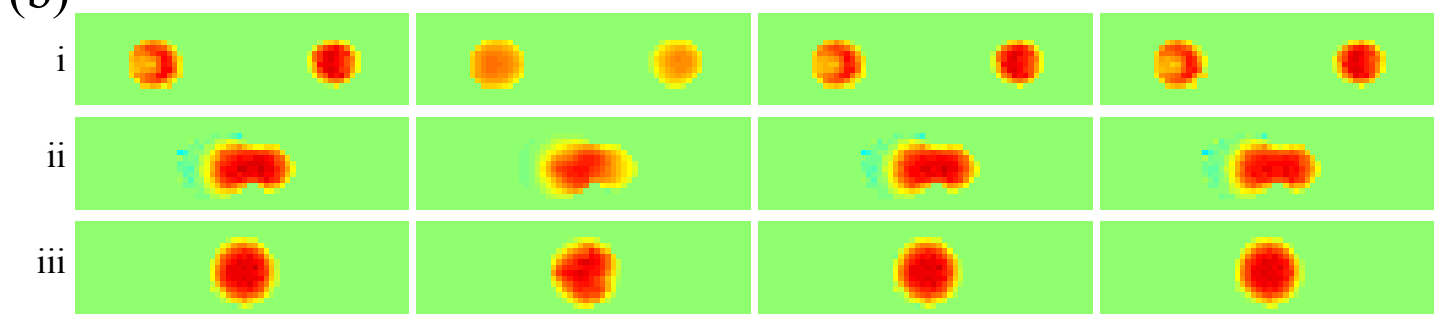

(c)
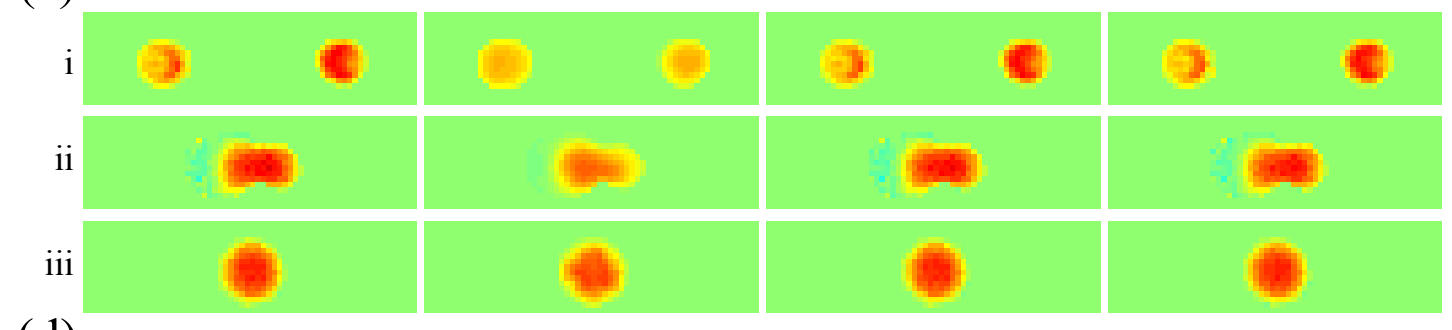

(d)
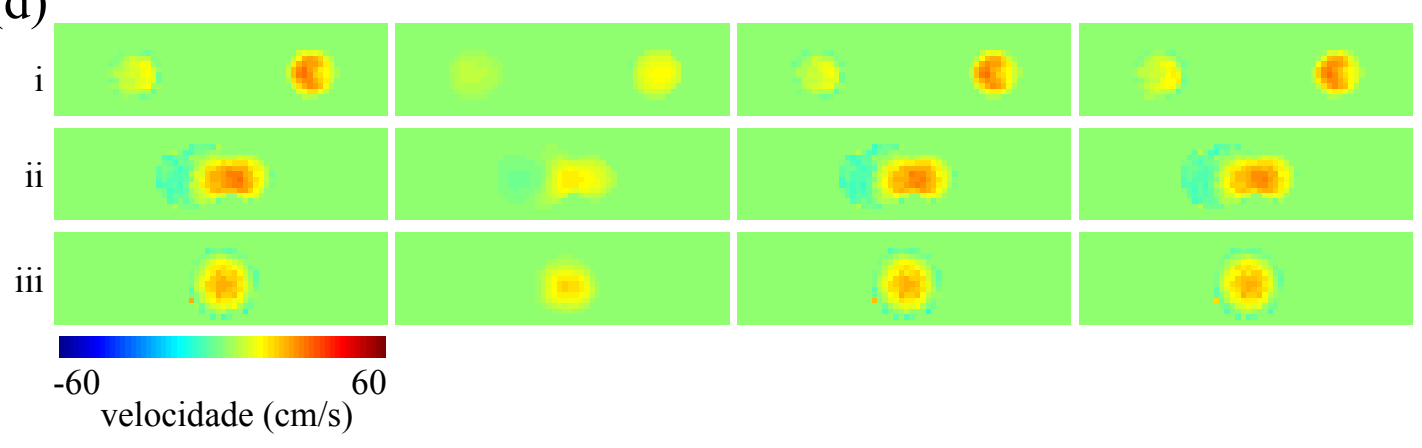

velocidade $(\mathrm{cm} / \mathrm{s})$

Figura 4.6: Seções transversais da componente de velocidade na direção $w$, em quatro instantes de tempo diferentes: (a) fase 2; (b) fase 3; (c) fase 4; (d) fase 5. Na figura, são mostrados três diferentes cortes da "artéria carótida" do phantom, a saber: (iii) carótida comum, (ii) bifurcação da carótida e (i) carótida bifurcada. Cada coluna representa um dos diferentes métodos de estimação: contraste de fase $(\mathrm{CF})$, simulação numérica pura (DFC), simulação numérica guiada pela componente de velocidade do contraste de fase na direção $z(\mathrm{DFC}+1 \mathrm{D} \mathrm{CF})$ e solução numérica guiada pelas três componentes de velocidade do contraste de fase $(\mathrm{DFC}+3 \mathrm{D} \mathrm{CF})$. 


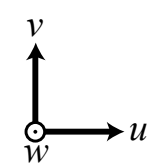

(a)

a) $\quad \mathrm{CF}$

DFC

$\mathrm{DFC}+1 \mathrm{D} \mathrm{CF}$

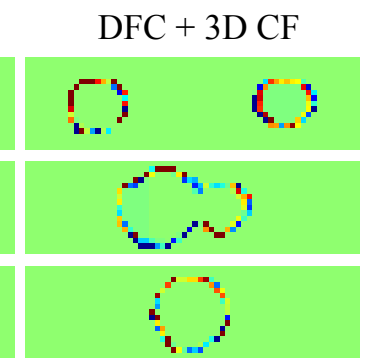

(b)
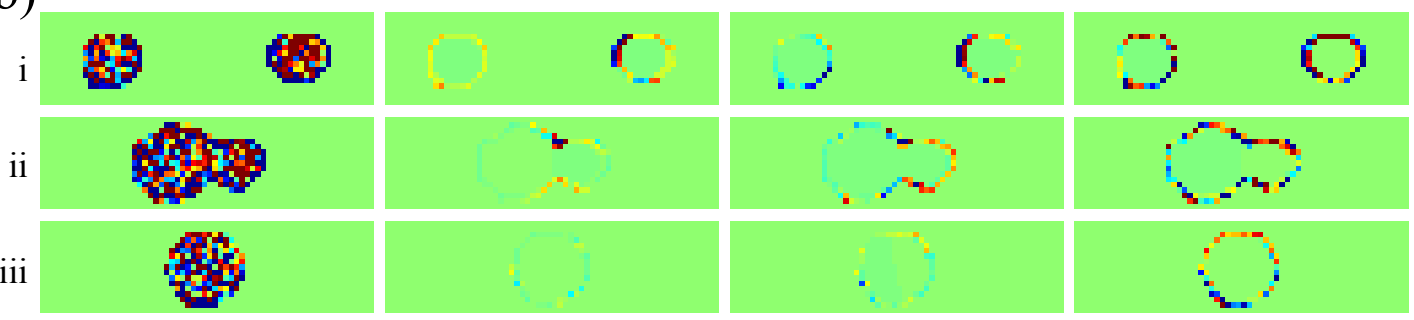

(c)
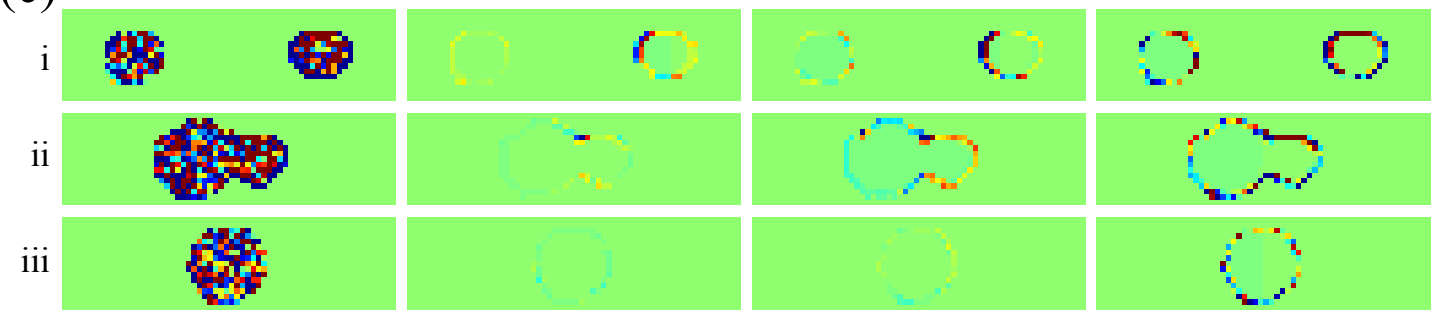

(d)
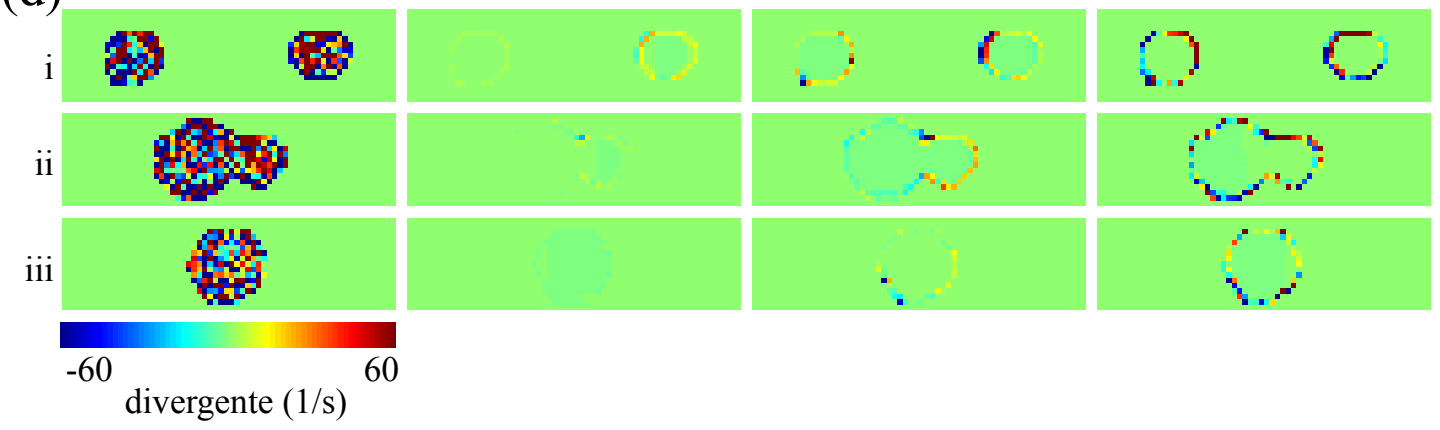

Figura 4.7: Seções transversais do $\operatorname{div}(\vec{\nu})$ em quatro instantes de tempo diferentes: (a) fase 2; (b) fase 3; (c) fase 4; (d) fase 5. Na figura, as linhas representam três diferentes cortes da artéria carótida simulada do phantom, a saber: (iii) carótida comum, (ii) bifurcação da carótida e (i) carótida bifurcada; e as colunas representam os diferentes métodos de aquisição: contraste de fase $(\mathrm{CF})$, simulação numérica pura (DFC), simulação numérica guiada pela componente de velocidade do contraste de fase na direção $z(\mathrm{DFC}+1 \mathrm{D} \mathrm{CF})$ e solução numérica guiada pelas três componentes de velocidade do contraste de fase (DFC $+3 \mathrm{D} C F)$. 
(a)


(b)
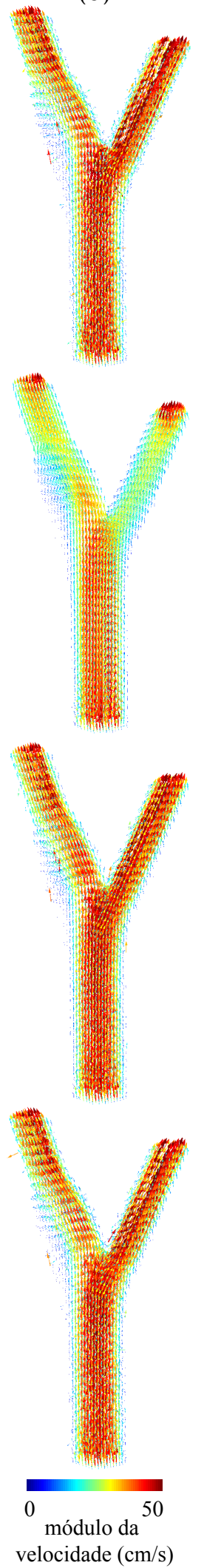

(c)
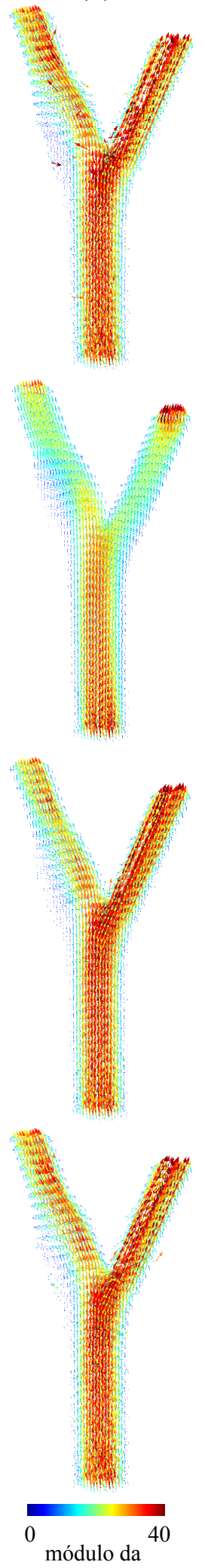

velocidade $(\mathrm{cm} / \mathrm{s})$ (d)
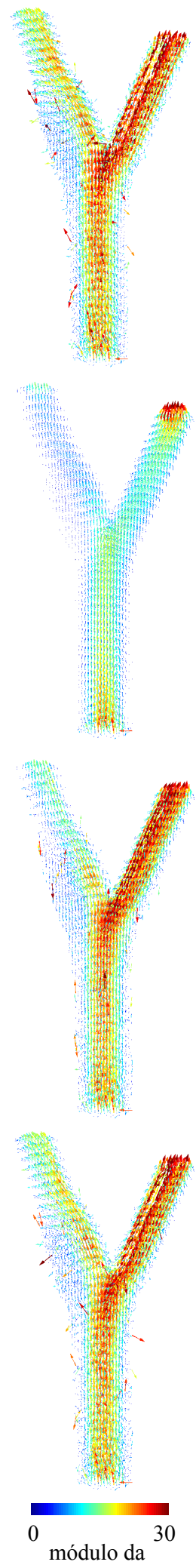

velocidade $(\mathrm{cm} / \mathrm{s})$

Figura 4.8: Visualização do campo de velocidades do fluido do phantom para os diferentes tipos de aquisição, em quatro instantes de tempo diferentes: (a) fase 2; (b) fase 3; (c) fase 4; (d) fase 5. $\mathrm{Na}$ figura, cada linha representa um dos métodos de estimação: contraste de fase (CF), simulação numérica pura (DFC), simulação numérica guiada pela componente de velocidade do contraste de fase na direção $z$ (DFC $+1 \mathrm{D}$ CF) e solução numérica guiada pelas três componentes de velocidade do contraste de fase $(\mathrm{DFC}+3 \mathrm{D} \mathrm{CF})$. 


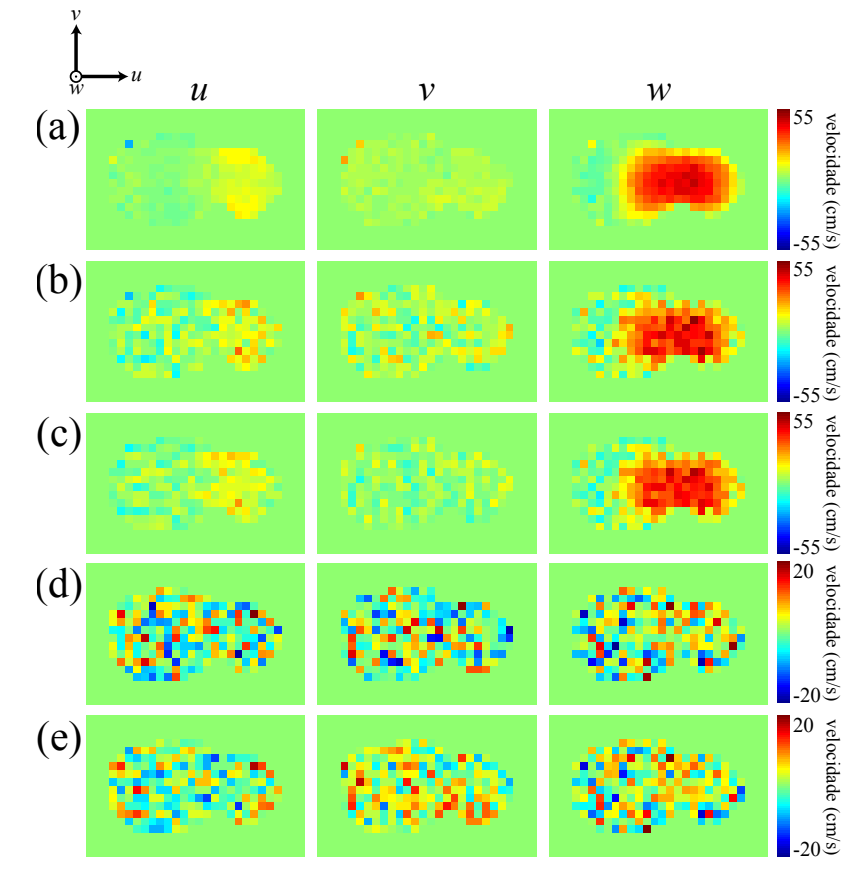

Figura 4.9: Componentes do campo de velocidade na bifurcação "carotídea" do phantom, na fase 4: (a) medido por CF; (b) medido por CF com ruído Gaussiano adicionado $(\sigma=7 \mathrm{~cm} / \mathrm{s})$; (c) simulação da DFC guiada pelas três direções de velocidade medida por CF com ruído Gaussiano adicionado; (d) diferença entre as imagens (b) e (a); e (e) diferença entre as imagens (c) e (a).

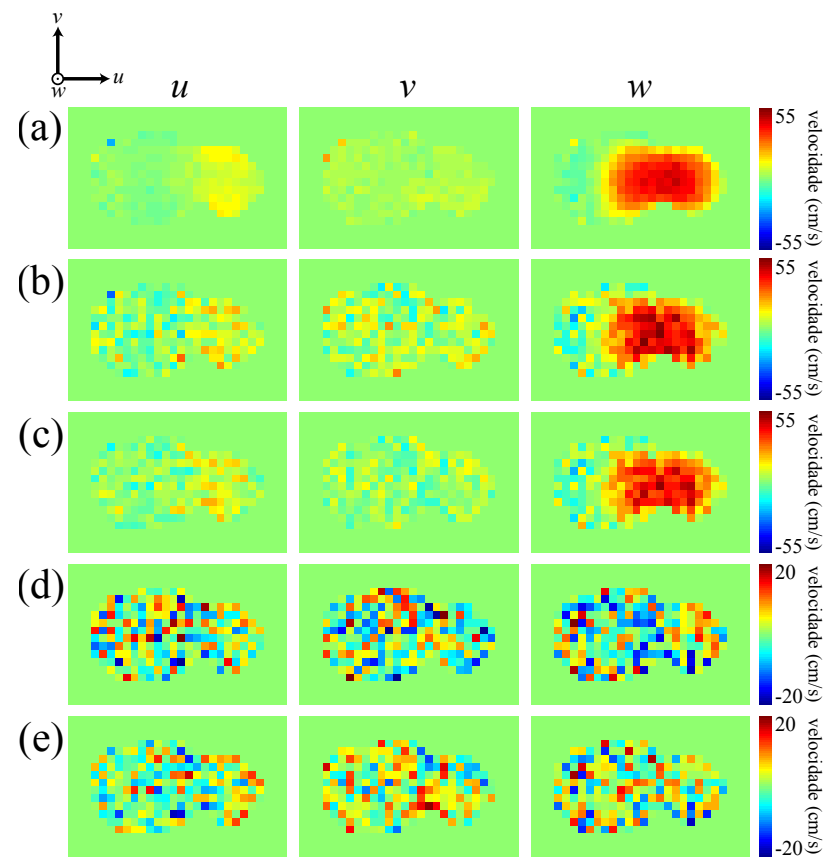

Figura 4.10: Componentes do campo de velocidade na bifurcação "carotídea" do phantom, na fase 4: (a) medido por CF; (b) medido por CF com ruído Gaussiano adicionado ( $\sigma=8 \mathrm{~cm} / \mathrm{s})$; (c) simulação da DFC guiada pelas três direções de velocidade medida por CF com ruído Gaussiano adicionado; (d) diferença entre as imagens (b) e (a); e (e) diferença entre as imagens (c) e (a). 


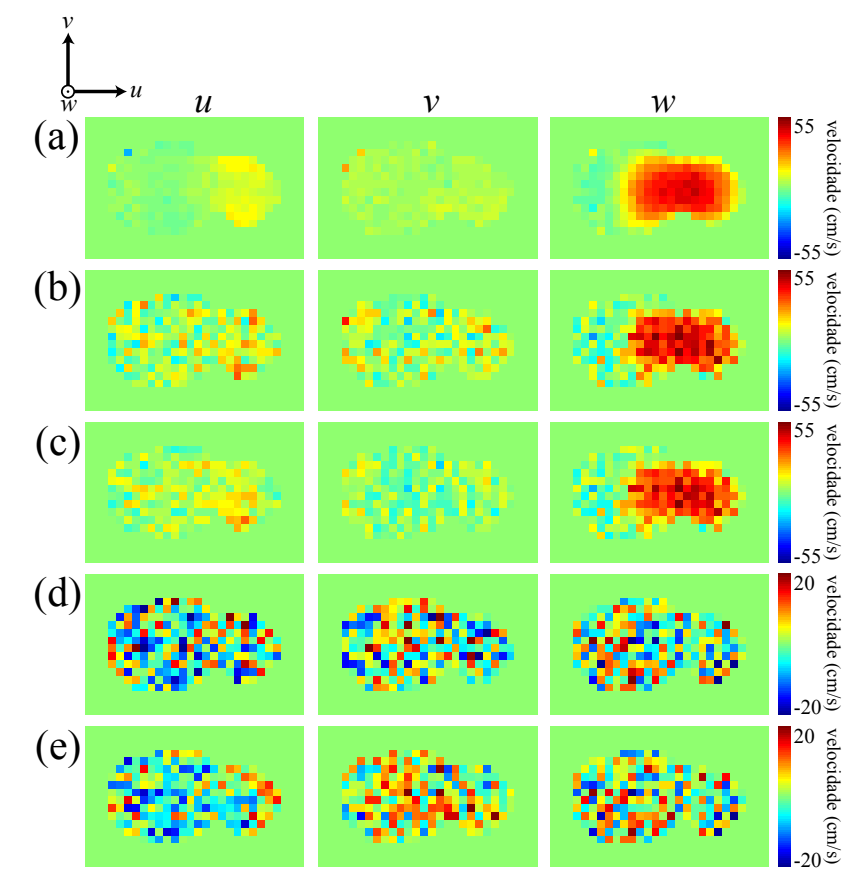

Figura 4.11: Componentes do campo de velocidade na bifurcação "carotídea" do phantom, na fase 4: (a) medido por CF; (b) medido por CF com ruído Gaussiano adicionado $(\sigma=9 \mathrm{~cm} / \mathrm{s})$; (c) simulação da DFC guiada pelas três direções de velocidade medida por CF com ruído Gaussiano adicionado; (d) diferença entre as imagens (b) e (a); e (e) diferença entre as imagens (c) e (a).

\begin{tabular}{cccc}
\hline Fase 2 & DFC & DFC + 1D CF & DFC + 3D CF \\
\hline $\mathrm{SER}_{u}$ & $2,15 \mathrm{~dB}$ & $2,00 \mathrm{~dB}(\downarrow)$ & $4,82 \mathrm{~dB}(\uparrow)$ \\
$\mathrm{SER}_{v}$ & $-0,12 \mathrm{~dB}$ & $-0,17 \mathrm{~dB}(\downarrow)$ & $2,36 \mathrm{~dB}(\uparrow)$ \\
$\mathrm{SER}_{w}$ & $6,98 \mathrm{~dB}$ & $16,56 \mathrm{~dB}(\uparrow \uparrow)$ & $12,79 \mathrm{~dB}(\uparrow)$ \\
\hline $\mathrm{SER}_{\vec{\nu}}$ & $4,62 \mathrm{~dB}$ & $6,39 \mathrm{~dB}(\uparrow)$ & $11,77 \mathrm{~dB}(\uparrow \uparrow)$ \\
\hline
\end{tabular}

Tabela 4.1: Relação sinal-erro entre o campo de velocidades medido com a ressonância magnética, $\vec{\nu}_{\mathrm{cf}}=\left(u_{\mathrm{cf}}, v_{\mathrm{cf}}, w_{\mathrm{cf}}\right)$, e os campos obtidos através das simulações numéricas guiadas, ou não, pelo contraste de fase, $\vec{\nu}_{\infty}=\left(u_{\infty}, v_{\infty}, w_{\infty}\right)$, para fase 2 .

\begin{tabular}{cccc}
\hline Fase 3 & DFC & DFC + 1D CF & DFC + 3D CF \\
\hline $\mathrm{SER}_{u}$ & $3,56 \mathrm{~dB}$ & $4,43 \mathrm{~dB}(\uparrow)$ & $6,64 \mathrm{~dB}(\uparrow)$ \\
$\mathrm{SER}_{v}$ & $-0,48 \mathrm{~dB}$ & $-0,54 \mathrm{~dB}(\downarrow)$ & $1,81 \mathrm{~dB}(\uparrow)$ \\
$\mathrm{SER}_{w}$ & $6,18 \mathrm{~dB}$ & $17,14 \mathrm{~dB}(\uparrow \uparrow)$ & $13,79 \mathrm{~dB}(\uparrow)$ \\
\hline $\mathrm{SER}_{\vec{\nu}}$ & $7,65 \mathrm{~dB}$ & $9,83 \mathrm{~dB}(\uparrow)$ & $14,71 \mathrm{~dB}(\uparrow \uparrow)$ \\
\hline
\end{tabular}

Tabela 4.2: Relação sinal-erro entre o campo de velocidades medido com a ressonância magnética, $\vec{\nu}_{\mathrm{cf}}=\left(u_{\mathrm{cf}}, v_{\mathrm{cf}}, w_{\mathrm{cf}}\right)$, e os campos obtidos através das simulações numéricas guiadas, ou não, pelo contraste de fase, $\vec{\nu}_{\infty}=\left(u_{\infty}, v_{\infty}, w_{\infty}\right)$, para fase 3 . 


\begin{tabular}{cccc}
\hline Fase 4 & DFC & DFC + 1D CF & DFC + 3D CF \\
\hline $\mathrm{SER}_{u}$ & $2,97 \mathrm{~dB}$ & $4,16 \mathrm{~dB}(\uparrow)$ & $6,74 \mathrm{~dB}(\uparrow)$ \\
$\mathrm{SER}_{v}$ & $-0,25 \mathrm{~dB}$ & $-0,30 \mathrm{~dB}(\downarrow)$ & $2,03 \mathrm{~dB}(\uparrow)$ \\
$\mathrm{SER}_{w}$ & $5,44 \mathrm{~dB}$ & $16,53 \mathrm{~dB}(\uparrow \uparrow)$ & $13,46 \mathrm{~dB}(\uparrow)$ \\
\hline $\mathrm{SER}_{\vec{\nu}}$ & $6,57 \mathrm{~dB}$ & $8,38 \mathrm{~dB}(\uparrow)$ & $13,13 \mathrm{~dB}(\uparrow \uparrow)$ \\
\hline
\end{tabular}

Tabela 4.3: Relação sinal-erro entre o campo de velocidades medido com a ressonância magnética, $\vec{\nu}_{\mathrm{cf}}=\left(u_{\mathrm{cf}}, v_{\mathrm{cf}}, w_{\mathrm{cf}}\right)$, e os campos obtidos através das simulações numéricas guiadas, ou não, pelo contraste de fase, $\vec{\nu}_{\infty}=\left(u_{\infty}, v_{\infty}, w_{\infty}\right)$, para fase 4 .

\begin{tabular}{cccc}
\hline Fase 5 & DFC & DFC + 1D CF & DFC + 3D CF \\
\hline $\mathrm{SER}_{u}$ & $1,85 \mathrm{~dB}$ & $2,66 \mathrm{~dB}(\uparrow)$ & $5,65 \mathrm{~dB}(\uparrow)$ \\
$\mathrm{SER}_{v}$ & $-0,08 \mathrm{~dB}$ & $-0,20 \mathrm{~dB}(\downarrow)$ & $2,10 \mathrm{~dB}(\uparrow)$ \\
$\mathrm{SER}_{w}$ & $3,59 \mathrm{~dB}$ & $14,01 \mathrm{~dB}(\uparrow \uparrow)$ & $11,06 \mathrm{~dB}(\uparrow)$ \\
\hline $\mathrm{SER}_{\vec{\nu}}$ & $2,79 \mathrm{~dB}$ & $4,11 \mathrm{~dB}(\uparrow)$ & $10,17 \mathrm{~dB}(\uparrow \uparrow)$ \\
\hline
\end{tabular}

Tabela 4.4: Relação sinal-erro entre o campo de velocidades medido com a ressonância magnética, $\vec{\nu}_{\mathrm{cf}}=\left(u_{\mathrm{cf}}, v_{\mathrm{cf}}, w_{\mathrm{cf}}\right)$, e os campos obtidos através das simulações numéricas guiadas, ou não, pelo contraste de fase, $\vec{\nu}_{\infty}=\left(u_{\infty}, v_{\infty}, w_{\infty}\right)$, para fase 5 .

\begin{tabular}{l|rc|rc|rr}
\hline & \multicolumn{2}{|c|}{$\boldsymbol{\sigma}=\mathbf{7} \mathbf{~ c m} / \mathbf{s}$} & \multicolumn{2}{c|}{$\boldsymbol{\sigma}=\mathbf{8} \mathbf{~ c m} / \mathbf{s}$} & \multicolumn{2}{c}{$\boldsymbol{\sigma}=\mathbf{9} \mathbf{~ c m} / \mathbf{s}$} \\
\hline & \multicolumn{1}{c}{$\mathbf{C F}$} & & $\mathbf{C F}$ & & \multicolumn{1}{c}{$\mathbf{C F}$} \\
& Ruidoso & DFC+3D & Ruidoso & DFC $+3 \mathbf{3 D}$ & Ruidoso & DFC+3D \\
\hline $\mathrm{SER}_{u}$ & $3,90 \mathrm{~dB}$ & $4,47 \mathrm{~dB}(\uparrow)$ & $3,41 \mathrm{~dB}$ & $4,16 \mathrm{~dB}(\uparrow)$ & $2,87 \mathrm{~dB}$ & $3,72 \mathrm{~dB}(\uparrow)$ \\
$\mathrm{SER}_{v}$ & $-0,24 \mathrm{~dB}$ & $-0,01 \mathrm{~dB}(\uparrow)$ & $-0,79 \mathrm{~dB}$ & $-0,28 \mathrm{~dB}(\uparrow)$ & $-1,33 \mathrm{~dB}$ & $-0,63 \mathrm{~dB}(\uparrow)$ \\
$\mathrm{SER}_{w}$ & $8,11 \mathrm{~dB}$ & $8,53 \mathrm{~dB}(\uparrow)$ & $7,51 \mathrm{~dB}$ & $7,88 \mathrm{~dB}(\uparrow)$ & $5,58 \mathrm{~dB}$ & $7,09 \mathrm{~dB}(\uparrow)$ \\
\hline $\mathrm{SER}_{\vec{\nu}}$ & $7,81 \mathrm{~dB}$ & $8,81 \mathrm{~dB}(\uparrow)$ & $6,64 \mathrm{~dB}$ & $7,87 \mathrm{~dB}(\uparrow)$ & $5,93 \mathrm{~dB}$ & $7,44 \mathrm{~dB}(\uparrow)$ \\
\hline
\end{tabular}

Tabela 4.5: Comparação da relação sinal-ruído entre as componentes de velocidade do phantom na fase 4 medidas por CF com ruído Gaussiano adicionado (para os diferentes valores de desvio padrão, $\sigma$ ) e as componentes de velocidade obtidas usando o CF ruidoso para guiar as simulações computacionais.

\subsection{Demonstração In Vivo}

\subsubsection{Aparato Experimental}

Os dados de contraste de fase in vivo utilizados neste capítulo foram cedidos pelo Pesquisador Jon-Friedrik Nielsen, do Laboratório de Ressonância Magnética Funcional da University of Michigan (Ann Arbor, Michigan, EUA), e são os mesmos dados utilizados na Ref. [48]. Esses foram obtidos através de imagens volumétricas TF3D fast gradient echo (FGRE) em quatro instantes distintos na artéria carótida de um indivíduo saudável (tamanho do voxel $1,0 \times 1,0 \times 2,5 \mathrm{~mm}^{3}$; campo de visão $7,5 \times 12,0 \times 16,0 \mathrm{~cm}^{3} ;$ TR 7,0 
ms; ângulo de flip de 15% resolução temporal $56 \mathrm{~ms}$; VENC $160 \mathrm{~cm} / \mathrm{s} ; 7$ minutos por medida), em um sistema GE Signa 3T EXCITE HD (40 mT/m de amplitude máxima do gradiente e $150 \mathrm{mT} / \mathrm{m} / \mathrm{s}$ de taxa máxima de variação) com um arranjo de bobinas de 4 canais para a carótida. O eixo perpendicular ao plano de corte $(z)$ foi orientado na direção S/I. O indivíduo assinou consentimento informado e foi examinado usando um protocolo aprovado pelos orgãos competentes da University of Southern California. As componentes de velocidade $u_{\mathrm{cf}}, v_{\mathrm{cf}}$ e $w_{\mathrm{cf}}$ foram calculadas usando os dados de apenas uma das bobinas. Assim como no caso do phantom, o lumén do vaso sanguíneo foi segmentado manualmente, limitando a fronteira do vaso em uma pilha de cortes axiais bidimensionais.

Os campos magnéticos variáveis com o tempo produzidos pelos gradientes nas sequências de pulso induzem correntes elétricas nas componentes condutoras de corrente dentro do equipamento de RM, como as próprias bobinas de gradiente e também as bobinas de RF [76]. Essas correntes induzidas são chamadas de eddy currents e criam campos magnéticos indesejados que são capazes de gerar artefatos na imagem. Nas máquinas modernas de RM os efeitos de eddy currents são mínimos. No entanto, já foram relatados erros quantitativos nas medidas de velocidade utilizando o contraste de fase devido a esse efeito [77]. No constraste de fase, o efeito indesejado aparece na forma de desníveis residuais lineares da velocidade. Esses são facilmente removidos através de uma interpolação linear definida dentro da região tridimensional contendo apenas tecido estático. Assim, para as medidas de constraste de fase in vivo, esses efeitos foram percebidos e removidos para todas as componentes de velocidade. Vale observar que esses efeitos não foram detectados para os dados obtidos com o phantom.

\subsubsection{Experimentos}

Com os dados adquiridos in vivo, está sendo realizado um experimento igual àquele realizado para o phantom, sendo simulados três campos de velocidade: a solução numérica pura (que utilizava os dados do contraste de fase apenas como condições de contorno do vaso sanguíneo), a solução numérica influenciada pela medida de velocidade $w_{\text {cf }}$ (direção principal do escoamento) e, finalmente, a solução numérica que incorpora todas as três direções de velocidade $\left(u_{\mathrm{cf}}, v_{\mathrm{cf}}\right.$ e $\left.w_{\mathrm{cf}}\right)$. Novamente, os três campos de velocidade foram comparados quantitativamente - utilizando a relação sinal-erro - e qualitativamente com as medidas obtidas por contraste de fase. 
Novamente, para cada instante de tempo computacional $n$, foi necessária apenas uma iteração para a convergência da equação da pressão (Eq. (3.32), com tempo de processamento de aproximadamente de 3 minutos por iteração. Para a simulação numérica pura, foram necessárias em média 390 iterações até a convergência; a simulação guiada com a velocidade $w_{\text {cf }}$ precisou em média de 265 iterações até a convergência; e, finalmente, a simulação guiada com as três componentes de velocidade necessitou em média de apenas 7 iterações; considerando $\varepsilon=10^{-3}$ na Eq. (4.4). Faz-se necessário observar que as simulações do fluxo in vivo requereram mais iterações para convergir porque foram realizadas em uma malha mais refinada que as simulações do escoamento do phantom. Além disso, é importante ressaltar que, nas simulações realizadas por Nielsen \& Nayak [48], os quais utilizaram esse mesmo conjunto de dados, eram necessárias aproximadamente 5 iterações para convergência da equação da pressão, sendo que cada iteração demorava 3 minutos em média. Isso mostra que o algoritmo implementado neste trabalho é mais eficiente que a implementação desenvolvida por Nielsen \& Nayak.

Tendo em vista que os dados in vivo possuem resolução espacial relativamente baixa, esses foram reconstruídos em uma grade de $0,5 \times 0,5 \times 1,25 \mathrm{~mm}^{3}$. Assim, os dados foram interpolados com um fator de 2 vezes em cada um dos eixos $(x, y$ e $z$ ). Neste caso, as matrizes $\mathbf{A}_{u, \mathrm{cf}}, \mathbf{A}_{v, \mathrm{cf}}$, e $\mathbf{A}_{w, \mathrm{cf}}$, definidas nas Eqs. (4.8) a (4.10), são matrizes simétricas $L \times L$ com múltiplas diagonais, em que as entradas da matriz são dadas pela função de espalhamento de ponto $\psi(x, y, z)=\operatorname{sinc}(x / \delta x) \cdot \operatorname{sinc}(y / \delta y) \cdot \operatorname{sinc}(z / \delta z), \operatorname{com} \delta x=1 \mathrm{~mm}$, $\delta y=1 \mathrm{~mm}$ e $\delta z=2,5 \mathrm{~mm}$.

Para os cálculos computacionais, iremos supor que as constantes de viscosidade e densidade como sendo $\mu=0,0032 \mathrm{~Pa} \cdot \mathrm{s}$ e $\rho=1060 \mathrm{~kg} / \mathrm{m}^{3}$, respectivamente [78]. Para os dados in vivo, os cálculos do algoritmo foram feitos em uma malha Cartesiana com uma resolução $0,5 \times 0,5 \times 1,25 \mathrm{~mm}^{3}$ e um passo de tempo computacional $\delta t=0,25 \mathrm{~ms}$. O domínio de cálculo da solução numérica era retangular de tamanho $30 \times 37 \times 125 \mathrm{~mm}^{3}$. O procedimento para construção de soluções influenciadas tanto pela dinâmica dos fluidos computacional quanto pelos dados medidos por ressonância magnética foi realizado de forma independente para cada um dos quatro instantes de tempo medidos.

Para os conjuntos de dados obtidos in vivo, também foi calculada a relação sinalerro com o objetivo de quantificar as diferenças entre os conjuntos. Novamente, a SER foi calculada individualmente para cada uma das componentes de velocidade das simulações 
realizadas, nos diferentes instantes de tempo do ciclo cardíaco, e também para o vetor velocidade, $\vec{\nu}$.

\subsubsection{Resultados}

Nas Figuras 4.12, 4.13 e 4.14 são mostradas as componentes de velocidade $u, v$ e $w$, para três cortes transversais da artéria carótida do indivíduo, em quatro instantes de tempo diferentes do ciclo cardíaco. Nessas figuras, é feita uma comparação entre as velocidades medidas através de ressonância magnética por contraste de fase e aquelas obtidas por meio de simulações de DFC produzidas sem influência do contraste de fase; com a influência somente da velocidade $w_{\text {cf }}$; e guiada pelas medições nas três direções.

É apresentado na Fig. 4.15 o divergente do campo de velocidades para a medida por contraste de fase e também das soluções numéricas. Assim como nos resultados do phantom, observamos que o campo de velocidades medido por contraste de fase não satisfaz a equação da continuidade (Eq. (3.8)). Nesse caso, além do ruído e dos efeitos de falta de homogeneidade do campo magnético $B_{0}$ e volume parcial, a elasticidade dos vasos sanguíneos também é um fator que contribui para que o campo de velocidades não satisfaça a equação da continuidade. Este efeito não se faz presente na análise anterior (seção 4.2), pois os tubos do phantom utilizado naquele experimento são todos rígidos.

Assim como os resultados observados para o phantom, a utilização dos campos de velocidade medidos para guiar as soluções computacionais leva a soluções que se assemelham com as medidas do contraste de fase mas que, ao mesmo tempo, satisfazem as equações da mecânica dos fluidos (Figs. 4.12 a 4.15). Novamente, a solução numérica pura - cuja única influência das medidas reais está nas condições de fronteira de entrada e saída — produz soluções que satisfazem o modelo físico, mas, que, qualitativamente e quantitativamente, são consideravelmente diferentes das medidas reais. Também é possível perceber, em uma comparação entre as Figs. 4.12, 4.13 e 4.14, que a utilização da componente de velocidade na direção principal do escoamento (eixo z) produz uma solução que satisfaz o modelo físico, mas, tendo em vista que $u$ e $v$ são obtidos pela a simulação numérica sem informação adicional das medidas de RM, elas são qualitativamente diferentes das componentes medidas com o contraste de fase. Já com a DFC guiada por RM nas três direções, vê-se claramente que os resultados são os que mais se assemelham às medidas de constraste de fase in vivo. 
As Tabelas 4.6 a 4.9 mostram os valores da SER calculados para cada abordagem, em cada fase cardíaca. Nota-se que o erro de estimação é mais severo nas componentes de velocidade no plano $x-y$, como já observado qualitativamente (Figs. 4.12 a 4.15). Isso é natural, pois sendo o movimento do sangue predominantemente na direção do eixo $z$, o sinal da componente $w_{\mathrm{cf}}$ é maior. Logo, essa dimensão possui maior SER. Para a fase 3, por exemplo, o sinal da componente $w_{\text {cf }}$ possui $29,76 \mathrm{~dB}$ a mais de energia que a componente $u_{\text {cf }}$ e $27,09 \mathrm{~dB}$ a mais que a componente $v_{\text {cf }}$. No entanto, diferentemente dos resultados obtidos para o phantom, os valores de SER das componentes no plano $x-y$ para a solução numérica guiada apenas pela componente de velocidade $w_{\mathrm{cf}}$, em alguns casos, são consideravelmente menores que as componentes de velocidade, no mesmo plano, da solução numérica pura. Isso é justificado pelo fato de que foi usado o mesmo VENC para a aquisição das três componentes de velocidade no contraste de fase. Porém, o valor do VENC usado é significativemente maior que os valores das componentes de velocidade no plano $x-y$. Dessa forma, a relação sinal-ruído para essas componentes é agravada drasticamente com relação à componente de velocidade na direção z. Além disso, verifica-se uma perda maior de SER com relação ao contraste de fase comparado à simulação numérica pura. Finalmente, percebe-se mais uma vez que, em geral, a simulação que tem a melhor SER para o campo de velocidades $\vec{\nu}$, ou seja, que mais se aproxima do contraste de fase, é aquela que se utiliza das três componentes de velocidade como guia.

Finalmente, na Fig. 4.16 são apresentados os campos de velocidade dos escoamentos do sanguíneos medidos por contraste de fase e também aqueles obtidos por meio de as simulações de DFC guiadas ou não por dados de ressonância magnética. Em uma comparação qualitativa entre a medida do contraste de fase e os campos simulados, percebe-se que as simulações guiadas pelo contraste de fase apresentam uma maior similaridade qualitativa com o escoamento medido do que a simulação computacional pura. Percebe-se que, na artéria carótida comum, o campo de velocidade medido e os campos de velocidade simulados apresentam comportamento similares. No entanto, após a bifurcação da carótida o campo de velocidades medido nota-se uma diferença significativa entre os campos de velocidade guiados pela RM e aqueles gerados apenas pela dinâmica dos fluidos computacional. Tais resultados aqui encontrados condizem com o que já havia sido observado nas Fig. 4.12 , 4.13 e 4.14 e também com os resultados quantitativos apresentados nas Tabelas 4.6 a 4.9. Além disso, o resultado para os dados in vivo são consistentes com aqueles apresentados no 
experimento com o phantom.

\subsection{Discussão e Conclusão}

Os métodos apresentados neste capítulo usam o algoritmo SIMPLER tridimensional, em malhas Cartesianas e uniformes, para gerar simulações numéricas do escoamento sanguíneo sobre a influência de perfis de velocidade tridimensionais, in vivo e de um phantom, adquiridos por contraste de fase. A escolha do algoritmo SIMPLER e da malha Cartesiana se justifica pela facilidade de implementação. Os resultados mostraram que o método combinado RM-DFC possui maior similaridade com as medidas por contraste de fase, que as simulações computacionais influenciadas pelas medidas de CF somente como condições de fronteira de entrada e saída nas artérias. Isso nos permite, considerando os dados da ressonância magnética como "ground truth", acreditar que a técnica combinada é clinicamente aceitável, pois esta permite corrigir os campos de velocidade medidos por CF, forçando-os a satisfazer as equações da mecânica dos fluidos (Eqs. (3.2) e (3.3)). Além disso, foi mostrado que essa é uma técnica capaz de reduzir o ruído nos campos de velocidade medidos através do contraste de fase.

Outro aspecto que sugere que o método que combina RM e DFC pode ser utilizada no ambiente clínico é a sua rápida convergência. Nos casos em que as componentes de velocidade em todas as direções espaciais foram usadas para influenciar as simulações numéricas, poucas iterações foram necessárias para fazer a redução de ruído e também para que as equações satisfizessem as equações da mecânica dos fluidos.

Além disso, esse método pode também ser usado como uma técnica para redução do tempo de aquisição. Algumas das possibilidades sugeridas para reduzir o tempo de exame utilizando o método proposto neste capítulo são: (i) adquirir os dados de RM com resolução espacial reduzida e usar o método proposto para obter campos de velocidade com melhor resolução espacial; (ii) adquirir porções do volume (por exemplo, a entrada e a saída da carótida) com resolução espacial fina, enquanto que nas demais partes do volume os dados são adquiridos com resolução espacial menor; (iii) adquirir apenas alguns poucos cortes em volta da bifurcação e utilizar as simulações numéricas para preencher os espaços; (iv) medir uma ou duas componentes de velocidade com resolução espacial fina, enquanto que a(s) outra(s) é/são medida(s) com uma resolução espacial mais grosseira; (v) medir 
uma ou duas componentes de velocidade em todo o volume, enquanto que a(s) outra(s) componente(s) é(são) medida(s) somente em alguns cortes; e (vi) qualquer combinação de algumas dessas abordagens.

Nielsen \& Nayak [48] utilizaram apenas uma dimensão de velocidade para guiar as simulações computacionais e fizeram apenas uma comparação qualitativa em seu trabalho. Os resultados apresentados aqui verificam que tal método é muito eficaz para corrigir campos de velocidade medidos com ressonância magnética. No entanto, a utilização de apenas uma dimensão de velocidade para guiar a simulação numérica pode levar a soluções que satisfazem o modelo físico, mas diferem qualitativavemente e quantitativamente do campo de velocidades medido pela ressonância. Desta forma, como foi apresentado nos resultados, acreditamos que a utilização das três direções de velocidade medidas para guiar a solução computacional seja a melhor forma (qualitativamente e quantitativamente) de corrigir o escoamento medido com a ressonância magnética através de um modelo físico. Além disso, esta acarreta em um menor custo computacional. 


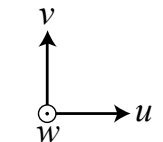

(a)

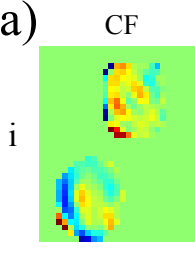

i

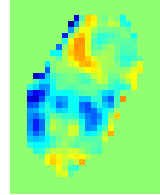

iii

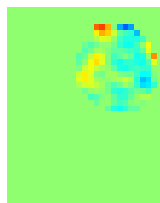

(c)
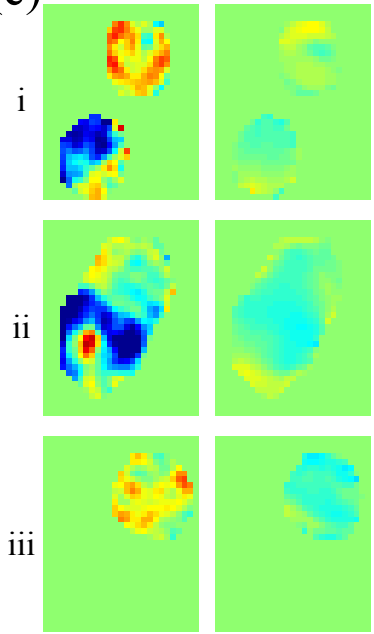
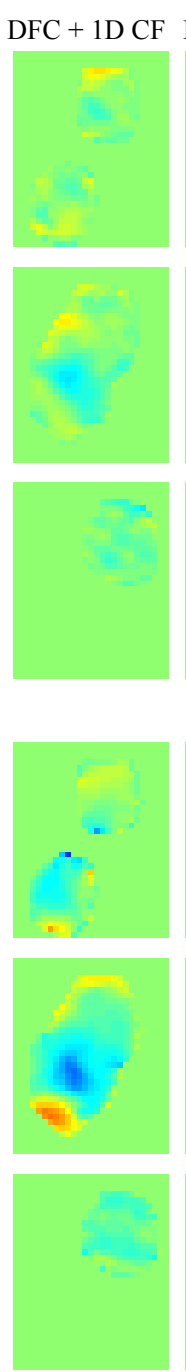

(b)
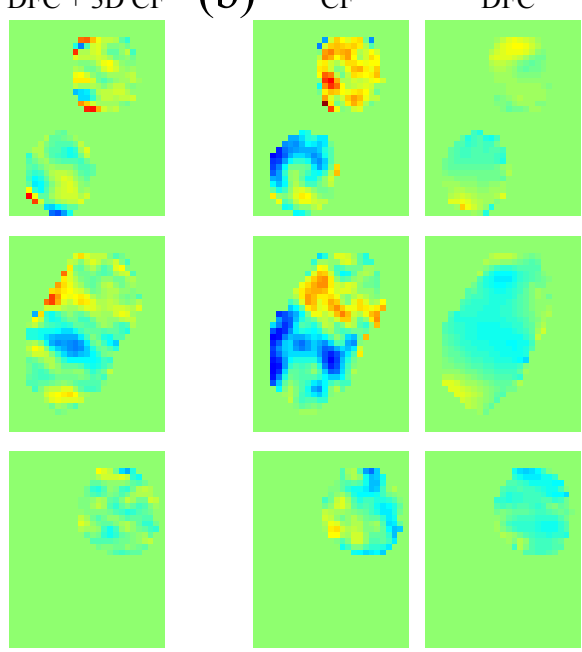

(d)
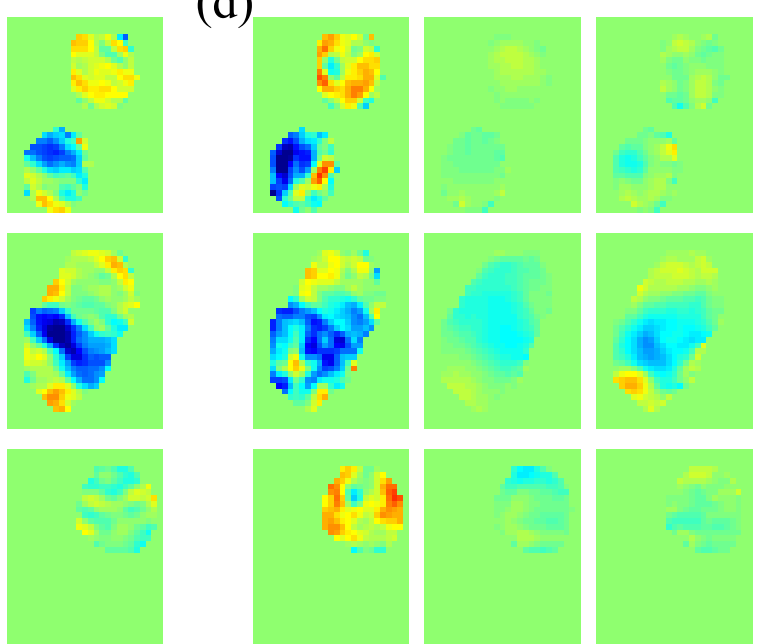


Figura 4.12: Seções transversais da componente de velocidade na direção $u$, em quatro instantes do ciclo cardíaco: (a) fase 1; (b) fase 2; (c) fase 3; (d) fase 4. Na figura, são apresentados três diferentes cortes da artéria carótida, a saber: (iii) carótida comum, (ii) bifurcação da carótida e (i) carótida bifurcada. As colunas representam os diferentes métodos de estimação: contraste de fase $(\mathrm{CF})$, simulação numérica pura (DFC), simulação numérica guiada pela componente de velocidade do contraste de fase na direção $z(\mathrm{DFC}+1 \mathrm{D} \mathrm{CF})$ e solução numérica guiada pelas três componentes de velocidade do contraste de fase (DFC $+3 \mathrm{D} \mathrm{CF})$. 


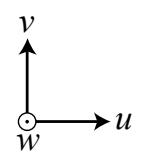

(a)
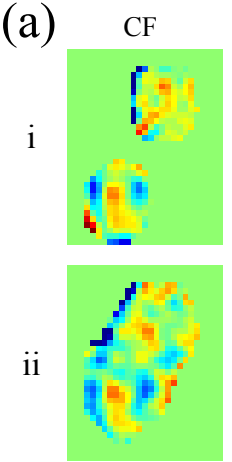

iii

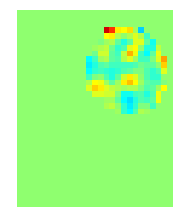

(c)
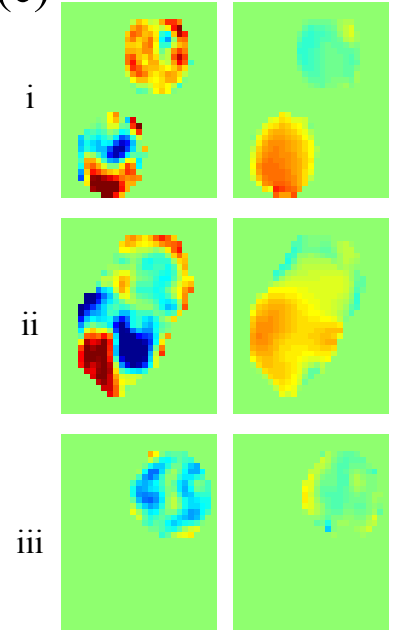

DFC
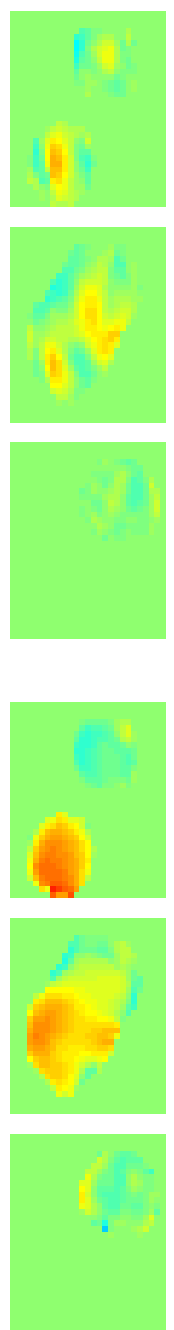
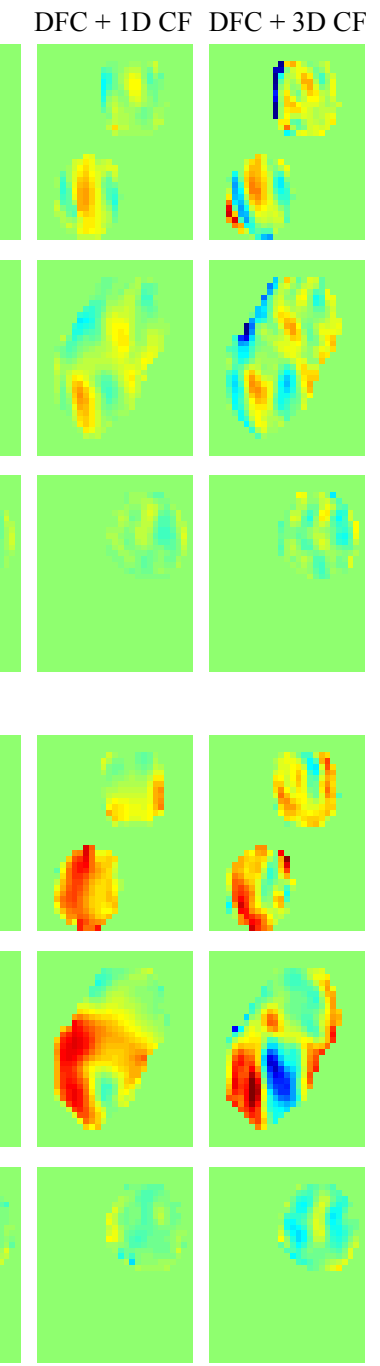

(b)
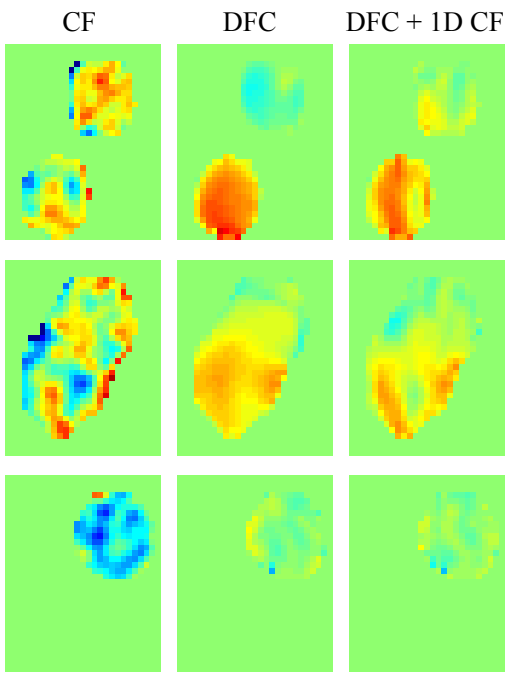

(d)
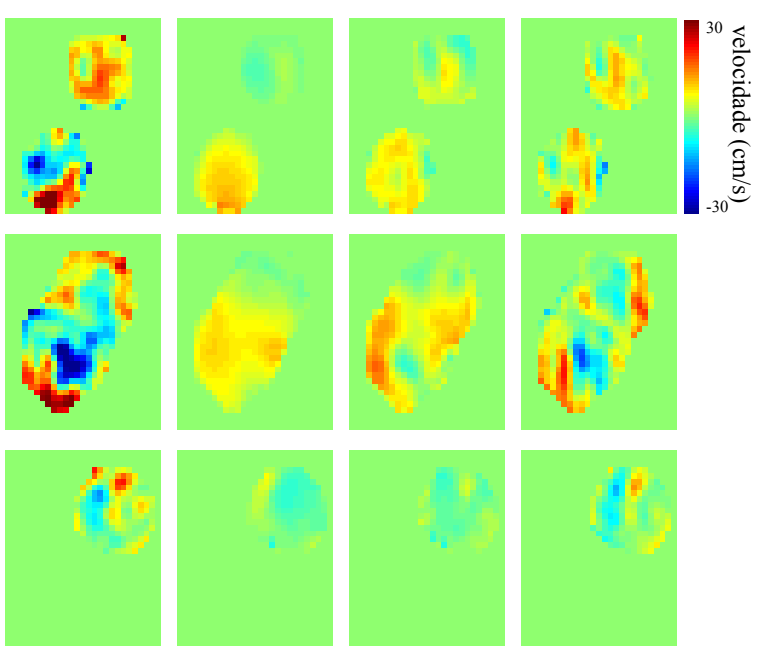
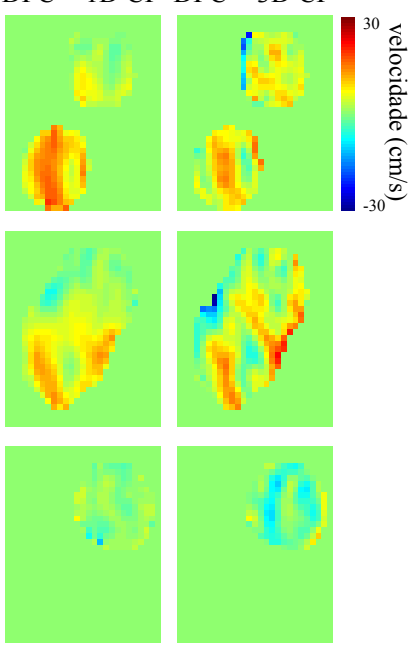

Figura 4.13: Seções transversais da componente de velocidade na direção $v$, em quatro instantes do ciclo cardíaco: (a) fase 1; (b) fase 2; (c) fase 3; (d) fase 4 . Na figura, são apresentados três diferentes cortes da artéria carótida, a saber: (iii) carótida comum, (ii) bifurcação da carótida e (i) carótida bifurcada. As colunas representam os diferentes métodos de estimação: contraste de fase $(\mathrm{CF})$, simulação numérica pura $(\mathrm{DFC})$, simulação numérica guiada pela componente de velocidade do contraste de fase na direção $z(\mathrm{DFC}+1 \mathrm{D} \mathrm{CF})$ e solução numérica guiada pelas três componentes de velocidade do contraste de fase $(\mathrm{DFC}+3 \mathrm{D} \mathrm{CF})$. 




(a)
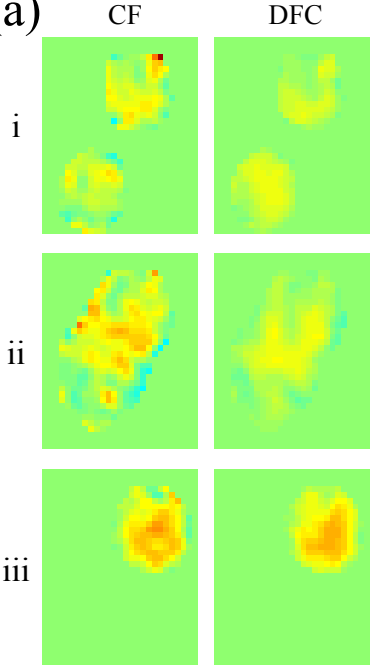

(c)
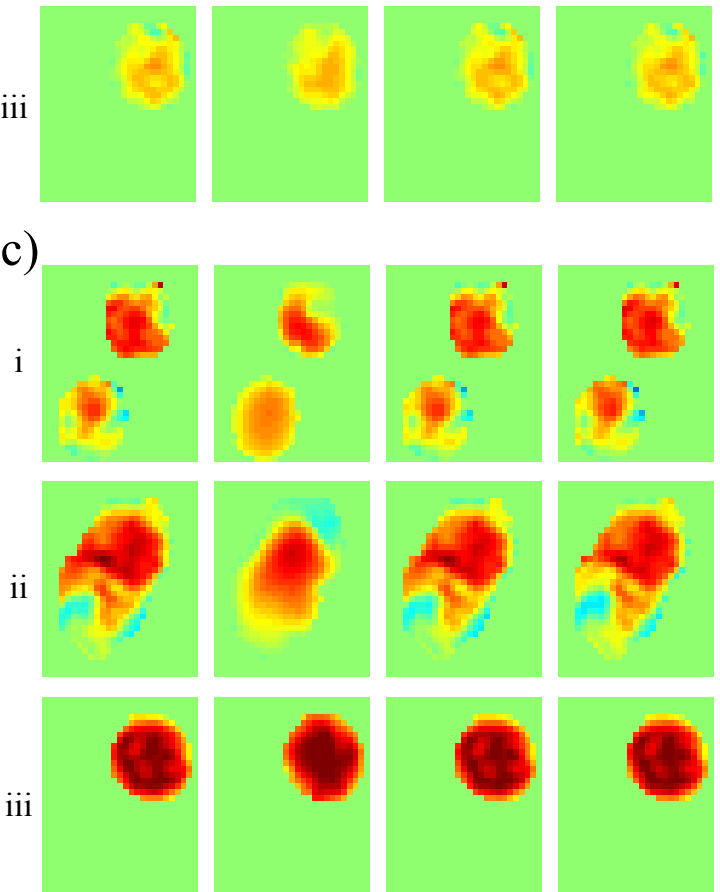

(b)
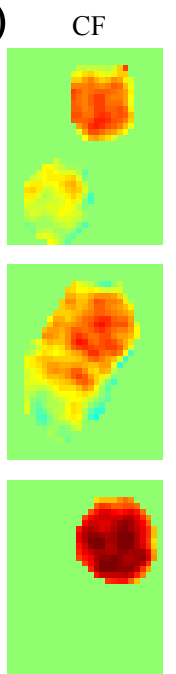

(d)
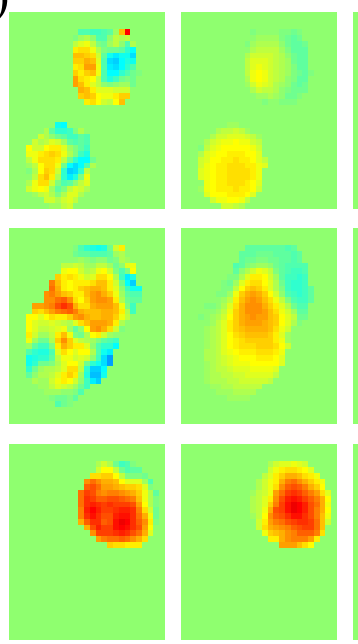
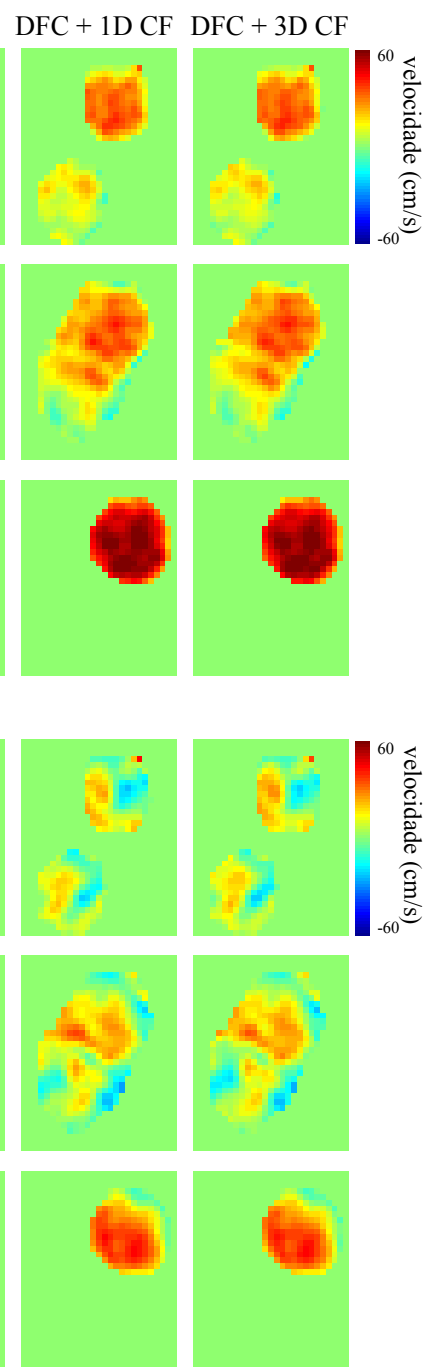

Figura 4.14: Seções transversais da componente de velocidade na direção $w$, em quatro instantes do ciclo cardíaco: (a) fase 1; (b) fase 2; (c) fase 3; (d) fase 4 . Na figura, são apresentados três diferentes cortes da artéria carótida, a saber: (iii) carótida comum, (ii) bifurcação da carótida e (i) carótida bifurcada. As colunas representam os diferentes métodos de estimação: contraste de fase $(\mathrm{CF})$, simulação numérica pura (DFC), simulação numérica guiada pela componente de velocidade do contraste de fase na direção $z(\mathrm{DFC}+1 \mathrm{D} \mathrm{CF})$ e solução numérica guiada pelas três componentes de velocidade do contraste de fase (DFC $+3 \mathrm{D} \mathrm{CF})$. 


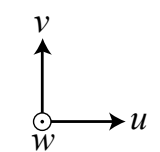

(a) $\quad \mathrm{CF}$

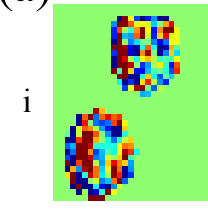

i

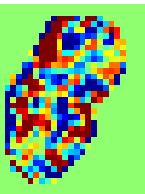

iii

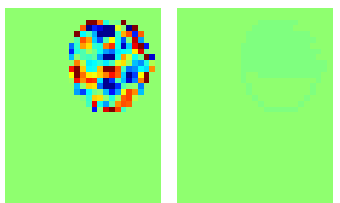

(c)
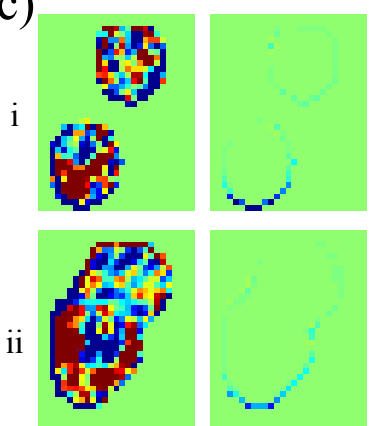

iii

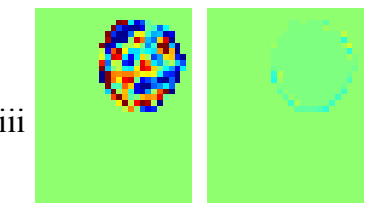

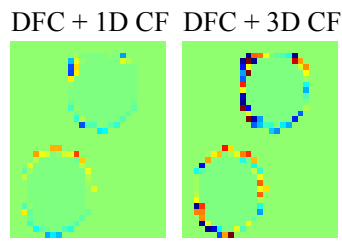
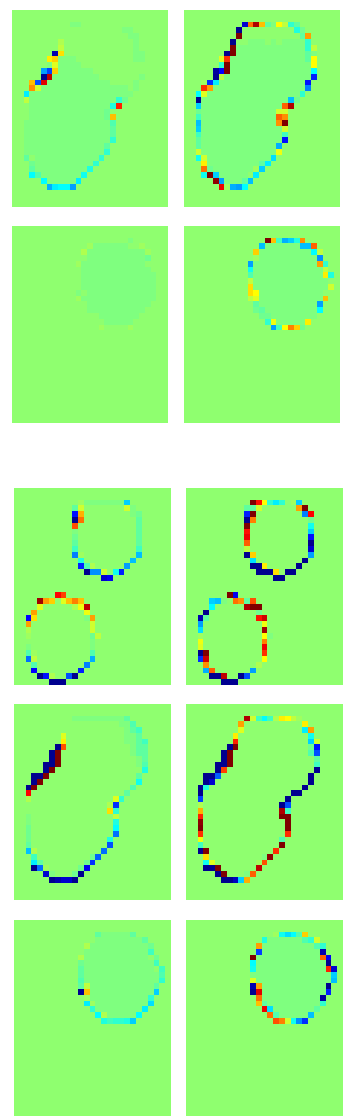

(b)
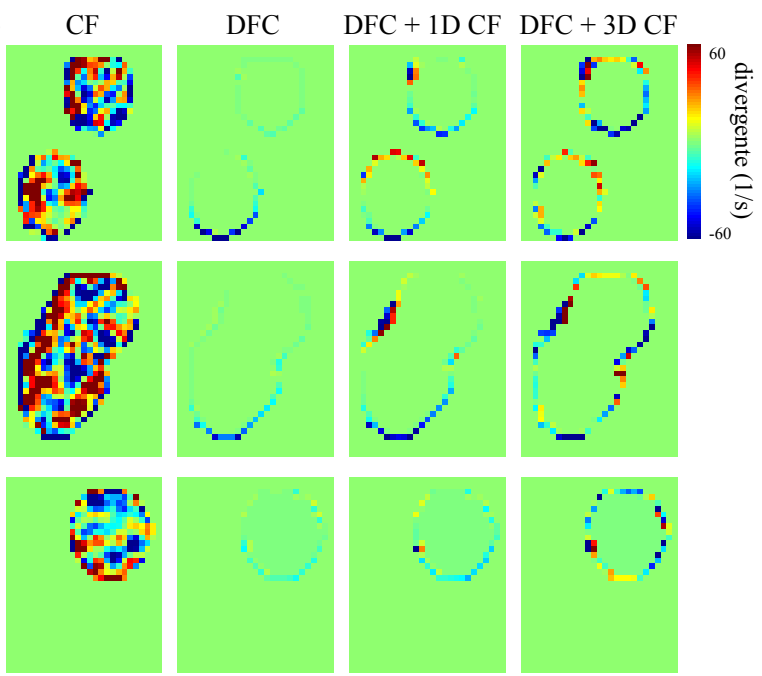

(d)
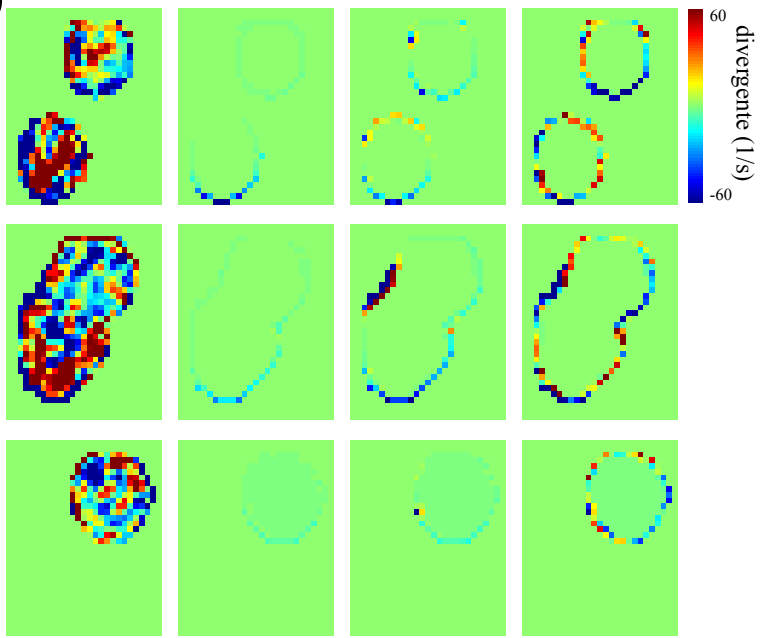

Figura 4.15: Seções transversais do $\operatorname{div}(\vec{\nu})$ em quatro instantes do ciclo cardíaco: (a) fase 1; (b) fase 2; (c) fase 3; (d) fase 4. Na figura, são apresentados três diferentes cortes da artéria carótida, a saber: (iii) carótida comum, (ii) bifurcação da carótida e (i) carótida bifurcada. As colunas representam os diferentes métodos de estimação: contraste de fase $(\mathrm{CF})$, simulação numérica pura (DFC), simulação numérica guiada pela componente de velocidade do contraste de fase na direção $z(\mathrm{DFC}+1 \mathrm{D} \mathrm{CF})$ e solução numérica guiada pelas três componentes de velocidade do contraste de fase $(\mathrm{DFC}+3 \mathrm{D} \mathrm{CF})$. 
(a)
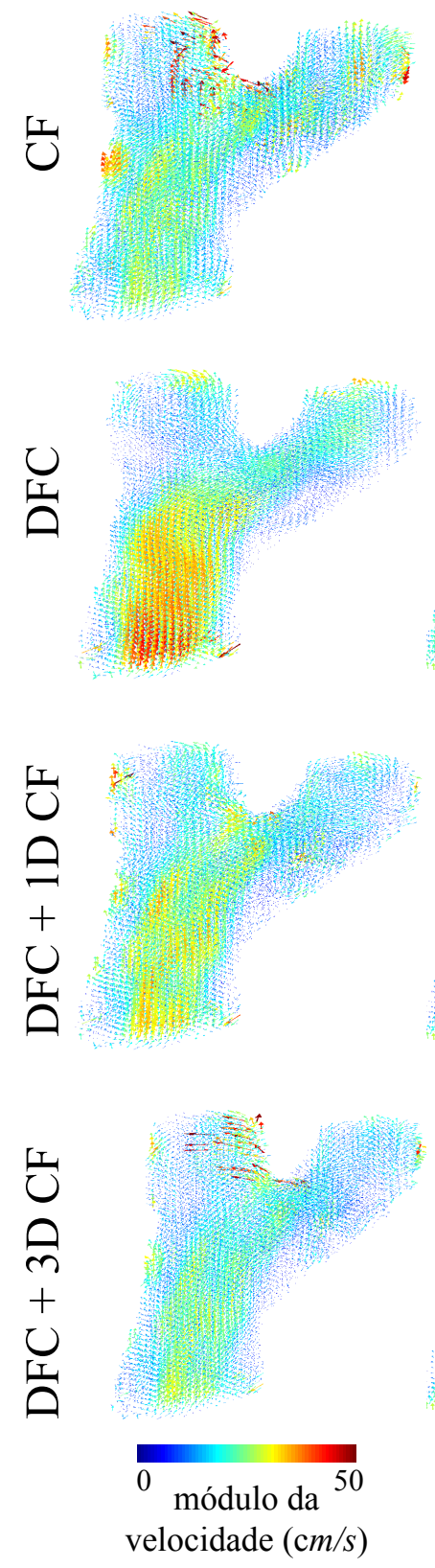

(b)
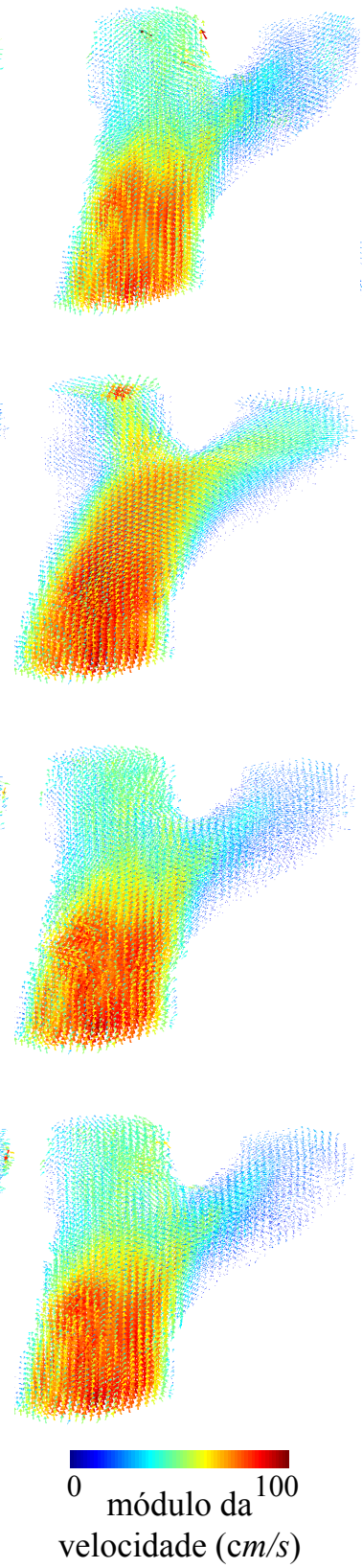

(c)
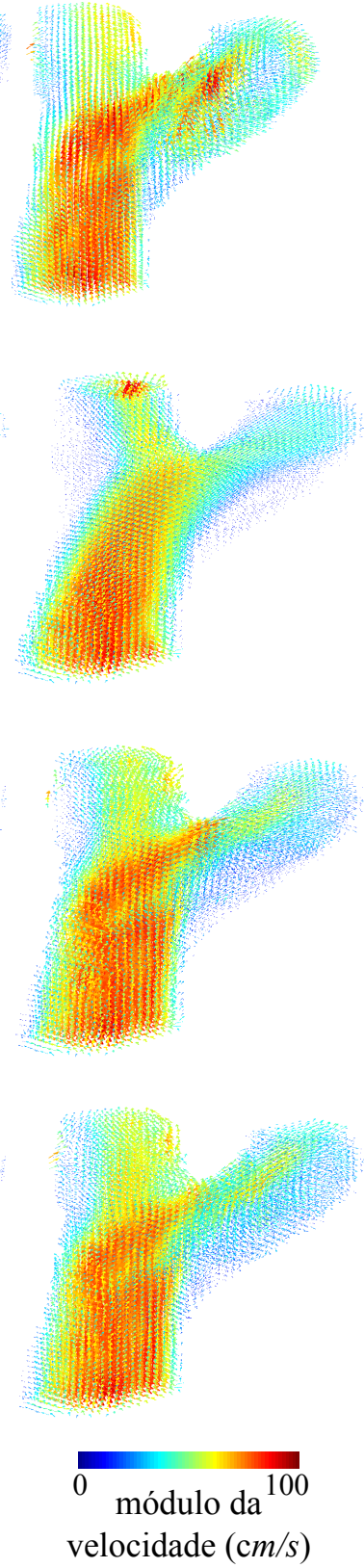

(d)
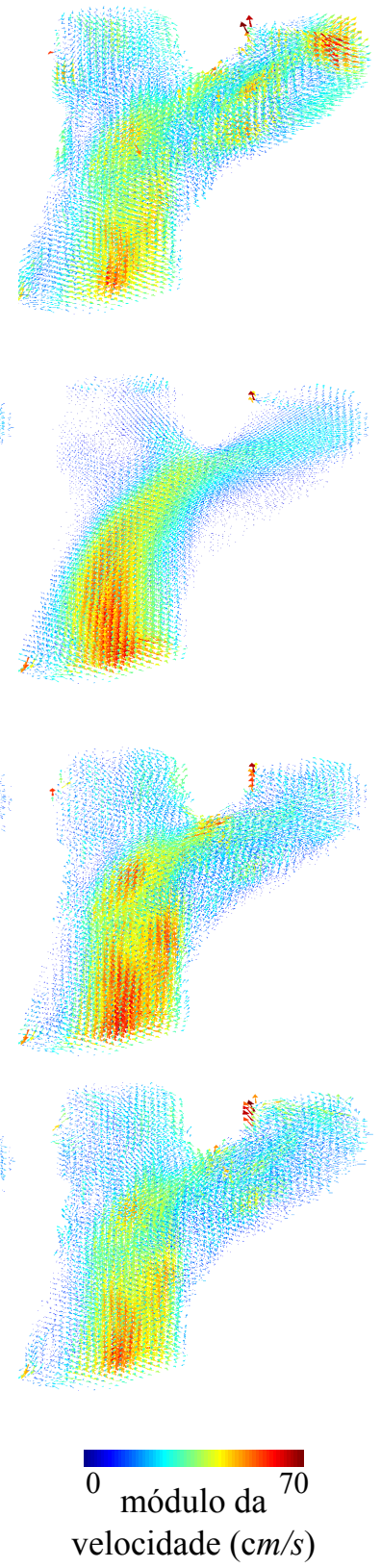

Figura 4.16: Visualização do escoamento do sangue na artéria carótida para os diferentes tipos de estimação em quatro instantes do ciclo cardíaco: (a) fase 1; (b) fase 2 ; (c) fase 3; (d) fase $4 . \mathrm{Na}$ figura, as linhas representam os diferentes métodos de estimação: contraste de fase (CF), simulação numérica pura (DFC), simulação numérica guiada pela componente de velocidade do contraste de fase na direção $z$ (DFC $+1 \mathrm{D} C F)$ e solução numérica guiada pelas três componentes de velocidade do contraste de fase $(\mathrm{DFC}+3 \mathrm{D} \mathrm{CF})$. 


\begin{tabular}{cccc}
\hline Fase 1 & DFC & DFC + 1D CF & DFC + 3D CF \\
\hline $\mathrm{SER}_{u}$ & $-0,15 \mathrm{~dB}$ & $-0,17 \mathrm{~dB}(\approx)$ & $-0,19 \mathrm{~dB}(\approx)$ \\
$\mathrm{SER}_{v}$ & $-0,46 \mathrm{~dB}$ & $-0,48 \mathrm{~dB}(\approx)$ & $-0,01 \mathrm{~dB}(\uparrow)$ \\
$\mathrm{SER}_{w}$ & $4,50 \mathrm{~dB}$ & $13,22 \mathrm{~dB}(\uparrow \uparrow)$ & $10,60 \mathrm{~dB}(\uparrow)$ \\
\hline $\mathrm{SER}_{\vec{\nu}}$ & $0,16 \mathrm{~dB}$ & $0,25 \mathrm{~dB}(\uparrow)$ & $0,41 \mathrm{~dB}(\uparrow \uparrow)$ \\
\hline
\end{tabular}

Tabela 4.6: Relação sinal-erro entre o campo de velocidades medido in vivo com a ressonância magnética $\left(\vec{\nu}_{\mathrm{cf}}=\left(u_{\mathrm{cf}}, v_{\mathrm{cf}}, w_{\mathrm{cf}}\right)\right)$ e os campos obtidos através das simulações numéricas guiadas, ou não, pelo contraste de fase, $\vec{\nu}_{\infty}=\left(u_{\infty}, v_{\infty}, w_{\infty}\right)$, para fase 1 .

\begin{tabular}{cccc}
\hline Fase 2 & DFC & DFC + 1D CF & DFC + 3D CF \\
\hline $\mathrm{SER}_{u}$ & $0,71 \mathrm{~dB}$ & $0,56 \mathrm{~dB}(\downarrow)$ & $0,53 \mathrm{~dB}(\downarrow)$ \\
$\mathrm{SER}_{v}$ & $-2,34 \mathrm{~dB}$ & $-1,61 \mathrm{~dB}(\uparrow)$ & $-0,76 \mathrm{~dB}(\uparrow)$ \\
$\mathrm{SER}_{w}$ & $4,61 \mathrm{~dB}$ & $16,98 \mathrm{~dB}(\uparrow \uparrow)$ & $15,31 \mathrm{~dB}(\uparrow)$ \\
\hline $\mathrm{SER}_{\vec{\nu}}$ & $5,51 \mathrm{~dB}$ & $8,54 \mathrm{~dB}(\uparrow)$ & $8,91 \mathrm{~dB}(\uparrow \uparrow)$ \\
\hline
\end{tabular}

Tabela 4.7: Relação sinal-erro entre o campo de velocidades medido in vivo com a ressonância magnética $\left(\vec{\nu}_{\mathrm{cf}}=\left(u_{\mathrm{cf}}, v_{\mathrm{cf}}, w_{\mathrm{cf}}\right)\right)$ e os campos obtidos através das simulações numéricas guiadas, ou não, pelo contraste de fase, $\vec{\nu}_{\infty}=\left(u_{\infty}, v_{\infty}, w_{\infty}\right)$, para fase 2 .

\begin{tabular}{cccc}
\hline Fase 3 & DFC & DFC + 1D CF & DFC + 3D CF \\
\hline $\mathrm{SER}_{u}$ & $0,51 \mathrm{~dB}$ & $0,50 \mathrm{~dB}(\approx)$ & $0,75 \mathrm{~dB}(\uparrow)$ \\
$\mathrm{SER}_{v}$ & $-1,51 \mathrm{~dB}$ & $-1,87 \mathrm{~dB}(\downarrow)$ & $-0,92 \mathrm{~dB}(\uparrow)$ \\
$\mathrm{SER}_{w}$ & $4,74 \mathrm{~dB}$ & $14,53 \mathrm{~dB}(\uparrow \uparrow)$ & $10,74 \mathrm{~dB}(\uparrow)$ \\
\hline $\mathrm{SER}_{\vec{\nu}}$ & $4,13 \mathrm{~dB}$ & $4,89 \mathrm{~dB}(\uparrow)$ & $6,28 \mathrm{~dB}(\uparrow \uparrow)$ \\
\hline
\end{tabular}

Tabela 4.8: Relação sinal-erro entre o campo de velocidades medido in vivo com a ressonância magnética $\left(\vec{\nu}_{\mathrm{cf}}=\left(u_{\mathrm{cf}}, v_{\mathrm{cf}}, w_{\mathrm{cf}}\right)\right)$ e os campos obtidos através das simulações numéricas guiadas, ou não, pelo contraste de fase, $\vec{\nu}_{\infty}=\left(u_{\infty}, v_{\infty}, w_{\infty}\right)$, para fase 3 .

\begin{tabular}{cccc}
\hline Fase 4 & DFC & DFC + 1D CF & DFC + 3D CF \\
\hline $\mathrm{SER}_{u}$ & $-0,02 \mathrm{~dB}$ & $0,32 \mathrm{~dB}(\uparrow)$ & $0,42 \mathrm{~dB}(\uparrow)$ \\
$\mathrm{SER}_{v}$ & $-0,86 \mathrm{~dB}$ & $-1,01 \mathrm{~dB}(\downarrow)$ & $-0,26 \mathrm{~dB}(\uparrow)$ \\
$\mathrm{SER}_{w}$ & $4,20 \mathrm{~dB}$ & $15,54 \mathrm{~dB}(\uparrow \uparrow)$ & $9,66 \mathrm{~dB}(\uparrow)$ \\
\hline $\mathrm{SER}_{\vec{\nu}}$ & $1,62 \mathrm{~dB}$ & $2,92 \mathrm{~dB}(\uparrow)$ & $4,03 \mathrm{~dB}(\uparrow \uparrow)$ \\
\hline
\end{tabular}

Tabela 4.9: Relação sinal-erro entre o campo de velocidades medido in vivo com a ressonância magnética $\left(\vec{\nu}_{\mathrm{cf}}=\left(u_{\mathrm{cf}}, v_{\mathrm{cf}}, w_{\mathrm{cf}}\right)\right)$ e os campos obtidos através das simulações numéricas guiadas, ou não, pelo contraste de fase, $\vec{\nu}_{\infty}=\left(u_{\infty}, v_{\infty}, w_{\infty}\right)$, para fase 4 . 


\section{Capítulo 5}

\section{Simulações Computacionais de}

\section{Escoamento Sanguíneo Guiadas por}

\section{Dados de RM com Codificação de}

\section{Velocidade em Fourier}

A codificação de velocidade em Fourier (CVF) é um método promissor para imageamento de fluxo por ressonância magnética na medição de escoamento sanguíneo cardiovascular. As distribuições adquiridas por CVF proporcionam maior relação sinal-ruído que o contraste de fase e também são robustas aos efeitos de volume parcial. No entanto, essas distribuições são usualmente adquiridas com baixa resolução espacial, devido ao longo tempo de aquisição relacionado a sua alta dimensionalidade $(x, y, w, t)$. Esses conjuntos de dados fornecem as distribuições de velocidade dentro de cada voxel, mas não fornecem diretamente mapas de velocidade similares aos do contraste de fase. Tais mapas de velocidade, entretanto, são úteis para calcular o verdadeiro escoamento dentro de um vaso sanguíneo ou, também, podem ser utilizados para guiar simulações computacionais, como mostrado no Capítulo 4.

Neste capítulo, é proposto um método capaz de extrair mapas de velocidade de alta resolução espacial a partir de distribuições de velocidade adquiridas com CVF de baixa resolução. A partir dos dados de contraste de fase adquiridos com o phantom, são simulados três conjuntos de dados com codificação de velocidade em Fourier com aquisição em espiral 
(eCVF) para diferentes valores de resolução espacial utilizando a componente de velocidade na direção principal do escoamento, $w_{\mathrm{cf}}$. Logo, são realizados experimentos para extrair das distribuições simuladas os mapas de velocidade, $\hat{w}_{\mathrm{cvf}}$, demonstrando a possibilidade de obter mapas de velocidade razoavelmente precisos a partir de distribuições adquiridas por CVF de baixa resolução espacial.

Devido a sua rapidez associada ao uso de trajetórias espirais na aquisição, é proposto neste capítulo, também, a utilização de dados de eCVF para guiar simulações de DFC. Com os mapas de velocidade extraídos das distribuições eCVF simuladas, são realizadas simulações de DFC guiadas com esses mapas de velocidade e as soluções encontradas são comparadas com a solução puramente computacional - i.e., com mínima influência da ressonância magnética - e também com a solução computacional guiada por uma componente de velocidade adquirida por contraste de fase $\left(w_{\mathrm{cf}}\right)$, considerando apenas 1 dos 9 NEX adquiridos. A solução guiada por apenas 1 NEX é utilizada para a comparação, pois o tempo de aquisição de uma componente de velocidade por contraste de fase, com os mesmos parâmetros utilizados neste trabalho, com resolução espacial $\delta r=0,5 \mathrm{~mm}$, é equivalente ao tempo de aquisição de uma distribuição eCVF com resolução espacial de $\delta r=1,0 \mathrm{~mm}$. Por fim, os resultados com o DFC guiado por eCVF foram comparados com os obtidos, no capítulo 4, utilizando para guiar a simulação de DFC somente a componente $w$ do contraste de fase $\left(w_{\mathrm{cf}}\right)$, mas utilizando dados com boa relação sinal-ruído (9 NEX).

A lista de publicações relacionadas aos resultados apresentados neste capítulo se encontra no Anexo I.

\subsection{Métodos}

Como mostrado no Capítulo 2, o modelo de sinal que incorpora os efeitos de truncamento do espaço $k$ de uma distribuição adquirida com CVF [25] é dado por:

$$
s(x, y, w)=\left[m(x, y) \times \operatorname{sinc}\left(\frac{w-w_{\mathrm{cf}}(x, y)}{\delta w}\right)\right] * \psi(x, y),
$$

em que $m(x, y)$ é o mapa de densidade de spins, $w_{\text {cf }}(x, y)$ é o mapa de velocidades, $\delta w$ é a resolução da velocidade e $\psi(x, y)$ é a função de espalhamento de ponto associada à cobertura da trajetória no espaço $k_{x}-k_{y}$ usadas nas aquisições de CVF. A Eq. (5.1) forma 
a base do procedimento que será utilizado para extrair o mapa de velocidades em alta resolução espacial a partir do conjunto de dados adquiridos por CVF de baixa resolução espacial.

Tendo em vista que o modelo do sinal da CVF é descrito pela Eq. (5.1), então, se os efeitos do borramento gerados pela função de borramento $\psi(x, y)$ forem minimizados teremos uma aproximação de $s(x, y, w)$ com melhor resolução espacial:

$$
\tilde{s}(x, y, w) \approx\left[m(x, y) \times \operatorname{sinc}\left(\frac{w-w_{\mathrm{cf}}(x, y)}{\delta w}\right)\right] .
$$

Propomos a deconvolução da distribuição adquiridas por CVF com trajetórias em espiral no espaço $k$, usando a função de espalhamento de ponto associada a essa trajetória, $\psi(x, y)=$ jinc $\left(\sqrt{x^{2}+y^{2}} / \delta r\right)$, em que $\delta r$ é a resolução espacial dos dados de CVF. Para outros tipos de trajetórias, o procedimento é análogo, bastando apenas usar a função de espalhamento de ponto correspondente.

Para estimarmos o mapa de velocidade, comparamos a estimativa obtida para a distribuição de velocidade, $\tilde{s}(x, y, w)$, com uma função sinc, que modela o borramento sobre a dimensão da velocidade, devido à cobertura finita (truncamento) do espaço $k_{w}$. Um mapa de densidade de spins de alta resolução espacial será necessário nesta etapa.

Os detalhes do algoritmo de deconvolução utilizado e do método proposto para estimativa do mapa de velocidades a partir $\tilde{s}(x, y, w)$ serão detalhados nas seções a seguir.

\subsubsection{Algoritmo de Deconvolução Não-Cega}

Considere uma imagem $\mathbf{D}$, com $N$ pixels, obtida através da filtragem da imagem original I por uma função de espalhamento de ponto (FEP) $\psi$ e degradada por um ruído aditivo $\varepsilon$, i.e.:

$$
\mathbf{D}=\mathbf{I} * \psi+\varepsilon
$$

em que * é o operador de convolução bidimensional. Dada, então, uma imagem degradada D, a FEP $\psi$, e o ruído $\varepsilon$, a recuperação da imagem original I nem sempre é uma tarefa simples. Tendo em vista que a Eq. (5.3) pode ser escrita na forma de um sistema linear:

$$
\mathbf{D}=\Psi \mathbf{I}+\varepsilon,
$$


em que $\boldsymbol{\Psi}$ é uma matriz quadrada $N \times N$ (associada à função de espalhamento de ponto $\psi$ ) e a imagem I é escrita na forma de um vetor coluna $N \times 1$, então, a simples inversão desse sistema linear pode levar a amplificação dos níveis de ruído contidos em $\varepsilon$ [79].

Este é um problema inverso, conhecido como deconvolução não-cega, e pertence à classe dos problemas mal-postos. Matematicamente um problema é dito mal-posto quando não se satisfaz uma das três seguintes condições [80]:

- existe solução para o problema;

- a solução do problema é única;

- a solução do problema possui dependência contínua (suave) com dados de entrada.

Não é difícil mostrar que problemas de deconvolução em geral não possuem única solução, mesmo na ausência de ruído [81]. Além disso, como a busca por soluções pode levar a amplificação dos níveis de ruído, é natural a utilização de regularizações, através de funções penalidade $|\cdot|^{\alpha}$, que agem sobre conjuntos de filtros $f_{1}, \cdots, f_{j}$ aplicados a imagem $\mathbf{I}$ e controladas por um parâmetro $\lambda$, que regulam a intensidade dessa regularização [79, 82].

Existem diversos algoritmos eficientes e técnicas que podem ser usadas para determinar a melhor aproximação de I que satisfaz a Eq. (5.3). Aqui está sendo usado o algoritmo de deconvolução recentemente proposto por Krishnan \& Fergus [82]. De um ponto de vista estocástico, esse método procura a estimativa do máximo a posteriori para I, i.e., pelo teorema de Bayes tem-se que:

$$
p(\mathbf{I} \mid \mathbf{D}, \Psi) \propto p(\mathbf{D} \mid \mathbf{I}, \Psi) p(\mathbf{I})
$$

e o interesse é determinar o máximo da função $p(\mathbf{I} \mid \mathbf{D}, \Psi)$. Aqui, o primeiro termo é uma distribuição Gaussiana de média nula e o segundo termo é uma imagem a priori com distribuição hiper-Laplaciana $\left(p(z) \propto e^{-k|z|^{\alpha}}\right)$. Maximizar $p(\mathbf{I} \mid \mathbf{D}, \boldsymbol{\Psi})$ é equivalente a minimizar a função de custo $-\ln p(\mathbf{I} \mid \mathbf{D}, \boldsymbol{\Psi})$, isto é, resolver:

$$
\min _{\mathbf{I}} \sum_{i=1}^{N}\left(\frac{\lambda}{2}(\mathbf{I} * \mathbf{\Psi}-\mathbf{D})_{i}^{2}+\left|\left(\mathbf{I} * f_{1}\right)_{i}\right|^{\alpha}+\left|\left(\mathbf{I} * f_{2}\right)_{i}\right|^{\alpha}\right)
$$

em que $i$ é o índice do pixel e $f_{1}=\left[\begin{array}{ll}1-1\end{array}\right]$ e $f_{2}=[1-1]^{T}$ são os filtros de primeira derivada. Vale observar que é possível a utilização de outros tipos de filtros, como adaptativos [83] 
ou de derivadas de ordens mais altas.

Usando o método da penalidade semi-quadrática [84], é possível introduzir uma variável auxiliar $\mathbf{g}=\left[\begin{array}{ll}g_{i}^{1} & g_{i}^{2}\end{array}\right]$ (os números $j$ em $g_{i}^{j}$ são índices, não potências) em cada pixel $i$ que permite mover os termos $\left(\mathbf{I} * f_{j}\right)_{i}$ para fora da expressão $|\cdot|^{\alpha}$, fornecendo uma nova função de custo:

$$
\min _{\mathbf{I}, \mathbf{g}} \sum_{i=1}^{N}\left[\frac{\lambda}{2}(\mathbf{I} * \mathbf{\Psi}-\mathbf{D})_{i}^{2}+\frac{\beta}{2}\left(\left\|\left(\mathbf{I} * f_{1}\right)_{i}-g_{i}^{1}\right\|_{2}^{2}+\left\|\left(\mathbf{I} * f_{2}\right)_{i}-g_{i}^{2}\right\|_{2}^{2}\right)+\left|g_{i}^{1}\right|^{\alpha}+\left|g_{i}^{2}\right|^{\alpha}\right]
$$

em que $\beta$ é um peso que irá variar durante a otimização. Quando $\beta \rightarrow \infty$, a solução da Eq. (5.7) converge para a Eq. (5.6] [84. A minimização da Eq. (5.7) para um $\beta$ fixo é feita alternando dois passos: no primeiro passo, resolve-se a Eq. (5.7) para I, dado um valor fixado para g e, em um segundo passo, determina-se a solução de uma equação auxilar para g com I fixado [82].

A utilização desse algoritmo de deconvolução apresenta algumas vantagens. Uma delas está relacionada a velocidade com que se obtem a imagem filtrada e a outra está relacionada com o tratamento da informação a priori. Caso seja conhecido algo da estatística da imagem que se deseja obter, I, é possível modificar o algoritmo para obter a melhor filtragem da imagem degradada D possível. O conhecimento da informação a priori está diretamente relacionado ao parâmetro $\alpha$. Neste trabalho, foram utilizados valores de $\alpha$ e $\lambda$ testados que apresentaram o menor erro possível na estimação da imagem filtrada.

\subsubsection{Estimando o Mapa de Velocidades}

Assumindo que a imagem obtida pelo método eCVF pode ser escrita na forma da Eq. (5.3), então, após a deconvolução dessa imagem pela função de borramento $\psi(r)=$ jinc $(r / \delta r)$, devemos obter uma distribuição com alta resolução espacial dada aproximadamente por:

$$
\tilde{s}(x, y, w) \approx m(x, y) \times \operatorname{sinc}\left(\frac{w-w_{\mathrm{cf}}(x, y)}{\delta w}\right) .
$$

A partir da distribuição $\tilde{s}(x, y, w)$ a velocidade $\bar{w}_{\text {cvf }}$ em uma dada coordenada espacial $\left(x_{o}, y_{o}\right)$ poderia ser estimada de uma forma ingênua utilizando a seguinte maximização:

$$
\bar{w}_{\mathrm{cvf}}\left(x_{o}, y_{o}\right)=\underset{\omega \in\left\{w_{k}\right\}_{k=1}^{K}}{\arg \max }\left\{\left\|\tilde{s}\left(x_{o}, y_{o}, \omega\right)\right\|\right\}
$$


em que $w_{k}=\left(\frac{k-1}{K}-\frac{1}{2}\right) \cdot \mathrm{FOV}_{w}$ é a coordenada do $k$-ésimo ponto (ao longo do eixo $w$ ) na grade em que $\tilde{s}(x, y, w)$ foi calculado, $\mathrm{FOV}_{w}$ é o comprimento dessa grade e $K$ é o número de pontos. Essa é uma forma ingênua de se obter o mapa de velocidades pois a velocidade correta na coordenada espacial $\left(x_{o}, y_{0}\right)$ é dada pelo número real $w$ tal que sinc $\left[\left(w-w_{\mathrm{cf}}\left(x_{o}, y_{o}\right)\right) / \delta w\right]=1$. Desta forma, se a velocidade real $w_{\mathrm{cf}}\left(x_{o}, y_{o}\right)$ estiver, por exemplo, no ponto médio do intervalo $\left[w_{j}, w_{j+1}\right]$ para um $j$ qualquer $(1<j<K-1)$, então a velocidade aproximada $\bar{w}_{\text {cvf }}$ terá um erro de exatamente $\left(w_{j+1}-w_{j}\right) / 2$. Assim, quanto mais grosseira for a grade do eixo $(w)$, isto é, quanto maior for $\mathrm{FOV}_{w} / K$, maior será o erro associado à velocidade recuperada $\bar{w}_{\mathrm{cvf}}$.

Com o intuito de se obter uma melhor aproximação para o mapa de velocidades, propomos a seguinte minimização. Supondo que se possua um mapa de magnitude, $\tilde{m}(x, y)$, de alta resolução espacial (que pode ser medido rapidamente com uma aquisição à parte), a velocidade $\hat{w}_{\text {cvf }}$ em uma dada coordenada espacial $\left(x_{o}, y_{o}\right)$ pode ser estimada a partir da distribuição $\tilde{s}(x, y, w)$ resolvendo o seguinte problema de minimização:

$$
\hat{w}_{\mathrm{cvf}}\left(x_{o}, y_{o}\right)=\underset{\omega}{\arg \min }\left\|\frac{\tilde{s}\left(x_{o}, y_{o}, w_{k}\right)}{\tilde{m}\left(x_{o}, y_{o}\right)}-\operatorname{sinc}\left(\frac{w_{k}-\omega}{\delta w}\right)\right\|_{2},
$$

para $\tilde{m}\left(x_{o}, y_{o}\right) \neq 0$, caso contrário $\hat{w}_{\mathrm{cvf}}\left(x_{o}, y_{o}\right)=0$. Com essa minimização, espera-se um resultado qualitativo e quantitativo melhor que a solução obtida pela maximização dada pela Eq. (5.9).

\subsection{Experimentos}

Os conjuntos de dados de contraste de fase utilizados neste trabalho (in vivo e phantom) são dados por um campo de velocidades na forma

$$
\vec{\nu}_{\mathrm{cf}}(x, y, z, t)=u_{\mathrm{cf}}(x, y, z, t) \mathbf{i}+v_{\mathrm{cf}}(x, y, z, t) \mathbf{j}+w_{\mathrm{cf}}(x, y, z, t) \mathbf{k} .
$$

Assim, para simularmos uma distribuição de velocidade com codificação de velocidade em Fourier com aquisição em espiral (eCVF), inicialmente será fixada uma fase $t=t_{o}$ e também uma seção transversal $z=z_{o}$. A direção de velocidade na direção $z$ foi escolhida para ser utilizada na distribuição com eCVF simulada por ser a direção principal do escoamento. Dessa forma, a distribuição de velocidade eCVF simulada associada a cada seção transversal 
$z_{o}$ será dada pela equação:

$$
s\left(x, y, z_{o}, w, t_{o}\right)=\left[m\left(x, y, z_{o}, t_{o}\right) \times \operatorname{sinc}\left(\frac{w-w_{\mathrm{cf}}\left(x, y, z_{0}, t_{o}\right)}{\delta w}\right)\right] * \psi(x, y),
$$

em que $m\left(x, y, z_{o}, t_{o}\right)$ é o mapa de densidade de spins do phantom no corte $z_{o}$ e para o instante $t=t_{o}$. Tendo em vista que estamos simulando um conjunto de dados em espiral, a função de espalhamento de ponto neste caso será dada pela função $\psi(x, y)=$ jinc $\left(\sqrt{x^{2}+y^{2}} / \delta r\right)$, em que $\delta r$ é a resolução espacial da distribuição eCVF e a função jinc $(\xi)$ é definida como jinc $(\xi)=J_{1}(\pi \xi) / 2 \xi$, sendo $J_{1}(\xi)$ a função de Bessel de primeira espécie [58].

Foram, então, simuladas três CVF com aquisições em espiral, um para cada valor de $\delta r(1,0 ; 1,5$ e 2,0 mm). O conjunto de dados de contraste de fase do phantom possui resolução espacial nos cortes transversais dada por $0,5 \times 0,5 \mathrm{~mm}^{2}$. Para a resolução da velocidade, foi escolhido o valor $\delta w=10 \mathrm{~cm} / \mathrm{s}$, num campo de visão $\mathrm{FOV}_{w}=120 \mathrm{~cm} / \mathrm{s}$. O instante $t_{o}$ escolhido para determinarmos as distribuições simuladas foi o correspondente ao $4^{\circ}$ quadro temporal (fase 4 ), pois este é o que está mais próximo ao pico do escoamento.

Em seguida, mapas de velocidade foram reconstruídos para cada uma das três distribuições eCVF simuladas, utilizando o algoritmo de deconvolução e a abordagem por minimização propostos nas Subseções 5.1.1 e 5.1.2, respectivamente. Nos parâmetros de obtenção dos mapas $\hat{w}_{\text {cvf }}$, dados pela Eq. (5.6), foram utilizados os valores $\lambda=5 \times 10^{2} \mathrm{e}$ $\alpha=5 / 3$ para a resolução $\delta r=1,0 \mathrm{~mm}$; os valores $\lambda=10^{3}$ e $\alpha=5 / 3$ para a resolução espacial $\delta r=1,5 \mathrm{~mm}$; e os valores $\lambda=10$ e $\alpha=5 / 3$ para a resolução $\delta r=2,0 \mathrm{~mm}$. Esses valores foram escolhidos de forma que o mapa de velocidades tenha o menor erro possível.

Finalmente, cada um dos mapas de velocidade $\hat{w}_{\text {cvf }}$ obtidos da distribuição eCVF simulada foi utilizado para guiar uma solução computacional DFC do escoamento do fluido mimetizador de sangue no phantom, como realizado no Capítulo 4 usando mapas obtidos por contraste de fase, $w_{\mathrm{cf}}$. As soluções obtidas, então, foram comparadas qualitativamente e quantitativamente com o mapa de velocidade medido por contraste de fase do phantom, com a solução computacional pura - i.e., obtida apenas com a dinâmica dos fluidos computacional - e com dois campos de velocidade guiados pela componente $w_{\text {cf }}$ : um obtido usando um único NEX da aquisição do contraste de fase (que corresponde a aproximadamente o mesmo tempo de aquisição de uma distribuição eCVF com resolução espacial $\delta r$ 
$=1 \mathrm{~mm}$ ), e o outro obtido usando todos os 9 NEX (isto é, 9 vezes o tempo de aquisição).

Os parâmetros utilizados nas simulações computacionais são os mesmos do Capítulo 4, a saber: $\mu=0,005 \mathrm{~Pa} \cdot \mathrm{s}, \rho=1100 \mathrm{~kg} / \mathrm{m}^{3}$, malha Cartesiana com resolução espacial $0,5 \times 0,5 \times 1,0 \mathrm{~mm}^{3}$ e passo de tempo computacional $\delta t=0,1 \mathrm{~ms}$. As simulações guiadas com o mapa de velocidade $\hat{w}_{\text {cvf }}$ reconstruído da distribuição eCVF precisaram em média de 250 iterações até a convergência considerando, o valor $\varepsilon=10^{-3}$ dado na Eq. 4.4).

\subsubsection{Resultados}

Na Fig. 5.1 podemos ver os efeitos do borramento espacial para cada uma das resoluções escolhidas, em três diferentes cortes transversais da bifurcação carotídea do phantom (linhas (i)). Há de se esperar que, quanto maior for o borramento, menos preciso será o mapa de velocidades recuperado através das Eq. (5.9) e Eq. 5.10). É possível verificar esse comportamento qualitativamente comparando os mapas de velocidade apresentados nas linhas (ii) e (iii). Nas linhas (ii) são mostrados os mapa de velocidades obtidos com maximização dada pela Eq. (5.9), enquanto que na Fig. 5.1(iii) são mostrados aqueles obtidos pela minimização proposta dada pela Eq. 5.10. É clara a diferença entre as formas de se recuperar o mapa de velocidades a partir das distribuições com eCVF. Qualitativamente, percebe-se que a minimização proposta na Eq. (5.10) proporciona um resultado qualitativo mais próximo do mapa de velocidades medido com contraste de fase do que o outro método.

$\mathrm{Na}$ análise qualitativa dos erros absolutos, dados na Fig. 5.2, verifica-se novamente que, quanto mais grosseira for a resolução espacial, pior será a reconstrução do mapa de velocidades a partir das distribuições com eCVF. Nas linhas (i) e (ii) são mostrados mapas de diferença entre os dois métodos de reconstrução do mapa de velocidades a partir da distribuição eCVF, dados pelas Eq. (5.9) e Eq. (5.10), respectivamente. Fica claro, qualitativamente, que a minimização proposta na Eq. 5.10 de fato proporciona um melhor resultado que a maximização simplória dada pela Eq. $(5.9)$.

Quantitativamente, são apresentados na Tab. 5.1, a relação sinal-erro e o erro absoluto médio (EAM) para os mapas de velocidade reconstruídos, $\bar{w}_{\text {cvf }}$ e $\hat{w}_{\text {cvf }}$, dados pelas Eq. 5.9 e Eq. (5.10), respectivamente. Conforme resultado esperado da análise qualitativa, vêse que a minimização proposta na Eq. 5.10 resulta em EAM menores e SER maiores que a maximização simplória (Eq. (5.9)). O mapa de velocidades $\hat{w}_{\text {cvf }}$ tem um ganho na 
SER, comparado com o mapa $\bar{w}_{\text {cvf }}$, de 0,62 dB; 1,19 dB e 1,19 dB para as resoluções $\delta r=1,0 ; 1,5$ e 2,0 mm, respectivamente. Consequentemente, o EAM é menor para o mapa de velocidades $\hat{w}_{\text {cvf }}$, como também pode ser apreciado na Tab. 5.1. Como foi observado qualitativamente na Fig. 5.1 o EAM, aumenta de acordo com o aumento do tamanho do voxel. Em comparação com o erro absoluto médio do mapa de velocidade $\hat{w}_{\text {cvf }}$ para $\delta r=1,0 \mathrm{~mm}$, observa-se um aumento de $1,28 \mathrm{~cm} / \mathrm{s}$ e $2,71 \mathrm{~cm} / \mathrm{s}$ no erro para os valores de resolução espacial $\delta r=1,5 \mathrm{~mm}$ e $\delta r=2,0 \mathrm{~mm}$, respectivamente. Levando em consideração que a velocidade máxima atingida pela componente $w_{\mathrm{cf}}$ é de $48,12 \mathrm{~cm} / \mathrm{s}$, então os erros médios representam uma diferença de $5 \%, 7 \%$ e 10\%, para as resoluções espaciais $\delta r=1,0$; 1,5 e 2,0 mm, respectivamente. Esses resultados sugerem que é possível extrair mapas de velocidade das distribuições de eCVF com boa qualidade, os quais podem ser úteis para guiar simulações computacionais, como demonstrado a seguir.

Na Figura 5.3 são mostradas comparações qualitativas entre as componentes de velocidade $u_{\mathrm{cf}}, v_{\mathrm{cf}}$ e $w_{\mathrm{cf}}$, medidas usando ressonância magnética por contraste de fase, para três cortes transversais da "artéria carótida" do phantom, e: as soluções de DFC produzidas com influência de mapas de velocidades recuperados das distribuições de velocidade eCVF, $\hat{w}_{\mathrm{cvf}}$, para as três resoluções espaciais $(\delta r=1,0 ; 1,5$ e 2,0 mm). Percebe-se uma semelhança qualitativa entre os mapas de velocidade simulados pela DFC, nas três direções, e aqueles adquiridos por contraste de fase. Além disso, nota-se que os mapas simulados pela DFC se assemelham entre si. É um comportamento esperado, tendo em vista que os mapas recuperados das distribuições com eCVF são próximos entre si, logo as soluções computacionais devem se parecer.

Os campos de velocidade em todos os experimentos simulados e o medido com contraste de fase são apresentados na Fig. 5.4. Em uma comparação qualitativa entre a medida do contraste de fase $(\mathrm{CF})$ e os demais campos simulados (DFC + 1D CF e DFC + eCVF) percebemos que as soluções obtidas utilizando as distribuições eCVF proporcionam um resultado visivelmente mais parecido com o campo de velocidade CF que a simulação computacional pura (DFC). Também são mais parecidos que a solução obtida para a simulação guiada por uma componente do constraste de fase ruidoso (DFC $+1 \mathrm{D} C F, 1 \mathrm{NEX}$ ), que apresenta tempo de aquisição similar a distribuição eCVF. Além disso, fica claro que a solução DFC + eCVF para $\delta r=1,0 \mathrm{~mm}$ se assemelha bastante com a solução guiada por uma componente do contraste de fase com baixo ruído (DFC + 1D CF, 9 NEX). Isso é um bom 
resultado, pois a aquisição de uma distribuição eCVF com resolução $\delta r=1,0 \mathrm{~mm}$ seria nove vezes mais rápida que a aquisição de uma componente de velocidade por contraste de fase com resolução espacial $\delta r=0,5 \mathrm{~mm}$ com $9 \mathrm{NEX}$.

Finalmente, na Tabela 5.2 são apresentados os resultados do cálculo da relação sinalerro (Eqs. 4.15) a 4.18) relativos ao contraste de fase medido, para as várias soluções numéricas mostradas na Fig. 5.4. De imediato, verifica-se que as três soluções computacionais geradas a partir do mapa de velocidades recuperado da distribuição eCVF possuem, para o campo de velocidades $\vec{\nu}$, uma relação sinal-erro maior que a da solução computacional pura. Há um ganho de 1,45 dB, 1,31 dB e 0,97 dB com relação ao SER da solução computacional pura para os três valores da resolução espacial $\delta r=1,0 ; 1,5 ;$ e 2,0 mm, respectivamente. Além disso, quando se compara as simulações obtidas pelas distribuições eCVF à simulação guiada por contraste de fase com 1 NEX, verifica-se que há um ganho considerável: $3,61 \mathrm{~dB}$ para $\delta r=1,0 \mathrm{~mm}$ (tempo de aquisição similar); 3,47 dB para para $\delta r=1,5 \mathrm{~mm}$; e $3,13 \mathrm{~dB}$ para $\delta r=2,0 \mathrm{~mm}$ (três vezes mais rápido que a aquisição de 1 NEX do contraste de fase). Finalmente, comparando a simulação guiada por contraste de fase com 9 NEX (que requer um longo tempo de aquisição, aproximadamente 2 horas), a SER foi apenas $0,36 \mathrm{~dB}$ superior à SER da simulação guiada pelo eCVF com $\delta r=1,0 \mathrm{~mm}$ (possui aquisição nove vezes mais rápida); 0,50 dB para $\delta r=1,5 \mathrm{~mm}$; e 0,84 $\mathrm{dB}$ superior para $\delta r=2,0 \mathrm{~mm}$ (possui aquisição 27 vezes mais rápida).

Para as três simulações guiadas por eCVF houve um ganho na SER para as componentes de velocidade $u$ e $w$ comparado com a solução computacional pura. Já para a componente de velocidade $v$, houve uma ligeira perda de SER. Esse comportamento pode ser o efeito positvo da redução de ruído, pois as velocidades ao longo do eixo y são extremamente baixas (valores de $v$ são próximos de zero em todo o domínio) lembrando que a energia total da componente $w_{\mathrm{cf}}$ é $15,7 \mathrm{~dB}$ maior que a da componente $v_{\mathrm{cf}}$. De todo modo, o ganho da SER para as componentes $u$ e $w$ compensam esse comportamento contrário da componente $v$. Além disso, houve um ligeiro ganho na SER das componentes $w$ para as três simulações computacionais guiadas por eCVF (Tabela 5.2) se comparadas com o SER do mapa de velocidade recuperado das distribuições com eCVF, $\hat{w}_{\text {cvf }}$ (Tabela 5.1). O ganho foi de 0,20 dB, 0,14 dB e 0,40 dB, respectivamente para as resoluções $\delta r=1,0 ; 1,5$ e 2, $0 \mathrm{~mm}$. 


\subsection{Discussão e Conclusões}

Os resultados apresentados neste capítulo mostram que é possível a utilização de distribuições de velocidade com codificação de velocidade em Fourier para guiar soluções computacionais de DFC. As distribuições de velocidade foram geradas a partir das equações que definem o sinal de uma distribuição de velocidade medida com CVF (Eq. 2.19) e Eq. (2.20) ) e, utilizando o método apresentado, os mapas de velocidade na direção z foram recuperados. Foram simuladas distribuições de velocidade eCVF para somente a componente $w$ pois, em uma situação clínica o tempo para se adquirir uma distribuição espaço-velocidade para três componentes de velocidade, $s(x, y, z, u, v, w)$, seria absolutamente impraticável. Considerando ainda a dimensão temporal, seria necessário adquirir, armazenar e processar uma matriz de 7 dimensões, o que também resulta em um custo computacional bastante considerável.

De todo modo, a aquisição de uma distribuição de velocidade com eCVF resolvida somente para a componente $w$ além de ser mais rápida que a aquisição de apenas uma dimensão utilizando o contraste de fase, proporciona uma maior relação sinal-ruído. Um conjunto de dados eCVF com 1,0 mm de resolução espacial pode ser adquirido no mesmo tempo que 1 NEX de um campo de velocidades medido por contraste de fase com 0,5 mm de resolução espacial, com os mesmos parâmetros utilizados neste trabalho; entretanto o conjunto de dados com codificação de velocidade em Fourier possui $23 \mathrm{~dB}$ a mais de SNR que o conjunto de dados do contraste de fase (para um conjunto de dados eCVF com 2,0 mm de resolução espacial, a aquisição seria 3 vezes mais rápida que o contraste de fase, e se teria ainda $8 \mathrm{~dB}$ a mais de SNR que o conjunto de dados do contraste de fase). Além disso, foi mostrado no Capítulo 4 que a utilização de RM com apenas uma direção de velocidade para guiar as soluções de DFC, para o modelo físico escolhido neste trabalho, proporciona resultados melhores do que as simulações computacionais puras, i.e., com a mínima influência dos dados de ressonância magnética.

Qualitativamente e quantitativamente, foi mostrado neste capítulo que a recuperação de mapas de velocidade a partir de dados com eCVF é eficiente, mesmo quando se tem um borramento mais acentuado. Também foi mostrado que mesmo o mapa de velocidades recuperado com a pior relação sinal-erro ainda produz simulações computacionais mais próximas dos dados medidos por contraste de fase que a solução computacional pura. Além 
disso, quando comparadas as simulações guiadas pelas distribuições eCVF às simulações guiadas pelo contraste de fase utilizando uma componente de velocidade e reconstruída com apenas 1 NEX, obtivemos um resultado qualitativo e quantitativo consideravelmente melhor para as soluções guiadas pelas distribuições eCVF. Tendo em vista que, para os parâmetros escolhidos, a aquisição de uma distribuição eCVF pode ser até três vezes mais rápida que 1 NEX do contraste de fase, este é um resultado importante. Na última análise qualitativa e quantitativa realizada, vimos que a simulação computacional guiada por CF com somente uma componente de velocidade reconstruída utilizando os 9 NEX é qualitativamente similar aos campos de velocidade simulados obtidos utilizando as distribuições eCVF e, quantitativamente, proporciona, no melhor caso, somente 0,84 dB a mais de SER que a simulação guiada pela distribuição eCVF. Por outro lado, neste caso a aquisição da distribuição eCVF seria 27 vezes mais rápida que a do mesmo contraste de fase utilizado. Isso seria aproximadamente 5 minutos, o que contrasta de forma considerável com as 2 horas necessárias para a aquisição por contraste de fase dos 9 NEX de uma componente de velocidade.

Finalmente, levando em consideração a rápida convergência do algoritmo computacional com a rápida aquisição das distribuições de velocidade adquiridas com eCVF, os resultados apresentados neste capítulo indicam que o método que combina dados de eCVF e a DFC pode ser utilizado no ambiente clínico. Além disso, com os resultados apresentados mostram que esse método pode ser utilizado como uma técnica para redução do tempo de 
aquisição de campos de velocidade medidos por RM.

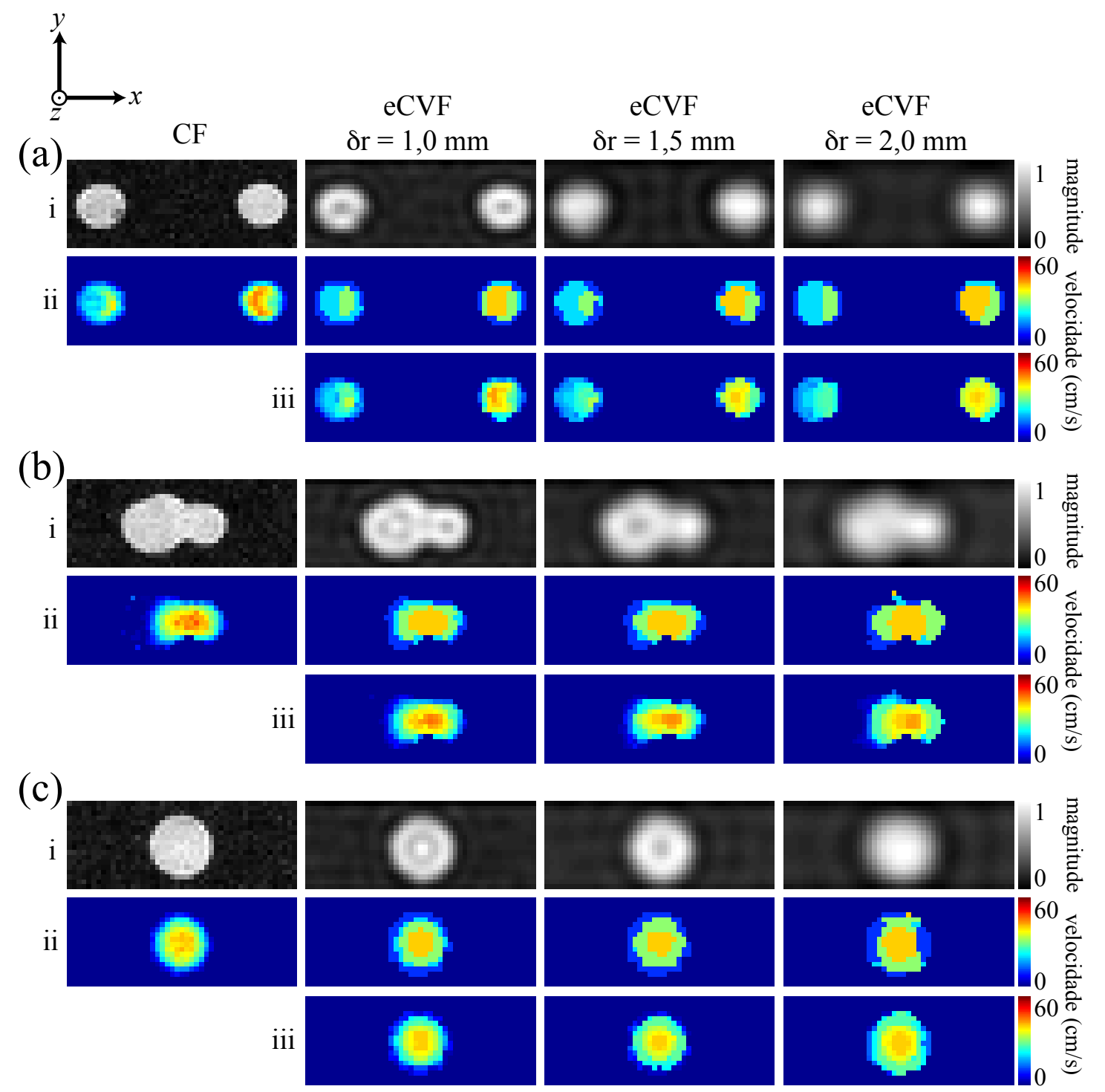

Figura 5.1: Avaliação qualitativa da obtenção de mapas de velocidade a partir de distribuições de eCVF: (i) seções transversais do mapa de densidade de spins para os diferentes tipos de aquisição (CF e eCVF); (ii) mapa de velocidade (na direção $z$ ) obtido com o contraste de fase (CF) e mapas recuperados das distribuições eCVF para os diferentes valores da resolução espacial $(\delta r=1,0 ; 1,5$ e 2,0 mm) com a maximização dada pela Eq. (5.9); e (iii) mapas de velocidade recuperados das distribuições eCVF para os diferentes valores da resolução espacial com a minimização dada pela Eq. (5.10). São mostrados três diferentes cortes da "artéria carótida" do phantom, a saber: (c) carótida comum, (b) bifurcação da carótida e (a) carótida bifurcada. 

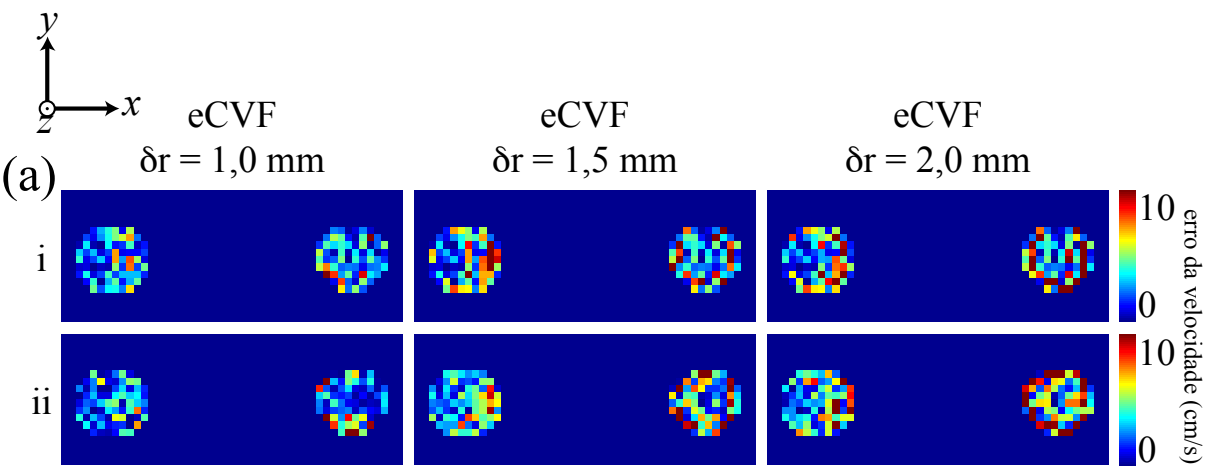

(b)
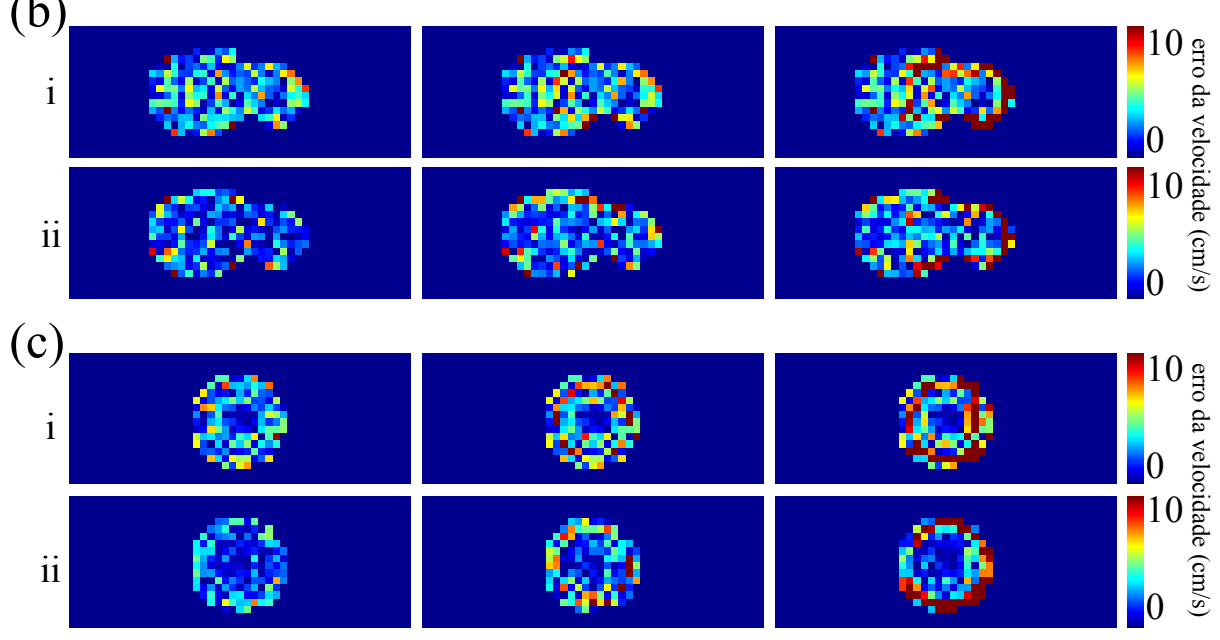

Figura 5.2: Erro absoluto entre o mapa de velocidades $w_{\mathrm{cf}}$ medido com contraste de fase e os mapas de velocidade $\bar{w}_{\text {cvf }}$ e $\hat{w}_{\text {cvf }}$ recuperados das distribuições com eCVF (mostrados na Fig. 5.1). (i) utilizando a maximização dada pela Eq. (5.9); e (ii) utilizando a minimização proposta na Eq. (5.10). São mostrados três diferentes cortes da "artéria carótida" do phantom, a saber: (c) carótida comum, (b) bifurcação da carótida e (a) carótida bifurcada.

\begin{tabular}{ccc} 
eCVF & eCVF & eCVF \\
$\delta r=1,0 \mathrm{~mm}$ & $\delta r=1,5 \mathrm{~mm}$ & $\delta r=2,0 \mathrm{~mm}$ \\
\hline
\end{tabular}

\begin{tabular}{cccc} 
Relação-sinal erro & & \\
\hline $\bar{w}_{\text {cvf }}$ (Eq. $\left.(5.9)\right)$ & $9,96 \mathrm{~dB}$ & $8,53 \mathrm{~dB}$ & $6,73 \mathrm{~dB}$ \\
$\hat{w}_{\text {cvf }}$ (Eq. $(5.10)$ & $10,65 \mathrm{~dB}(\uparrow)$ & $9,09 \mathrm{~dB}(\uparrow)$ & $7,22 \mathrm{~dB}(\uparrow)$ \\
\hline & & \\
\hline \\
Erro absoluto médio \\
\hline $\bar{w}_{\text {cvf }}$ (Eq. $\left.(5.9)\right)$ & $3,16 \mathrm{~cm} / \mathrm{s}$ & $4,17 \mathrm{~cm} / \mathrm{s}$ & $5,45 \mathrm{~cm} / \mathrm{s}$ \\
$\hat{w}_{\text {cvf }}$ (Eq. $(5.10)$ & $2,43 \mathrm{~cm} / \mathrm{s}(\downarrow)$ & $3,72 \mathrm{~cm} / \mathrm{s}(\downarrow)$ & $5,07 \mathrm{~cm} / \mathrm{s}(\downarrow)$ \\
\hline
\end{tabular}

Tabela 5.1: Relação sinal-erro e erro absoluto médio entre o mapa de velocidade medido por contraste de fase na direção $z, w_{\text {cf }}$, do phantom na fase 4 e os mapas de velocidade na direção $z$ recuperados das distribuições de velocidade eCVF, $w_{\mathrm{cvf}}$, para os diferentes valores da resolução espacial $(\delta r=1,0 ; 1,5$ e $2,0 \mathrm{~mm})$. 


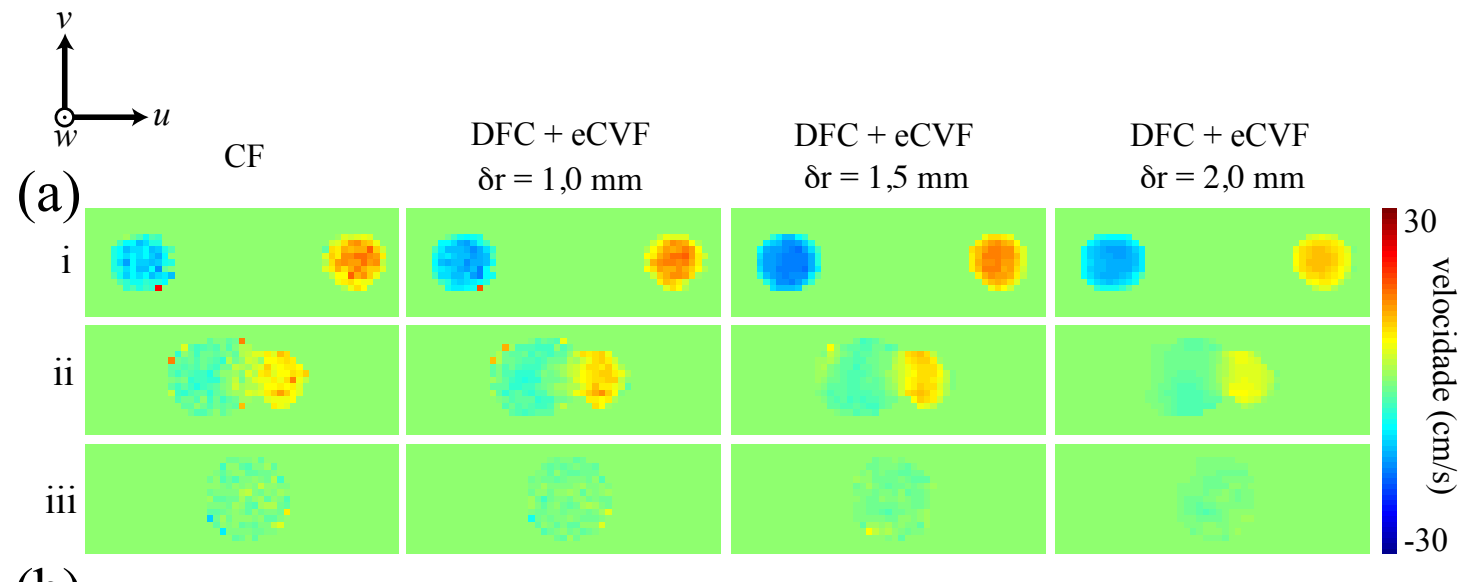

(b)

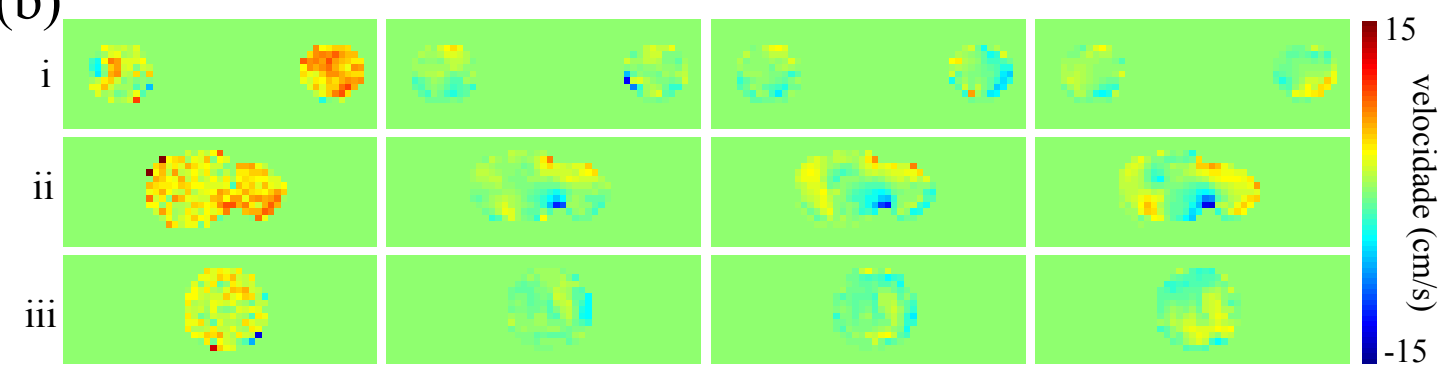

(c)

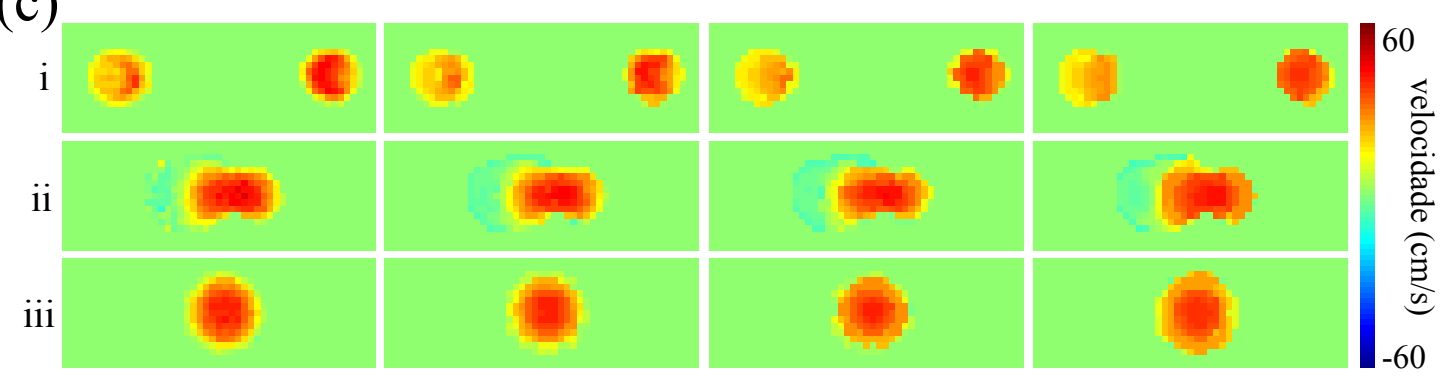

(d)

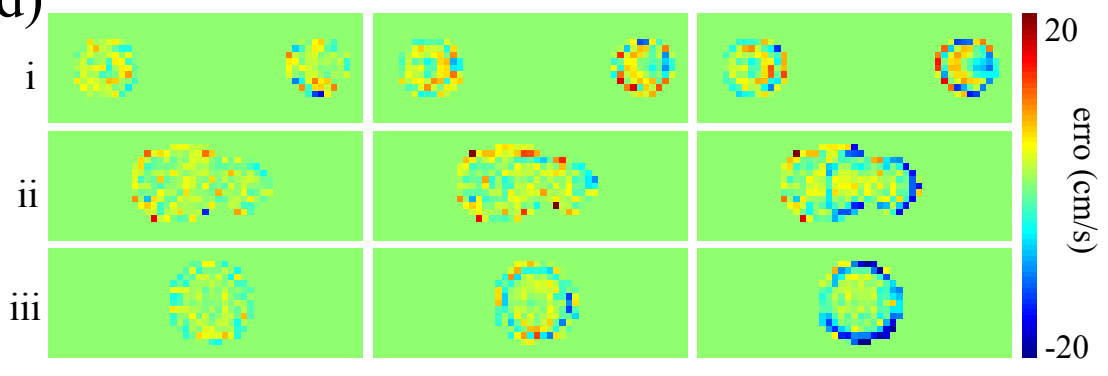

Figura 5.3: Seções transversais das componentes de velocidade: (a) $u$; (b) $v$; (c) $w$; e (d) $w_{\mathrm{cf}}-\hat{w}_{\mathrm{cvf}}$. As linhas representam três diferentes cortes da "artéria carótida" do phantom, a saber: (iii) carótida comum, (ii) bifurcação da carótida e (i) carótida bifurcada; e as colunas representam os diferentes experimentos: contraste de fase $(\mathrm{CF})$ medido; e simulações numéricas guiadas pelas componentes de velocidade recuperadas da distribuição eCVF (DFC + eCVF) para as diferentes resoluções espaciais $(\delta r=1,0 ; 1,5$ e $2,0 \mathrm{~mm})$. 


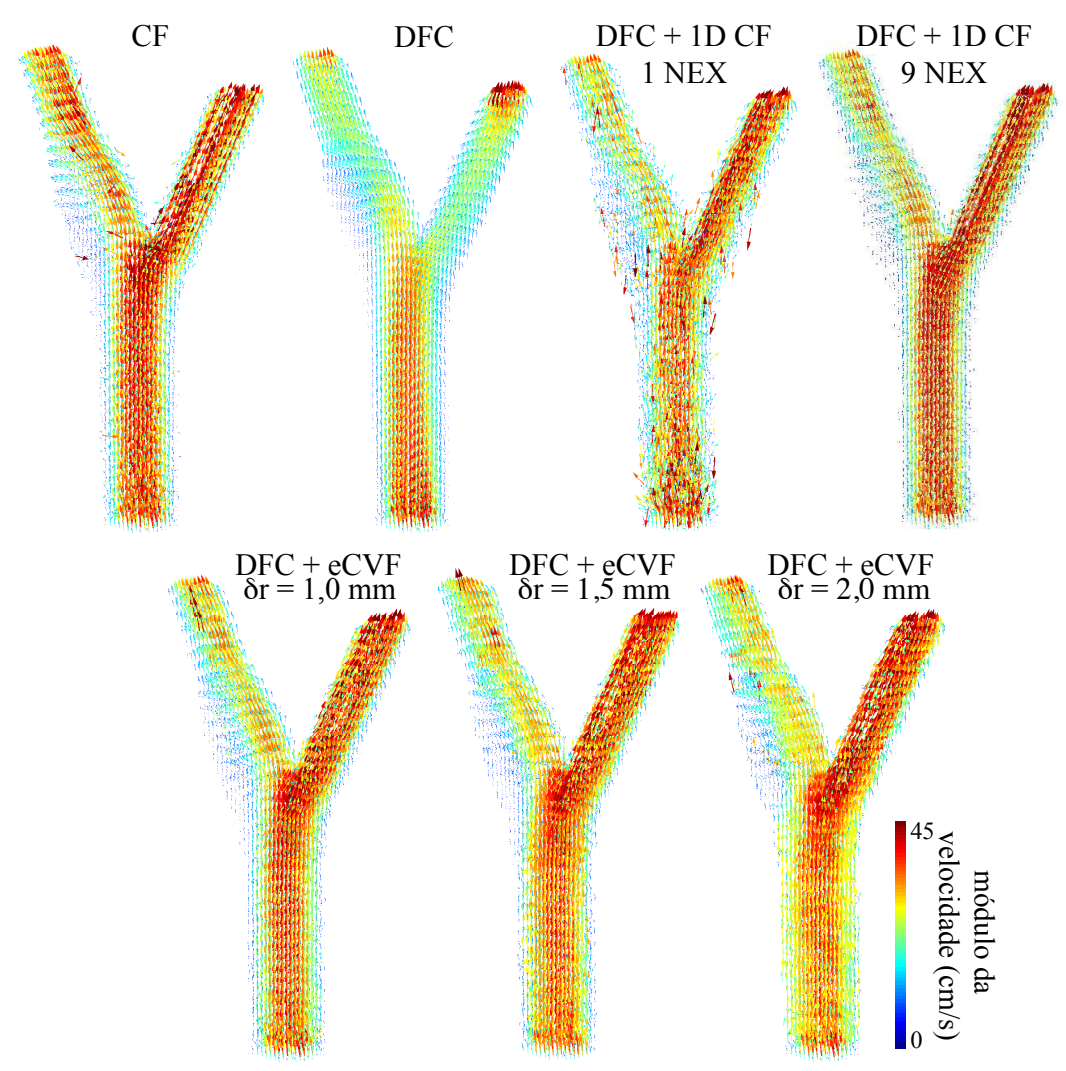

Figura 5.4: Visualização do campo de velocidades no phantom para os diferentes experimentos: contraste de fase (CF) medido; simulação computacional pura (DFC); simulações numéricas guiadas por contraste de fase com somente a componente $w_{\text {cf }}$, reconstruídas com 1 NEX e 9 NEX; e simulações numéricas guiadas pelas componentes de velocidade recuperadas da distribuição eCVF $(\mathrm{DFC}+\mathrm{eCVF})$, para as diferentes resoluções espaciais $(\delta r=1,0 ; 1,5$ e $2,0 \mathrm{~mm})$.

\begin{tabular}{lccc}
\hline & DFC & DFC + 1D CF & DFC + 1D CF \\
& & $1 \mathrm{NEX}$ & $9 \mathrm{NEX}$ \\
\hline $\mathrm{SER}_{u}$ & $2,97 \mathrm{~dB}$ & $2,72 \mathrm{~dB}(\downarrow)$ & $4,16 \mathrm{~dB}(\uparrow)$ \\
$\mathrm{SER}_{v}$ & $-0,25 \mathrm{~dB}$ & $-0,88 \mathrm{~dB}(\downarrow)$ & $-0,30 \mathrm{~dB}(\downarrow)$ \\
$\mathrm{SER}_{w}$ & $5,44 \mathrm{~dB}$ & $6,21 \mathrm{~dB}(\uparrow)$ & $16,53 \mathrm{~dB}(\uparrow \uparrow \uparrow)$ \\
\hline $\mathrm{SER}_{\vec{\nu}}$ & $6,57 \mathrm{~dB}$ & $4.41 \mathrm{~dB}(\downarrow)$ & $8.38 \mathrm{~dB}(\uparrow)$ \\
\hline \multicolumn{4}{c}{} \\
\hline & $\mathbf{D F C}+\mathbf{e C V F}$ & $\mathbf{D F C}+\mathbf{e C V F}$ & $\mathbf{D F C}+\mathbf{e C V F}$ \\
& $\delta r=1,0 \mathrm{~mm}$ & $\delta r=1,5 \mathrm{~mm}$ & $\delta r=2,0 \mathrm{~mm}$ \\
\hline $\mathrm{SER}_{u}$ & $3,92 \mathrm{~dB}(\uparrow)$ & $3,97 \mathrm{~dB}(\uparrow)$ & $4,11 \mathrm{~dB}(\uparrow)$ \\
$\mathrm{SER}_{v}$ & $-0,38 \mathrm{~dB}(\downarrow)$ & $-0,50 \mathrm{~dB}(\downarrow)$ & $-0,55 \mathrm{~dB}(\downarrow)$ \\
$\mathrm{SER}_{w}$ & $10,85 \mathrm{~dB}(\uparrow \uparrow)$ & $9,23 \mathrm{~dB}(\uparrow)$ & $7,62 \mathrm{~dB}(\uparrow)$ \\
\hline $\mathrm{SER}_{\vec{\nu}}$ & $8,02 \mathrm{~dB}(\uparrow)$ & $7,88 \mathrm{~dB}(\uparrow)$ & $7,54 \mathrm{~dB}(\uparrow)$ \\
\hline
\end{tabular}

Tabela 5.2: Relação sinal-erro entre o campo de velocidade medido por contraste de fase, $\vec{\nu}_{\mathrm{cf}}=$ $\left(u_{\mathrm{cf}}, v_{\mathrm{cf}}, w_{\mathrm{cf}}\right)$, do phantom na fase 4 e os campos de velocidade obtidos através das simulações numéricas guiadas por contraste de fase com somente a componente $w_{\text {cf }}$ e reconstruídas com 1 NEX ou 9 NEX; e as simulações numéricas guiadas pelos mapas de velocidade recuperados das distribuições de velocidade eCVF (DFC + eCVF) para as diferentes resoluções espaciais $(\delta r=1,0$; 1,5 e $2,0 \mathrm{~mm}$ ). Os valores obtidos estão sendo comparados com a solução computacional pura na fase 4 do phantom (DFC), mostrada no Capítulo 4. 


\section{Capítulo 6}

\section{Conclusões e Trabalhos Futuros}

Neste trabalho focamos no problema de relacionar dados de velocidade adquiridos por ressonância magnética as simulações computacionais de escoamento baseadas em modelos físicos da dinâmica dos fluidos. Nos primeiros capítulos apresentamos a motivação deste trabalho, uma revisão bibliográfica do imageamento por ressonância magnética e também sobre o imageamento de fluxo por ressonância magnética. Foi feita também uma revisão bibliográfica sobre um método computacional para determinar as soluções numéricas das equações do movimento da dinâmica dos fluidos em malhas Cartesianas. O algoritmo para determinar as soluções das equações do movimento com acoplamento entre velocidade e pressão, SIMPLER (Semi-Implicit Method for Pressure Linked Equations Revised), foi deduzido para o caso bidimensional e, a seguir, testado e validado utilizando resultados obtidos na literatura para o problema clássico do escoamento de um fluido em uma cavidade. Além disso, foi implementada uma versão tridimensional do algoritmo com o intuito de realizar as simulações computacionais guiadas por dados adquiridos com ressonância magnética.

Nos demais capítulos, foram apresentadas três contribuições originais. Primeiramente é mostrado um método capaz de produzir um melhoramento dos dados de contraste de fase usando modelos físicos da dinâmica dos fluidos. O método é baseado na inclusão de novas linhas e colunas, com os dados de contraste de fase, no sistema linear que surge após a discretização das equações do movimento que modelam o escoamento do sangue (ou do fluido mimetizador de sangue, no caso do phantom). Os resultados mostraram que o método é capaz de produzir campos de velocidade compatíveis aos medidos por contraste de fase e que ao mesmo tempo satisfazem as equações da mecânica dos fluidos. Mostrou-se que a 
técnica combinada pode ser utilizada para reduzir o ruído das medidas por contraste de fase, e considerando o campo de velocidades medido por contraste de fase como "ground truth", os resultados são mais realistas (qualitativamente e quantitativamente) que os obtidos com simulações puramente computacionais do escoamento sanguíneo para o modelo numérico utilizado. Foram ainda comparadas abordagens utilizando uma ou três componentes de velocidade medidas por RM para guiar as simulação de DFC.

Em seguida, foi proposto um método que torna possível a extração de mapas de velocidade de alta resolução espacial a partir de distribuições de velocidade medidas com codificação de velocidade em Fourier, com baixa resolução espacial. Baseado em distribuições CVF simuladas a partir dos dados de contraste de fase medidos de um phantom, o modelo do sinal das distribuições CVF foi deconvoluído, produzindo uma aproximação da distribuição CVF de alta resolução espacial, da qual é extraído o mapa de velocidades através de um processo de minimização proposto neste trabalho. Os resultados mostraram que o método proposto leva à obtenção de mapas de velocidade, no formato análogo aos do contraste de fase, razoavelmente precisos, a partir das distribuições de velocidade adquiridas com CVF.

Finalmente, foi demonstrada a possibilidade de utilizar as distribuições de velocidade com codificação em Fourier para guiar simulações computacionais do escoamento sanguíneo, utilizando mapas de velocidade extraídos das distribuições adquiridas com codificação de velocidade em Fourier. As distribuições CVF que foram simuladas a partir dos dados de contraste de fase do phantom foram utilizadas como base para a extração de campos de velocidade, utilizando o método proposto, mencionada acima. Assim, mostramos que as soluções guiadas por apenas uma componente de velocidade extraída da distribuição CVF está mais próxima dos dados de contraste de fase que a de uma simulação computacional pura, i.e., com influência mínima dos dados de ressonância magnética, para a modelagem utilizada neste trabalho. Além disso, houve um ligeiro ganho na relação sinal-erro para as componentes de velocidade extraídas das distribuições com CVF após a simulação computacional.

Importante ressaltar que os dados de contraste de fase para o phantom de escoamento pulsátil, utilizados neste trabalho, foram adquiridos em estágio de doutorado pelo programa Ciências sem Fronteiras (coordenado pela CAPES/CNPq) em um período de três meses (entre novembro/13 a janeiro/14) na University of Michigan, localizada na cidade de Ann 
Arbor, EUA. O estágio foi realizado no Laboratório de Ressonância Magnética Funcional sob a supervisão do Pesquisador Jon-Friedrik Nilsen. O laboratório possuia infraestrutura adequada para a coleta dos dados, tendo duas máquinas de ressonância magnética dedicadas apenas a pesquisa. Neste período, foi adquirido um conjunto de dados de velocidade obtidos com contraste de fase 4D Cartesiano de alta resolução espacial e alta relação sinal-ruído. A importância desses dados para a continuidade deste trabalho é de valor inestimável.

Os resultados aqui obtidos condizem com o que foi proposto no plano de trabalho. Esse trabalho até o momento resultou em várias publicações, a saber: um capítulo de livro internacional; três artigos em conferências internacionais especializadas; e um artigo em conferência nacional. Além disso, foram submetidos para publicação um artigo na revista Magnetic Resonance in Medicine (QUALIS A2); um artigo para a próxima conferência internacional da "Sociedade Internacional para Ressonância Magnética em Medicina" (ISMRM) e, ainda, um artigo para a próxima conferência internacional do IEEE "International Symposium on Biomedical Imaging". Finalmente, existe a expectativa de submissão de mais um trabalho para uma revista especializada, levando em consideração os resultados obtidos com o método combinado CVF-DFC.

Por fim, ainda existem diversas análises a respeito dos métodos aqui apresentados que não foram contempladas nesta tese, o que abre a possibilidade de um vasto campo de pesquisa e trabalhos na área de integração entre as técnicas do imageamento de fluxo na ressonância magnética e também as simulações da dinâmica dos fluidos computacional. Algumas questões que não puderam ser atacadas nessa tese e que são de interesse para trabalhos futuros são sugeridas a seguir:

1. Realizar a montagem de um experimento, utilizando um sistema de medição de velocidade por anemômetro laser Doppler [85], para medir de forma confiável e precisa as velocidades no interior do phantom. Essas medidas servirão de referência para a aquisição do campo de velocidades padrão, de modo a validar, com maior precisão, o método proposto, nesta tese, que permite a correção dos campos de velocidade medidos por RM utilizando as simulações numéricas através da dinâmica dos fluidos computacional;

2. Aplicar o método que combina dados de ressonância magnética e a dinâmica dos fluidos computacionais para distribuições reais adquiridas com codificação de velocidade em 
Fourier. A validação do método de recuperação de mapas de velocidade utilizando dados reais é importante, pois os mesmos são adquiridos de forma mais rápida que os dados por contraste de fase e com maior relação sinal-ruído;

3. Testar algumas possiblidades para reduzir o tempo de exame: (i) adquirir os dados de RM com resolução espacial reduzida e usar o método proposto para obter campos de velocidade com melhor resolução espacial; (ii) adquirir porções do volume (por exemplo, a entrada e a saída da carótida) com resolução espacial fina, enquanto que nas demais partes do volume os dados são adquiridos com resolução espacial menor; (iii) adquirir apenas alguns poucos cortes em volta da bifurcação e utilizar as simulações numéricas para preencher os espaços; (iv) medir uma ou duas componentes de velocidade com resolução espacial fina, enquanto que a(s) outra(s) é/são medida(s) com uma resolução espacial mais grosseira; (v) medir uma ou duas componentes de velocidade em todo o volume, enquanto que a(s) outra(s) componente(s) é(são) medida(s) somente em alguns cortes;

4. Implementar, através do software livre freeFEM ++ [86], o método combinado ressonância magnética e dinâmica dos fluidos computacional. O supracitado software permite soluções computacionais em malhas mais gerais, utilizando o método dos elementos finitos. No caso dos elementos finitos as equações de Navier-Stokes são também linearizadas e um problema linear semelhante ao mostrado neste trabalho é resolvido. O freeFEM ++ permite a manipulação das matrizes $\mathbf{A}_{\omega}$ e dos vetores $\mathbf{b}_{\omega}$ $(\omega=u ; v$; ou $w)$, que formam a base dos sistemas lineares que serão modificados para incluir os dados da ressonância magnética, como descrito no Capítulo 4. Tendo em vista a generalidade do método dos elementos finitos, poderemos obter parâmetros que não foram possíveis neste trabalho, como, por exemplo, a tensão de cisalhamento na parede, observando que será possível obter malhas suficientemente refinadas na parede dos vasos, além disso também será possível trabalhar em domínios com paredes elásticas. Através do freeFEM ++ também poderemos incluir os efeitos não-Newtonianos do sangue, os quais não foram analisados neste trabalho. 


\section{Referências Bibliográficas}

[1] MANSUR, A. P.; FAVARATO, D. Mortalidade por doenças cardiovasculares no Brasil e na região metropolitana de São Paulo: Atualização 2011. Arquivos Brasileiros de Cardiologia, v. 99, n. 2, p. $755-761,2012$.

[2] GOMES, R. Doenças cardiovasculares causam quase 30\% das mortes no país. 2011. Disponível em: <http://www.brasil.gov.br/sobre/saude/saude-do-homem/doencas-cardiovasculares >.

[3] Manning, W. J.; PEnNell, D. J. Cardiovascular Magnetic Resonance. 2nd. ed. Philadelphia, PA, USA: Saunders/Elsevier, 2010.

[4] O'DONNELL, M. NMR blood flow imaging using multiecho, phase contrast sequences. Medical Physics, v. 12(1), p. 59-64, 1985.

[5] PELC, N. et al. Phase-contrast cine magnetic resonance imaging. Magnetic Resonance Quarterly, v. 7, p. 229-254, 1991.

[6] PELC, N. et al. Quantitative magnetic resonance flow imaging. Magnetic Resonance Quarterly, v. 10, p. 125-147, 1994.

[7] CLARKE, G. D. et al. Velocity-encoded, phase-difference cine MRI measurements of coronary artery flow: dependence of flow accuracy on the number of cine frames. Journal of Magnetic Resonance Imaging, v. 6, n. 5, p. 733-742, 1996.

[8] MOHIADDIN, R. H. et al. Cine MR fourier velocimetry of blood flow through cardiac valves: comparison with doppler echocardiography. Journal of Magnetic Resonance Imaging, v. 7, n. 4, p. 657-663, 1997.

[9] MAN, L. et al. Nonsubtractive spiral phase contrast velocity imaging. Magnetic Resonance in Medicine, v. 42, p. 704-713, 1999. 
[10] NAYAK, K. S. et al. Real-time color flow MRI. Magnetic Resonance in Medicine, v. 43, p. $251-258,200$.

[11] MARKL, M. et al. Time-resolved three-dimensional phase-contrast MRI. Journal of Magnetic Resonance Imaging, v. 17, p. 499-506, 2003.

[12] LIU, C. Y. et al. Real-time color overlay cardiac phase contrast spiral imaging at 3 Tesla. In: SCMR Ninth Annual Scientific Sessions. Miami, FL, USA: The Society for Cardiovascular Magnetic Resonance, 2006.

[13] HARLOFF, A. et al. 3D blood flow characteristics in the carotid artery bifurcation assessed by flow-sensitive 4D MRI at 3T. Magnetic Resonance in Medicine, v. 61, p. 65-74, 2009.

[14] GONZALEZ, E.; CARVALHO, J. Does phase contrast MRI provide the mean velocity of the spins within a voxel? In: Proc. Intl. Soc. Mag. Reson. Med. 22. Milan, IT: International Society for Magnetic Resonance in Medicine, 2014. p. 2480.

[15] TANG, C.; BLATTER, D. D.; PARKER, D. L. Accuracy of phase-contrast flow measurements in the presence of partial-volume effects. Journal of Magnetic Resonance Imaging, v. 3(2), p. 377-385, 1993.

[16] MORAN, P. R. A flow velocity zeugmatographic interlace for NMR imaging in humans. Magnetic Resonance Imaging, v. 1, p. 197-203, 1982.

[17] DUMOULIN, C. L. et al. Quantitative measurement of blood flow using cylindrically localized Fourier velocity encoding. Magnetic Resononance in Medicine, v. 21, n. 2, p. 242-250, 1991.

[18] HENNIG, J. et al. Fast and exact flow measurements with the fast Fourier flow technique. Magnetic Resonance in Medicine, v. 6, n. 4, p. 369-372, 1988.

[19] GAlEA, D.; LAUZON, M. L.; DRANGOVA, M. Peak velocity determination using fast Fourier velocity encoding with minimal spatial encoding. Medical Physics, v. 29, n. 8, p. 1719$1728,2002$.

[20] MACGOWAN, C. K. et al. Real-time Fourier velocity encoding: an in vivo evaluation. Journal of Magnetic Resonance Imaging, v. 21, n. 3, p. 297-304, 2005.

[21] DICARLO, J. C. et al. Variable-density one-shot Fourier velocity encoding. Magnetic Resononance in Medicine, v. 54, n. 3, p. 645-655, 2005. 
[22] CARVAlHO, J. L. A. et al. Reconstruction of variable-density data in Fourier velocity encoding. In: Proc. Intl. Soc. Mag. Reson. Med. 15. Berlin, GER: International Society for Magnetic Resonance in Medicine, 2007. p. 2514.

[23] CARVALHO, J. L. A.; NAYAK, K. S. Rapid quantitation of cardiovascular flow using sliceselective Fourier velocity encoding with spiral readouts. Magnetic Resonance in Medicine, v. 57, p. 639-646, 2007.

[24] CARVAlHO, J. L. A. Velocity-Encoded Magnetic Resonance Imaging: Acquisition, Reconstruction and Applications. Tese (Doutorado) — Department of Electrical Engineering, University of Southern California, August 2008.

[25] CARVAlho, J. L. A.; NIELSEN, J. F.; NAYAK, K. S. Feasibility of In Vivo measurement of carotid wall shear rate using spiral Fourier velocity encoded MRI. Magnetic Resonance in Medicine, v. 63, p. 1537-1547, 2010.

[26] TSUJI, T. et al. Vector analysis of the wall shear rate at the human aortoiliac bifurcation using cine MR velocity mapping. American Journal of Roentgenology, v. 178, p. 995-999, 2002.

[27] EBBERS, T. et al. Non-invasive measurement of time-varying three-dimensional relative pressure fields within the human heart. Journal of Biomechanical Engineering, v. 124, p. 288$293,2002$.

[28] STALDER, A. et al. Quantitative 2D and 3D phase contrast MRI: optimized analysis of blood flow and vessel wall parameters. Magnetic Resonance in Medicine, v. 60, p. 1218-1231, 2008.

[29] GIDDENS, D.; ZARINS, C.; GLAGOV, S. The role of fluid mechanics in the localization and detection of atherosclerosis. Journal of Biomechanical Engineering, v. 115, p. 588-594, 1993.

[30] LEI, M.; KLEINSTREUER, C.; TRUSKEY, G. Numerical investigation and prediction of atherogenic sites in branching arteries. Journal of Biomechanical Engineering, v. 117, n. 350$357,1995$.

[31] MILNER, J. et al. Hemodynamics of human carotid artery bifurcations: Computational studies with models reconstructed from magnetic resonance imaging of normal subjects. Journal of Vascular Surgery, v. 28, p. 143-156, 1998.

[32] LONG, Q. et al. Reconstruction of blood flow patterns in a human carotid bifurcation: a combined CFD and MRI study. Journal of Magnetic Resonance Imaging, v. 11, p. 299-311, 2000 . 
[33] HYUN, S.; KLEINSTREUER, C.; ARCHIE, J. Hemodynamics analyses of arterial expansions with implications to thrombosis and restenosis. Medical Engineering Physics, v. 22, p. 13-27, 2000.

[34] PAPATHANASOPOULOU, P. et al. MRI measurement of time-resolved wall shear stress vectors in a carotid bifurcation model, and comparison with CFD predictions. Journal of Magnetic Resonance Imaging, v. 17, p. 153-162, 2003.

[35] LONGEST, P.; KLEINSTREUER, C. Comparison of blood particle deposition models for non-parallel flow domains. Journal of Biomechanics, v. 36, p. 421-430, 2003.

[36] THOMAS, J. et al. Reproducibility of image-based computational fluid dynamics models of the human carotid bifurcation. Annals of Biomedical Engineering, v. 31, p. 132-141, 2003.

[37] STEINMAN, D. Image-based computational fluid dynamics: A new paradigm for monitoring hemodynamics and atherosclerosis. Current Drug Targets - Cardiovascular and Hematological Disorders, v. 4, p. 183-197, 2004.

[38] MARSHALL, I. et al. MRI and CFD studies of pulsatile flow in healthy and stenosed carotid bifurcation models. Journal of Biomechanics, v. 37, p. 679-687, 2004.

[39] STEINMAN, D.; TAYLOR, C. Flow imaging and computing: Large artery hemodynamics. Annals of Biomedical Engineering, v. 33, p. 1704-1709., 2005.

[40] CANSTEIN, C. et al. 3D MR flow analysis in realistic rapid-prototyping model systems of the thoracic aorta: comparison with in vivo data and computational fluid dynamics in identical vessel geometries. Magnetic Resonance in Medicine, v. 59, n. 3, p. 535-546, 2008.

[41] BOUSSEL, L. et al. Phase-contrast magnetic resonance imaging measurements in intracranial aneurysms in vivo of flow patterns, velocity fields, and wall shear stress: comparison with computational fluid dynamics. Magnetic Resonance in Medicine, v. 61, n. 2, p. 409-417, 2009.

[42] BUONOCORE, M. Algorithms for improving calculated streamlines in 3-D phase contrast angiography. Magnetic Resonance in Medicine, v. 31, n. 1, p. 22-30, 1994.

[43] SONG, S. M. et al. Noise reduction in three-dimensional phase contrast MR velocity measurements. Journal of Magnetic Resonance Imaging, v. 3, n. 587-596, 1993.

[44] BUSCH, J. et al. Reconstruction of divergence-free velocity fields from cine 3d phase-contrast flow measurements. Magnetic Resonance in Medicine, v. 1, n. 69, p. 200-210, 2013. 
[45] VERDUGO, A. M. P.; MURA, J.; URIBE, S. Enforcing divergence free to velocity data from 4D flow MR images. In: Proc. Intl. Soc. Mag. Reson. Med. 22. Milan, IT: International Society for Magnetic Resonance in Medicine, 2014. p. 2485.

[46] LIU, L.; FUNAMOTO, K.; HAYASE, T. Numerical experiment for ultrasonic-measurementintegrated simulation of developed laminar pipe flow using axisymmetric model. Journal of Biomechanical Science and Engineering, v. 3, p. 101-115, 2008.

[47] PATANKAR, S. V. Numerical Heat Transfer and Fluid Flow. 1st. ed. New York, NY, USA: Hemisphere Publishing Corporation, 1980.

[48] NIELSEN, J.-F.; NAYAK, K. S. MR-driven computational fluid dynamics. In: Proc. Intl. Soc. Mag. Reson. Med. 17. Honolulu, HI, USA: International Society for Magnetic Resonance in Medicine, 2009. p. 3858.

[49] RISPOLI, V. C. et al. Assessment of carotid flow using magnetic resonance imaging and computational fluid dynamics (chapter 23). In: Fluid Dynamics, Computational Modeling and Applications. Rijeka, HR: Intech, 2012. p. 513-536.

[50] RISPOLI, V. C. et al. Computational fluid dynamics simulations guided by 3D PC-MRI data. In: Proc. Intl. Soc. Mag. Reson. Med. 22. Milan, IT: International Society for Magnetic Resonance in Medicine, 2014. p. 2490.

[51] RISPOLI, V. C. et al. Simulações computacionais do escoamento sanguíneo guiadas por dados de contraste de fase. In: Anais do XXIV Congresso Brasileiro de Engenharia Biomédica. Uberlândia, MG: Sociedade Brasileira de Engenharia Biomédica, 2014. p. 1066-1069.

[52] RISPOLI, V. C.; CARVALHO, J. Deriving high-resolution velocity maps from low-resolution Fourier velocity encoded MRI data. In: IEEE 10th International Symposium on Biomedical Imaging. San Francisco, CA, USA: Institute of Electrical and Electronics Engineers, 2013. p. 334-337.

[53] RISPOLI, V.; CARVALHO, J. High-resolution, high-SNR velocity maps reconstructed from low-resolution Fourier velocity encoded MRI data. In: Proc. Intl. Soc. Mag. Reson. Med. 21. Salt Lake City, UT, USA: International Society for Magnetic Resonance in Medicine, 2013. p. 68.

[54] STEINMAN, D. A. et al. Reconstruction of carotid bifurcation hemodynamics and wall thickness using computational fluid dynamics and MRI. Magnetic Resonance in Medicine, v. 47, n. 1, p. 149-159, 2002. 
[55] PATANKAR, S.; SPALDING, D. A calculation procedure for heat, mass and momentum transfer in three-dimensional parabolic flows. International Journal of Heat and Mass Transfer, v. 15, p. $1787-1806,1972$.

[56] MAZZOLA, A. A. Ressonância magnética: princípios de formação da imagem e aplicações em imagem funcional. Revista Brasileira de Física Médica, v. 3, n. 1, p. 117-129, 2009.

[57] HU, B. S.; PAULY, J. M.; MACOVSKI, A. Localized real-time velocity spectra determination. Magnetic Resonance in Medicine, v. 30, n. 3, p. 393-398, 1993.

[58] NISHIMURA, D. Principles of Magnetic Resonance Imaging. 1st. ed. Stanford, CA, USA: Stanford University Press, 2010.

[59] SINGER, J. R. Blood flow rates by nuclear magnetic resonance measurements. Science, v. 130, n. 3389 , p. $1652-1653,1959$.

[60] HAHN, E. L. Detection of sea-water motion by nuclear precession. Journal of Geophysical Research, v. 65, n. 2, p. 776-777, 1960.

[61] SINGER, J. R.; CROOKS, L. E. Nuclear magnetic resonance blood flow measurements in the human brain. Science, v. 221, n. 4611, p. 654-656, 1983.

[62] DIJK, P. van. Direct cardiac NMR imaging of heart wall and blood flow velocity. Journal of Computer Assisted Tomography, v. 8, n. 3, p. 429-436, 1984.

[63] REBERGEN, S. A. et al. Magnetic resonance measurement of velocity and flow: technique, validation, and cardiovascular applications. American Heart Journal, v. 126, n. 6, p. 1439-1456, 1993.

[64] BIRD, R.; ARMSTRONG, R.; HASSAGER, O. Dynamics Of Polymeric Liquids: Fluid Mechanics. 2nd. ed. New York, NY, USA: Willey, 1987.

[65] KIM, S. A Study of Non-Newtonian Viscosity and Yield Stress of Blood in a Scanning Capillary-Tube Rheometer. Tese (Doutorado) - Drexel University, 2002.

[66] KIM, S. et al. Determination of rheological properties of whole blood with a scanning capillarytube rheometer using constitutive models. Journal of Mechanical Science and Technology, v. 23, n. 6 , p. $1728-1726,2009$.

[67] PERKTOLD, K.; RAPPITSCH, G. Computer simulation of local blood flow and vessel mechanics in a compliant carotid artery bifurcation model. Journal of Biomechanics, v. 28, n. 7, p. 845-856, 1995. 
[68] FIGUEROA, C. A. et al. A coupled momentum method for modeling blood flow in threedimensional deformable arteries. Computer Methods in Applied Mechanics and Engineering, v. 195, p. $5685-5706,2006$.

[69] CHORIN, A.; MARSDEN, J. A Mathematical Introduction to Fluid Mechanics. 3rd. ed. New York, NY, USA: Springer, 2000.

[70] VERSTEEG, H.; MALALASEKERA, W. An Introduction to Computational Fluid Dynamics: The Finite Volume Method. 2nd. ed. Glasgow, SCT, UK: Prentice-Hall, 2007.

[71] HARLOW, F. H.; WELCH, J. E. Numerical calculation of time-dependent viscous incompressible flow of fluid with free surface. The Physics of Fluids, v. 8, n. 12, p. 2182-2189, 1965.

[72] GHIA, U.; GHIA, K.; SHIN, C. High-Re solutions for incompressible flow using the Navier-Stokes equations and a multigrid method. Journal of Computational Physics, v. 48, p. $387-411,1982$.

[73] GUPTA, M.; KALITA, J. A new paradigm for solving Navier-Stokes equations: streamfunction-velocity formulation. Journal of Computational Physics, v. 207, p. 52-68, 2005.

[74] MACHADO, M. R. Elaboração e Validação de um Fluido Mimetizador do Sangue Humano Quanto às Suas Propriedades Ultra-Sônicas, Reológica e Térmica. Dissertação (Mestrado) COPPE / UFRJ, 2009.

[75] PLANTE, E.; TURKSTRA, L. Sources of error in the quantitative analysis of MRI scans. Magnetic Resonance Imaging, v. 9, n. 4, p. 589-595, 1991.

[76] Bernstein, M.; KING, K. F.; ZHOU, X. J. Handbook of MRI Pulse Sequences. 1st. ed. San Diego, CA, USA: Elsevier, 2004.

[77] LINGAMNENI, A. et al. Validation of cine phase-contrast MR imaging for motion analysis. Journal of Magnetic Resonance Imaging, v. 5, p. 331-338, 1995.

[78] WeSterhof, N.; STERGIOPUlOS, N.; NOBLE, M. Snapshots of Hemodynamics: An Aid for Clinical Research and Graduate Education. 1st. ed. New York, NY, USA: Springer, 2005.

[79] HANSEn, P. C.; NAGY, J. G.; O'LEARY, D. P. Deblurring Images. Matrices, Spectra and Filtering. Philadelphia, PA, USA: Society for Industrial and Applied Mathematics, 2006. (Fundamentals of Algorithms). 
[80] VELHO, H. F. C. Problemas inversos: Conceitos básicos e aplicações - mini-curso. In: IV Encontro de Modelagem Computacional. Nova Friburgo, RJ: IPRJ-UERJ, 2001. p. 1-17.

[81] BERTERO, M.; BOCCACCI, P. Image deconvolution (chapter 8). In: From Cells to Proteins: Imaging Nature across Dimensions. Pisa, IT: Springer, 2005. p. 349-370.

[82] KRISHNAN, D.; FERGUS, R. Fast image deconvolution using hyper-Laplacian priors. In: Advances in Neural Information Processing Systems 22. Vancouver, CA: Curran Associates, Inc., 2009. p. 1033-1041.

[83] ROTH, S.; BLACK, M. J. Fields of experts: A framework for learning image priors. In: Proceedings of IEEE Computer Society Conference on Computer Vision and Pattern Recognition. San Diego, CA, USA: Institute of Electrical and Electronics Engineers, 2005. v. 2, p. 860-867.

[84] WANG, Y. et al. A new alternating minimization algorithm for total variation image reconstruction. SIAM Journal on Imaging Sciences, v. 1, n. 3, p. 248-272, 2008.

[85] DURST, F. Principles and Practices of Laser-Doppler Anemometry. 1st. ed. Waltham, MA, USA: Academic Press Inc., 1981.

[86] HECHT, F. New development in freefem++. Journal of Numerical Mathematics, v. 20, n. 3-4, p. 251-265, 2012. 
ANEXOS 


\section{PUBLICAÇÕES}

Utilizando os métodos e a modelagem física propostas, temos que as contribuições relacionadas a este trabalho de doutorado até o momento são:

\section{Periódico}

1. Rispoli, V.C., Carvalho, J.L.A., Nielsen, J-F., Nayak, K. S. Blood flow computational fluid dynamics simulations influenced by 3D PC-MRI data. Em: Magnetic Resonance in Medicine, 2014. (Submetido)

2. Rispoli, V.C., Carvalho, J.L.A., Nielsen, J-F., Nayak, K. S. FVE-driven computational fluid dynamics blood flow simulations. (Em preparação)

\section{Capítulo de livro}

1. Rispoli, V.C., Carvalho, J.L.A., Nielsen, J-F., Nayak, K. S. Assessment of Carotid Flow Using Magnetic Resonance Imaging and Computational Fluid Dynamics. Em: L. Hector Juarez. (Org.). Fluid Dynamics, Computational Modeling and Applications. 1ed.: InTech, 2012, v. 3, p. 513-536. 49]

\section{Conferência internacional}

1. Rispoli, V.C., Nilsen, J-F., Nayak, K.S., Carvalho, J.L.A. Computational fluid dynamics simulations guided by Fourier velocity encoded MRI. Em: Proc. Intl. Soc. Mag. Reson. Med. 23, Toronto, Canadá, 2015. (Submetido)

2. Rispoli, V.C., Nilsen, J-F., Nayak, K.S., Carvalho, J.L.A. Using Fourier velocity encoded MRI data to guide CFD simulations. Em: International Symposium on Biomedical Imaging, Nova York, EUA, 2015. (Submetido)

3. Rispoli, V.C., Nilsen, J-F., Nayak, K.S., Carvalho, J.L.A. Computational fluid dynamics simulations guided by $3 D$ PC-MRI data. Em: Proc. Intl. Soc. Mag. Reson. Med. 22, Milão, Itália, 2014, p. 2490. [50]

4. Rispoli, V.C., Carvalho, J.L.A. Deriving high-resolution velocity maps from low-resolution Fourier velocity encoded MRI data. Em: 2013 IEEE 10th International Symposium on Biomedical Imaging, San Francisco, EUA, 2013, p. 334-337. [52] 
5. Rispoli, V.C., Carvalho, J.L.A. High-resolution, high-SNR velocity maps reconstructed from low-resolution Fourier velocity encoded MRI data. Em: Proc. Intl. Soc. Mag. Reson. Med. 21, Salt Lake City, EUA, 2013, p. 68. [53]

\section{Conferência nacional}

1. Rispoli, V.C., Carvalho, J.L.A., Nielsen, J-F., Nayak, K. S. Simulações Computacionais do Escoamento Sanguíneo Guiadas por Dados de Contraste de Fase. Em: XXIV Congresso Brasileiro de Engenharia Biomédica, Uberlândia, Brasil, 2014, p. 1066-1069. [51] 